\title{
EXTENSION-TWIST COUPLING OPTIMIZATION IN COMPOSITE ROTOR BLADES
}

\author{
A Ph.D. Thesis \\ Presented to the Academic Faculty \\ by \\ Serkan Ozbay \\ In Partial Fulfillment \\ of the Requirements for the Degree of \\ Doctor of Philosophy \\ in the School of Aerospace Engineering
}

Georgia Institute of Technology

May 2006 
To my Parents Nedime \& Engin Ozbay and My Lovely Wife Tuba 


\section{EXTENSION-TWIST COUPLING OPTIMIZATION IN COMPOSITE ROTOR BLADES}

Approved by:

Prof. Erian A. Armanios, Advisor Dr. D. Stefan Dancila School of Aerospace Engineering Georgia Institute of Technology

Dancila LLC

Prof. Olivier A. Bauchau

School of Aerospace Engineering Georgia Institute of Technology

Prof. Abdul Hamid Zureick

School of Civil \& Environmental Engineering Georgia Institute of Technology

Prof. Dewey H. Hodges

School of Aerospace Engineering Georgia Institute of Technology

Date Approved : December 12, 2005 


\section{Acknowledgments}

This work could not have been completed without the help of many people to whom I would like to give my thanks.

I would like to express my deepest gratitude to Dr. Erian Armanios for his patient guidance throughout my life at Georgia Tech. This work would have never been possible without his immeasurable professional and emotional support, encouragement and above all his trust.

I am also grateful to Dr. D. Stefan Dancila for his support in this work. His valuable critiques and guidance helped me improve my work. I also would like to acknowledge Dr. Olivier A. Bauchau. I am thankful to him for providing the DYMORE and VABS programs. I would like to thank Dr. Dewey H. Hodges and Dr. Abdul-Hamid Zureick for their willingness to serve on my thesis committee.

This work has been completed under a grant from the National Rotorcraft Technology Center. I am sincerely thankful for their support.

Additionally I would like to thank my officemates, true friends, Samer Tawfik, Xinyuan Tan and Dr. Roxana Vasilescu for their technical and emotional support.

I sincerely thank my parents Nedime and Engin Ozbay. Without their love, support and sacrifices, I would not be who I am now. Thank you for your belief in what I could achieve eventually.

Finally I would like to express my great gratitude to my lovely wife Tuba, for her unconditional love, support and confidence. It is a huge understatement to say that I could achieve this without her. I love her. The rest of my days are special because of her. 


\section{TABLE OF CONTENTS}

Acknowledgments ..................................................................................................... iv

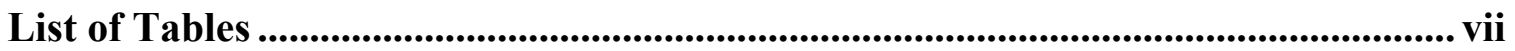

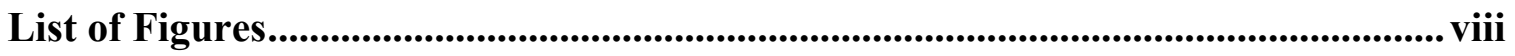

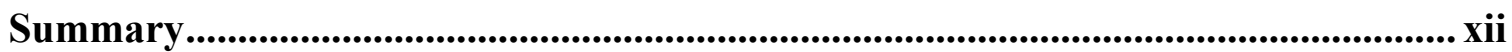

CHAPTER 1 Introduction ..................................................................................... 1

CHAPTER 2 A Survey of Passive Blade Twist Control Applications ........................ 5

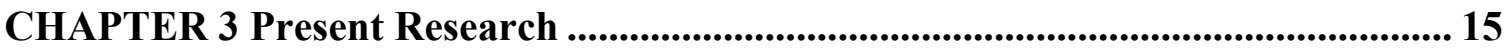

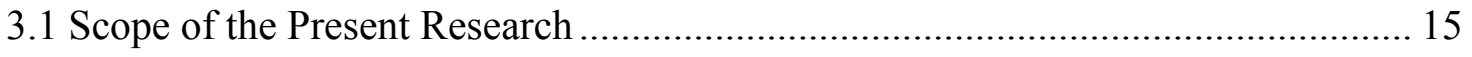

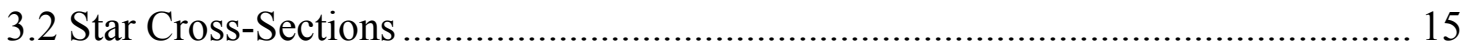

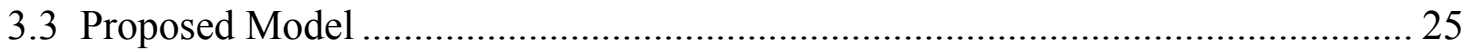

3.3.1 Rotor Blade Cross-Sectional Analysis ....................................................... 30

3.3.2 Multibody Dynamics Modeling for Rotorcraft Applications ......................... 31

3.3.3 Tiltrotor Aircraft Optimization Studies................................................. 33

CHAPTER 4 Modeling of the Proposed Design........................................................... 37

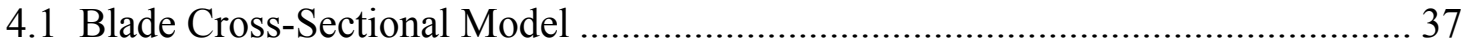

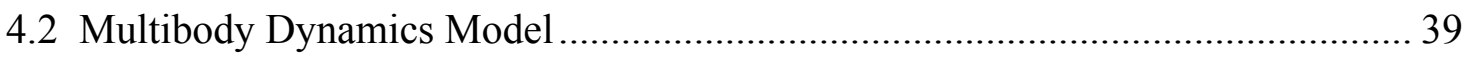

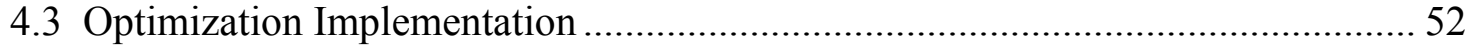

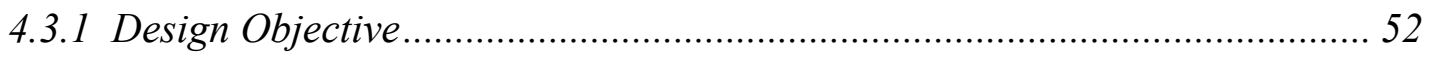

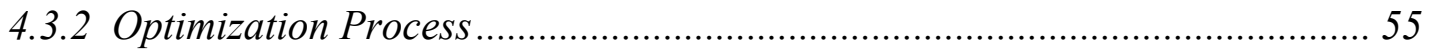




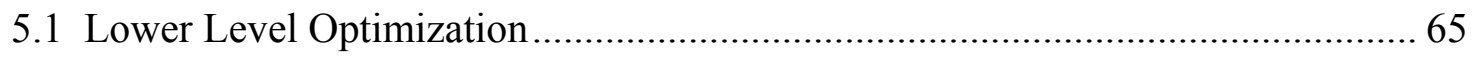

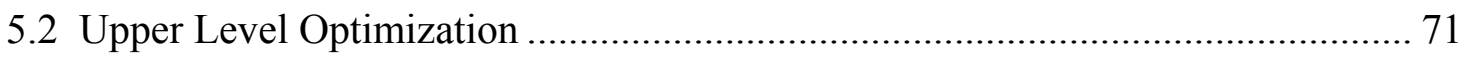

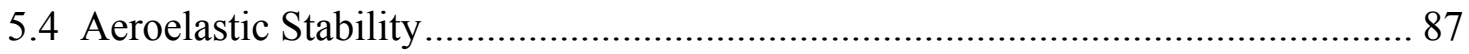

CHAPTER 6 Conclusion and Recommendations .......................................................93

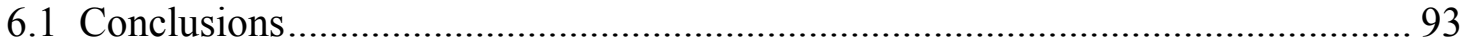

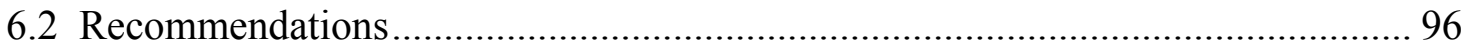

Appendix ............................................................................................................................ 98

A. Spanwise Variable Layup Case........................................................................ 98

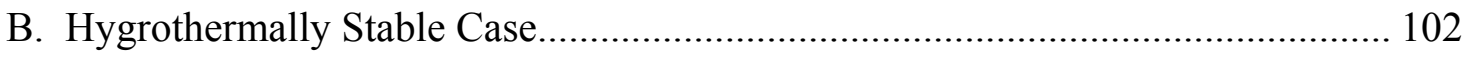

References ....................................................................................................................................... 105 


\section{List of Tables}

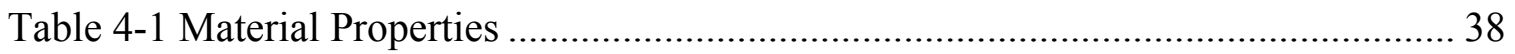

Table 4-2 Baseline System Properties ................................................................. 40

Table 4-3 Non-rotating Collective Mode Blade Frequencies ....................................... 49

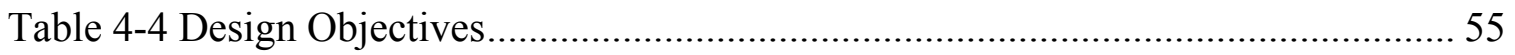

Table 5-1 Optimum Lower-Level Design Parameters ................................................ 66

Table 5-2 Upper-Level Optimum Design Parameters ............................................... 72

Table 5-3 Optimized Model Modified Stacking Sequences ...................................... 78

Table 5-4 ABAQUS Model Geometric and Material Properties................................. 86

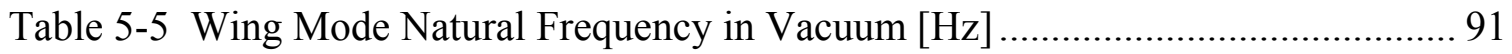

Table A-1 Optimum Design Parameters for Spanwise Variable Layup Case ................. 98

Table B-1Optimum Design Parameters ............................................................... 102 


\section{List of Figures}

Figure 1-1. Comparison of Blade Built-in Twist with Power Requirement ..................... 3

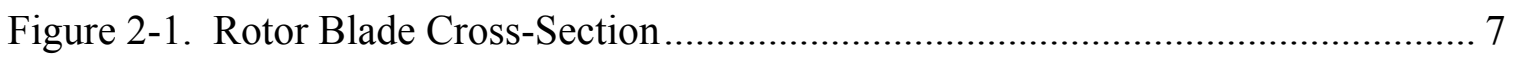

Figure 2-2. Composite Blade Cross-Section........................................................ 9

Figure 2-3. Extension-Twist Coupled Blade Twist Distributions with Varying Tip

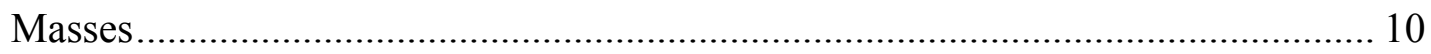

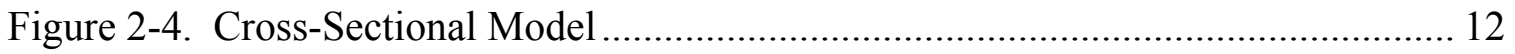

Figure 3-1. Extension-Twist Coupled Cross-Sections with Tailored Stiffness ............... 17

Figure 3-2. Star-Beam Cross-Sectional Configurations …...................................... 18

Figure 3-3. Modified Star Configurations with Cylindrical Central Core...................... 18

Figure 3-4. Comparison of Extension-Twist Coupling from Star and Modified Star

Configuration with Antisymmetric Layup................................................... 19

Figure 3-5. FE Model of an Eight-Finned Star Section with a Circular Core ................ 20

Figure 3-6. Torsional Stiffness vs. Extension-Twist Coupling of Star Configurations with Varying Number of Fins (n) and Core Radius ............................................... 21

Figure 3-7. Comparison of Normalized Torsional Stiffness and Extension-Twist

Coupling with respect to 4-Finned [ $\mathrm{n}=4$ ] Case ................................................ 22

Figure 3-8. Torsional Stiffness and Extension-Twist Coupling Constants..................... 24

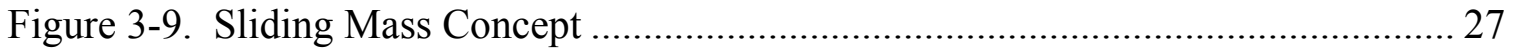

Figure 3-10. Blade Elastic Twist Comparisons ...................................................... 28

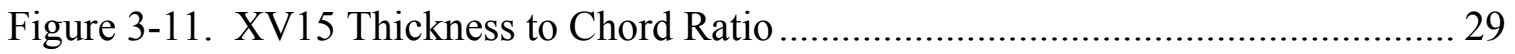

Figure 4-1. Blade VABS Cross-Sectional Model ................................................. 38

Figure 4-2. XV15 DYMORE Model in Hover Mode.................................................. 41 
Figure 4-3. XV15 DYMORE Model in Cruise Mode 41

Figure 4-4. Baseline Bending Stiffness Distributions.................................................... 42

Figure 4-5. Baseline Torsional Stiffness Distribution ................................................. 42

Figure 4-6. Baseline Mass and Thickness/Chord (t/c) Ratio Distribution....................... 43

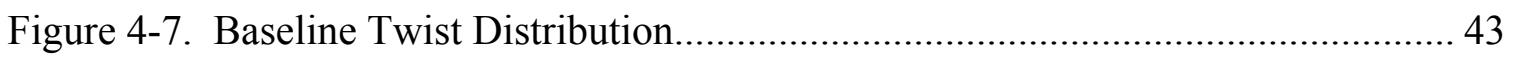

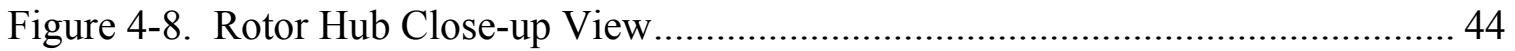

Figure 4-9. Baseline Power vs. Thrust Coefficient Comparison ..................................... 45

Figure 4-10. Baseline Thrust Coefficient vs. Collective Pitch at $R=0.75$ Comparison 46

Figure 4-11. DYMORE Model for Sliding Mass Concept .............................................. 47

Figure 4-12. Frequency vs. Rotor Speed ………………………………………...... 51

Figure 4-13. Twist Rate Distributions ……………………......................................... 53

Figure 4-14. Change in Power Requirement in Hover at $C_{T}=0.01$ vs. Twist Rate ........ 53

Figure 4-15. Change in Power Requirement in Cruise at $C_{T}=0.01$ vs. Twist Rate........ 54

Figure 4-16. Multilevel Optimization Process ............................................................... 56

Figure 4-17. Composite Box Beam Model .................................................................... 57

Figure 4-18. Lower Level Optimization DYMORE Model ............................................ 58

Figure 4-19. Upper Level Optimization Cross-sectional Model ..................................... 60

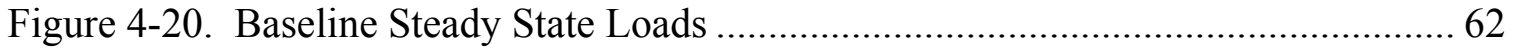

Figure 4-21. Blade Loading Coordinate System........................................................... 62

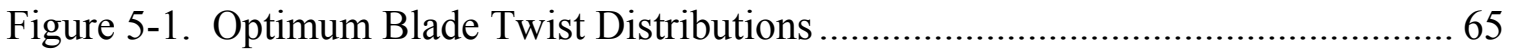

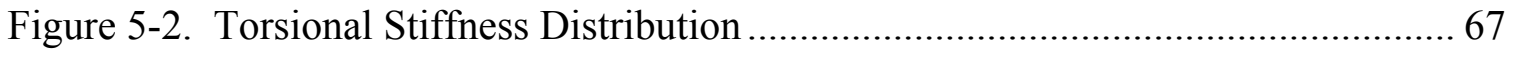

Figure 5-3. Optimum Extension-Twist Coupling Distribution........................................ 68

Figure 5-4. Blade Twist Comparison with the Target Values ......................................... 69 
Figure 5-5. Power vs. Thrust Coefficient for Lower-Level Optimized System 70

Figure 5-6. Cost Function vs. Number Generated in Simulated Annealing Based Optimizer 72

Figure 5-7. Extension-Twist Coupling Distribution ............................................... 74

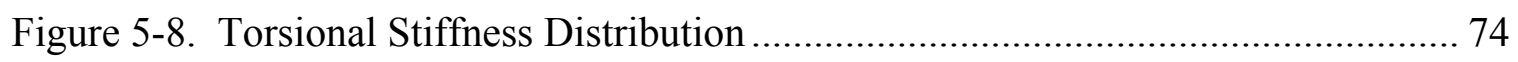

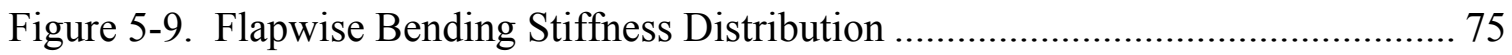

Figure 5-10. Chordwise Bending Stiffness Distribution ....................................... 75

Figure 5-11. Power vs. Thrust Coefficient of the Optimized System............................ 77

Figure 5-12. Single Layer of $\left[45^{\circ} /-45^{\circ}\right]$ Angle-ply Application on the Model ........... 78

Figure 5-13. Extension-Twist Coupling Distribution for the Modified System............. 79

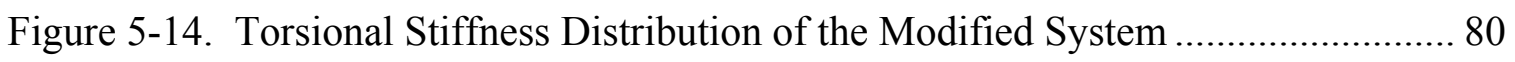

Figure 5-15. Flapwise Bending Stiffness Distribution of the Modified System ............ 81

Figure 5-16. Chordwise Bending Stiffness Distribution of the Modified System.......... 81

Figure 5-17. Untwisted Model Flapwise Bending Stiffness ...................................... 82

Figure 5-18. Untwisted Model Chordwise Bending Stiffness .................................. 83

Figure 5-19. Effect of the $\left[45^{\circ} /-45^{\circ}\right]$ Angle-ply Layer on the Final Twist Distributions

Figure 5-20. Power vs. Thrust Coefficient for the System with the $\left[45^{\circ} /-45^{\circ}\right]$ Angle-

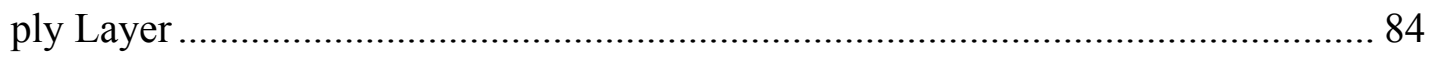

Figure 5-21. Representative ABAQUS Model ..................................................... 85

Figure 5-22. Twist Rate vs. Temperature Change of the Representative Model............ 87

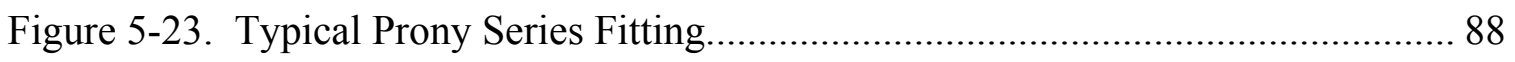

Figure 5-24. Isolated Rotor Frequency vs. Rotor Speed......................................... 90 


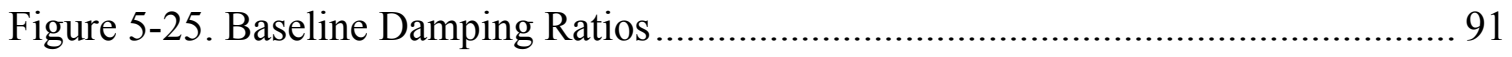

Figure 5-26. Optimized System Damping Ratios ..................................................... 92

Figure 5-27. Damping Ratio Comparison at 310 Knots ........................................... 92

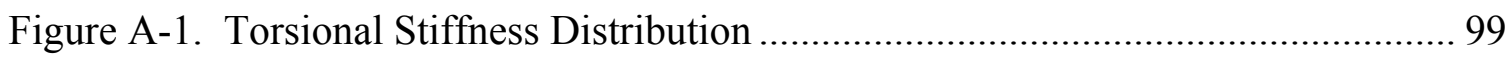

Figure A-2. Extension-Twist Coupling Distribution .............................................. 99

Figure A-3. Power vs. Thrust Coefficient Comparison ........................................... 101

Figure B-1. Torsional Stiffness Distribution .................................................... 103

Figure B-2. Extension-Twist Coupling Distribution ............................................ 103

Figure B-3. Power vs. Thrust Coefficient Comparison ........................................... 104 


\section{Summary}

For optimal rotor performance in a tiltrotor aircraft the difference in the inflow and the rotor speeds between the hover and cruise flight modes suggests different blade twist and chord distributions. The blade twist rates in current tiltrotor applications are defined based upon a compromise between the figure of merit in hover and propeller efficiency in airplane mode. However, when each operation mode is considered separately the optimum blade distributions are found to be considerably different.

Passive blade twist control (PBTC), which uses the inherent variation in centrifugal forces on a rotor blade to achieve optimum blade twist distributions in each flight mode through the use of extension-twist (ET) coupled composite rotor blades, has been considered for performance improvement of tiltrotor aircraft over the last two decades. The challenge for this concept is to achieve the desired twisting deformations in the rotor blade without altering the aeroelastic characteristics of the vehicle.

Extension-twist coupled composite structures with star cross-sections were

proposed as a novel way to provide adequate levels of twisting deformation for PBTC applications. In the present research, the feasibility analysis of such structures revealed a conflicting behavior between the ET coupling and the torsional stiffness. A concept referred to as the sliding mass concept is proposed in this work in order to increase the twist change with rotor speed for a closed-cell composite rotor blade cross-section to practical levels for performance improvement in a tiltrotor aircraft. The concept is based 
on load path changes for the centrifugal forces by utilizing non-structural masses readily available on a conventional blade, such as the leading edge balancing mass.

A multilevel optimization technique based on the simulated annealing method is applied to improve the performance of the XV15 tiltrotor aircraft. The lower level optimization is based on determining required extension-twist coupling distribution and the sliding mass value utilizing a simple composite box-beam model. A cross-sectional analysis tool, VABS together with a multibody dynamics code, DYMORE are integrated into the optimization process. At the upper level optimization the coupling values from the lower level optimization are used as target values for a detailed cross-sectional sizing process. The optimization included constraints on the geometry, strength, stiffness and manufacturability.

The optimization results revealed significant improvements in the power requirement in hover while preserving cruise efficiency. It is also shown that about $21 \%$ of the improvement is provided through the sliding mass concept pointing to the additional flexibility the concept provides for tailoring of the structure without any additional weight penalty on the system. Through aeroelastic stability analysis the optimized system is found to be stable and the effect of the sliding mass concept on the damping characteristics of the overall system is found to be minimal. 


\section{CHAPTER 1}

\section{Introduction}

A tiltrotor aircraft is a vertical and short take-off and landing (V/STOL), multipurpose aircraft with high-speed cruise performance. It can perform a wide range of V/STOL missions as effectively as a conventional helicopter, but at the same time it is capable of achieving the long-range high speed cruise efficiencies of a twin turbo propeller aircraft.

The development of the tiltrotor aircraft started in early 1950s and Bell XV-3 performed its first helicopter to aircraft conversion in 1958. Whirl flutter instability was first observed on this vehicle. In the early 1970's the XV15 tiltrotor research aircraft was developed by Bell demonstrating the feasibility of the concept and has been extensively used by NASA and the Army to gain unique insight into the aeroelastic behavior of such vehicles. The success of the XV15 led to the development of V22 Osprey. The versatility of this vehicle also opened up new horizons for the commercial aircraft industry. The world's first civilian tiltrotor Bell/Agusta's BA609 is expected to get certified in the year 2007.

The most interesting feature of a tiltrotor aircraft is its rotor blade positioning in two main flight modes, namely hover (helicopter) and cruise (airplane) modes. In the hover mode the wingtip mounted rotors are oriented with their tip-path plane vertical while in the cruise mode they are rotated such that the tip-path planes are horizontal. This necessitates a compromise design between traditional helicopter and aircraft systems. The hover mode is characterized by small inflow and high blade loading. The high blade loading is caused by the blades carrying the entire aircraft weight and the wing download which in some cases can 
be as high as 10 to $15 \%$ of the total rotor thrust. In the airplane mode there is a considerably higher inflow compared to hover and the blade loading is lower.

In terms of rotor speeds in each flight mode there is about 15 to $20 \%$ difference. In the airplane mode the XV15 operates at about $80 \%$ of the hover mode rotor speed. The rotor speed in hover is defined based on maximizing the thrust for a given power level. In the airplane mode, due to the compressibility effects at the blade tips, the rotor speed has to be reduced. In this mode the rotor speed can be chosen based on maximum air speed, maximum range or service ceiling.

The difference in the inflow and the rotor speeds between the two flight modes suggests different blade twist and chord distributions. The blade twist rates in the current tiltrotor applications are defined based on a compromise between the figure of merit in hover and propeller efficiency in airplane mode. However, when each operation mode is considered separately the optimum blade distributions are found to be considerably different.

Figure 1-1 shows the power required in hover and airplane modes as a function of linear blade twist [47]. The optimum blade twist for hover is $20 \mathrm{deg}$ while it is above $45 \mathrm{deg}$ for the airplane mode. The figure points to a $6 \%$ improvement in the power requirement in both hover and airplane modes with respect to a compromise design of 36 deg linear twist. How much of this $6 \%$ improvement can be obtained is a matter of how close the optimum blade twist distributions can be achieved.

Over the last two decades several designs have been proposed in order to improve the performance of the tiltrotor aircraft having a compromise design between hover and cruise modes. Variable diameter tiltrotor (VDTR) is an example of such an approach in which the rotor diameter is changed between hover and cruise modes providing near optimum 
conditions for each flight mode. Reference [22] provides an insight into the VDTR concept developed by Sikorsky Aircraft. The concept was found to be feasible and demonstrated to improve the overall performance of the aircraft. The approach eliminated the need to reduce the rotor speed in the cruise mode by decreasing the rotor diameter by up to $40 \%$ and thus reducing the blade tip speed and blade area considerably. The drawback of the system is the additional mechanism needed for the blade retraction and extraction between flight modes and the cost and weight penalty associated with it.

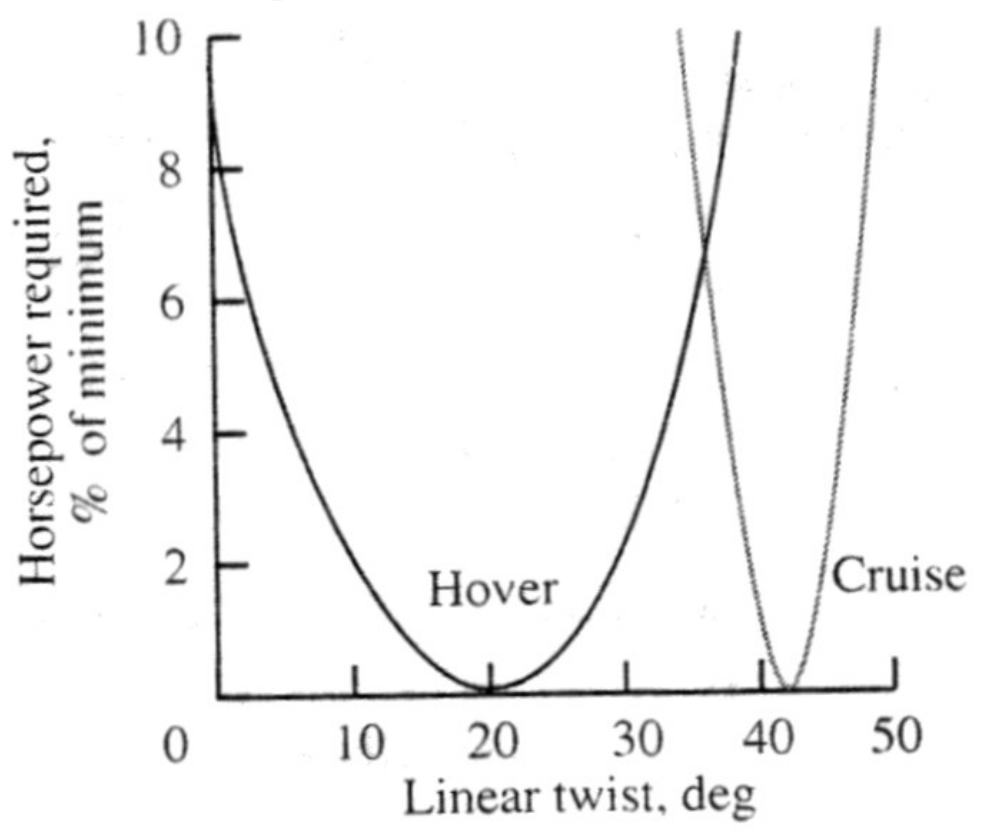

Figure 1-1. Comparison of Blade Built-in Twist with Power Requirement, [47] 
Smart materials have also been employed to alter twist distributions between two flight modes. Shape memory alloys (SMA) have been used as a bimoment actuator based upon warping-torsion coupling in a torque tube configuration [52]. Such systems provide promising results in generating twist changes. However the energy required to actuate them substantially reduce the associated improvement in power which is of the order of 5 to $6 \%$.

One other promising means of performance enhancement in tiltrotor aircraft is passive blade control. The concept makes use of the anisotropic nature of composite materials and utilizes elastically tailored composite rotor blades. Unlike the VDTR systems this concept relies upon the inherent change in rotor speed between hover and airplane modes resulting in a net difference in blade forces and utilizes elastically tailored composite rotor blades incorporating elastic couplings such as extension-twist or bending-twist coupling. This force differential can be used to passively change the blade twist distribution. This concept has been extensively investigated by several researchers during the past two decades and several designs incorporating passive twist control have been proposed. The detailed survey of the literature provided in the following chapters suggests that there is still room for improvement in passive blade twist control applications for tiltrotor aircraft. 


\section{CHAPTER 2}

\section{A Survey of Passive Blade Twist Control Applications}

Investigations of the passive blade twist concept started in early 1980's. Bauchau and Bryan. [7] applied the concept to XV15 tiltrotor aircraft with an extension-twist coupled rotor blade. Two designs were considered. The first design involved constraints on all the stiffness and mass properties, which were matched to the baseline design. This approach resulted in $5 \mathrm{deg}$ twist change with an assumed rotor speed difference of $15 \%$ between hover and cruise modes. In the second design the constraints on the stiffness were relaxed, but the mass and c.g. location distributions were kept the same as the baseline. This approach resulted in 2 deg twist change over the same $15 \%$ speed difference.

Reference [44] addressed the issue of whether the twist deformations required for the design of full-scale extension-twist-coupled tilt-rotor blades could be achieved within material design limit loads. Extension-twist-coupled tilt-rotor blade designs were developed based on theoretically optimum aerodynamic twist distributions. The designs indicated a twist rate requirement of $0.333 \mathrm{deg} /$ in over $100 \% \mathrm{rpm}$ rotor speed change for an XV15 baseline design. Extension-twist coupled circular tubes representative of the rotor spar were manufactured and tested. The specimens were 1.64 in in diameter and 10 in in length with several hygrothermally stable layups. The results of the investigation showed that a $\left[20^{\circ} /-70_{2}^{\circ}\right]_{s}$ layup is sufficient to satisfy the design requirements with 0.384 to $0.487 \mathrm{deg} /$ in twist rates. 
The feasibility of the passive blade twist control concept has been investigated in Ref. [47]. First optimum twist distributions for each flight mode were determined. A linear blade twist assumption led to about $6.5 \%$ improvement in power requirement with respect to a compromise design of -37 deg linear twist, if optimum twist distributions of -20 deg in hover and -42 deg in cruise could be achieved. It was also shown that the performance improvement might translate to a $600 \mathrm{lb}$ increase in the payload capability. Three different rotor blade designs were used in the analysis to improve the performance associated with conventional XV15 blade twist. The first design incorporated $15 \mathrm{lb}$ tip weight, which is stated to be typical of conventional rotor blades. For the second design the tip weight was increased to $60 \mathrm{lb}$, while in the third design no limit was imposed on the tip weight. Each blade was modeled as a NACA0012 airfoil with a D-spar and 20 in constant chord. Apart from the tip weights additional running weights along the span of the blade were incorporated as needed. An optimization technique was utilized based on maximizing the twist deformation at each section along the blade subject to material strength limitations. To meet material strength limitations the maximum flapwise and inplane bending moments together with maximum centrifugal load were assumed to act simultaneously on the structure. The maximum values for the loads were obtained based on earlier XV15 test data. The design parameters included geometric characteristics for the cross-section together with the laminate ply angles, the tip weight and the running weight. All three optimized designs achieved $6.5 \%$ cruise performance improvement, as the initial twist distributions were adjusted to match the optimum 42 deg linear twist in cruise mode. The optimization resulted in hover performance improvements of 2.6, 4.8 and 5.8\% for the first, second and the third 
design, respectively demonstrating the feasibility of the passive blade twist control concept for tiltrotor applications.

The work of Ref. [47] was further expanded in [40, 39] which included an experimental study leading to a "proof-of-concept" for extension-twist coupled rotor blades. A set of composite rotor blades with NACA0012 airfoil design were manufactured using $\left[0^{\circ} / 90^{\circ}\right]$ cloth pre-preg, rotated 20 deg and tested in the helicopter hover facility at NASA Langley. The designs had an initial twist of -8.25 deg over 55 in span and a solidity of 0.0982. Two configurations were considered, one referred to as ballasted configuration with a total weight of $2.6 \mathrm{lb}$, where additional tungsten weights were added along the blade span, and unballasted configuration with a total weight of $1.44 \mathrm{lb}$, where no additional masses were added to the system. Details of the rotor blade cross-section are shown in Figure 2-1.

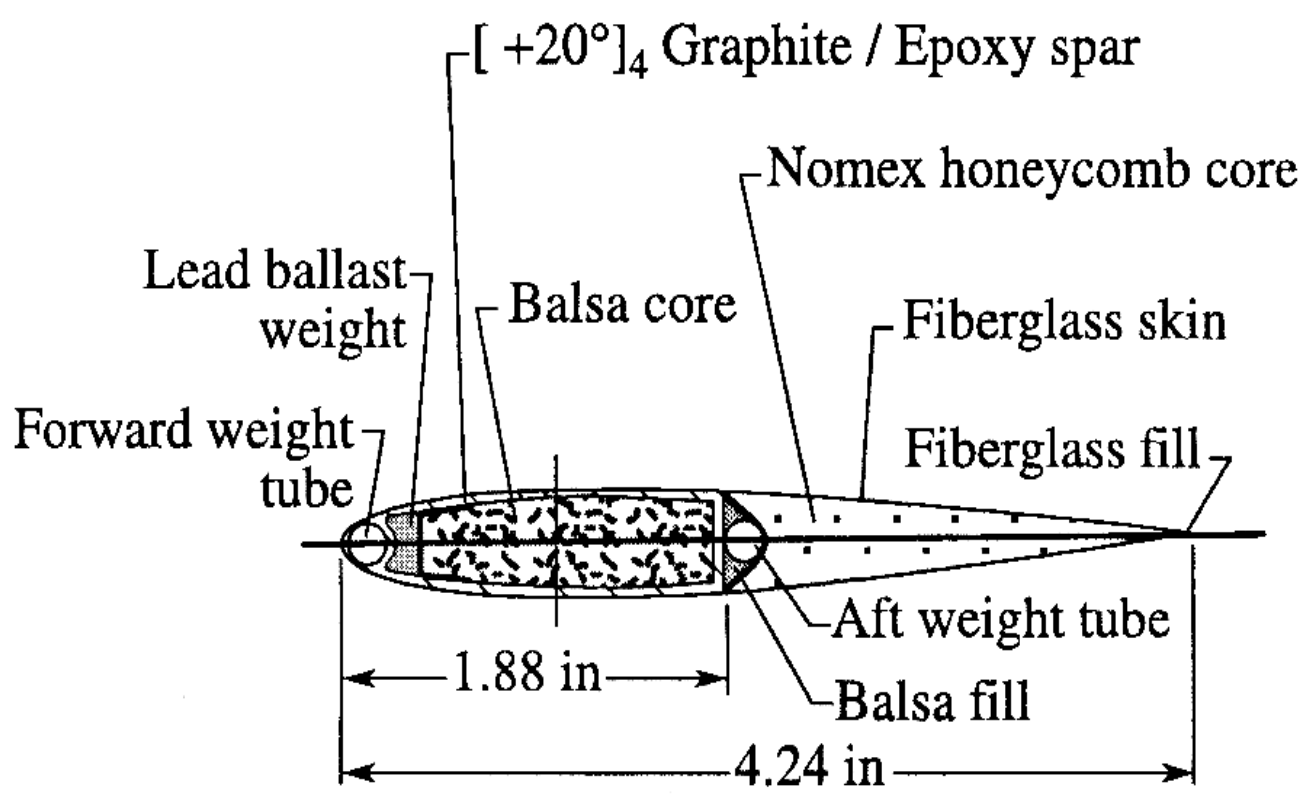

Figure 2-1. Rotor Blade Cross-Section [39] 
A geometrically nonlinear finite element analysis based on MSC/NASTRAN was also conducted to validate the tests. The results of the hover tests and finite element analysis point to a twist change of $2.54 \mathrm{deg}$ and $5.24 \mathrm{deg}$ over $100 \%$ rotor speed change for unballasted and ballasted configurations, respectively. Although the study demonstrates the feasibility of the passive blade control, a typical tilt-rotor blade would require much higher twist rate changes. The authors suggested the addition of tip masses to reach high twist rates for practical applications.

Nixon [45] focused on improving the available analysis tools in order to better model the aeroelastic response and stability of tiltrotor aircraft with elastically coupled composite rotor blades. A new finite element based tiltrotor aeroelastic analysis methodology was derived based on Hamilton's principle. The developed methodology was an improved version of the one used for UMARC (University of Maryland Advanced Rotor Code) and included additional degrees of freedom associate with the hub model, new terms from the anisotropic beam modeling, a new formulation for the precone effects and a new tiltrotor configuration model. The analysis tools were evaluated by investigating elastically coupled rotor blade configurations and making comparison with the baseline design of the XV15. In one of the cases a passive twist control concept with extension-twist coupled composite rotor blades was considered. The analysis showed that the optimum twist distribution in hover is $25 \mathrm{deg} / R$ and results in $11 \%$ reduction in power requirement compared to the baseline system, where $R$ represents the nondimensional blade span. On the other hand in the airplane mode the optimum twist distribution is the baseline twist distribution up to $0.4 R$ and $42 \mathrm{deg} / R$ from $0.4 R$ to $1.0 R$. This represents $1 \%$ improvement in the power requirement. 
This is expected since the baseline system was already optimized for the airplane mode as the vehicle operates mostly in this mode.

The extension-twist coupled composite blade, shown in Figure 2-2, was obtained by forming a NACA0012 airfoil entirely of $\left[0^{\circ} / 90^{\circ}\right]$ graphite/epoxy woven cloth. The off-axis ply angle was chosen as $20 \mathrm{deg}$, which was claimed by the author to give high extensiontwist coupling as well as allow for the system torsional and flapwise bending stiffnesses to be maximized simultaneously within the baseline limits. The elastic tailoring was applied up to $0.8 R$ of the blade from its tip. The remaining $20 \%$ span from the root was kept untailored to account for the high root forces. To meet the desired amount of twist distribution changes, additional tip masses were included. The mass values were chosen to be realistic and were within the load carrying capabilities of the blade. Figure 2-3 depicts the required initial builtin twist distributions for different tip-masses.

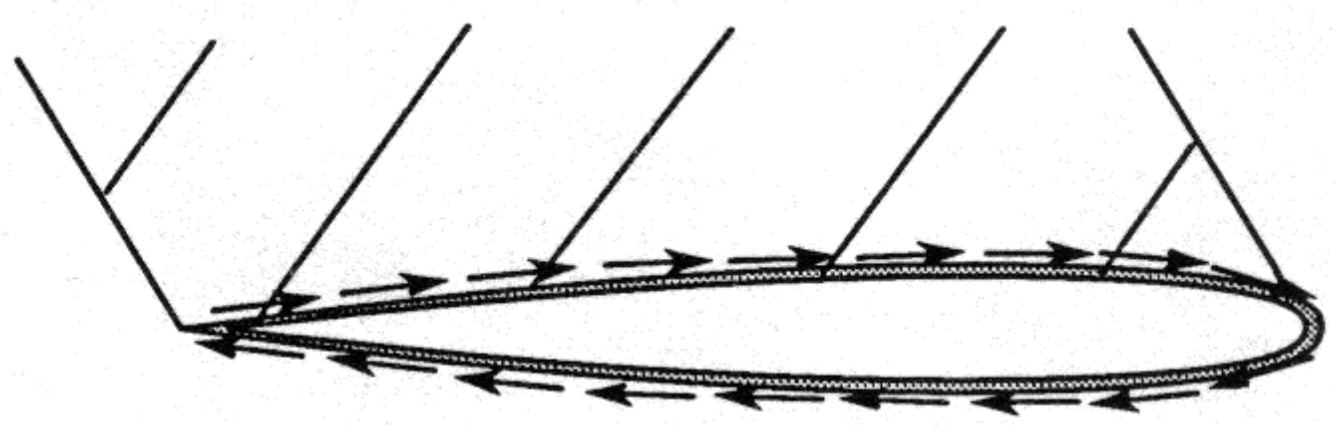

Figure 2-2. Composite Blade Cross-Section [45] 


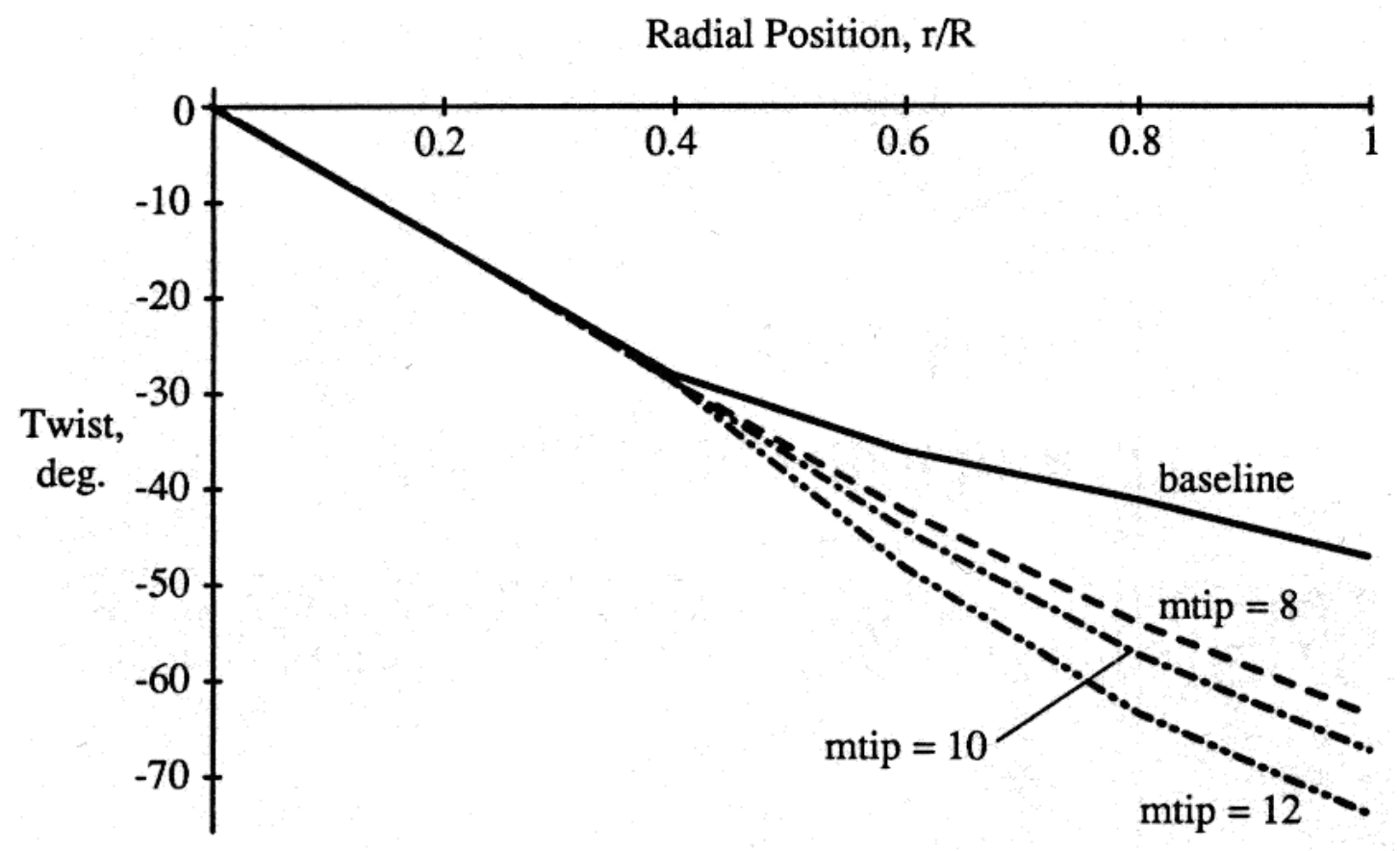

Figure 2-3. Extension-Twist Coupled Blade Twist Distributions with Varying Tip Masses, (Non-rotating) [45]

The proposed section resulted in 6 to $7.5 \%$ increase in performance in hover and $1 \%$ increase in forward flight with respect to the baseline design with the addition of tip masses to achieve the desired level of centrifugal forces required to passively change the twist distribution. The section ended up giving 8 to $10 \mathrm{deg}$ tip rotation change between the two modes. The major drawback of the configuration is the considerable change in the stability characteristics of the vehicle. The addition of the tip masses was found to be highly destabilizing and resulted in a 40 to $75 \%$ reduction in the flutter speed of the aircraft. A $20 \%$ 
increase in wing torsional stiffness and a reduced precone to near zero were suggested as a solution to the flutter problem. Possibility of utilizing bending-twist coupled rotor blades for performance improvement of the XV15 aircraft has been also investigated by Nixon [45]. It was shown that the bending-twist coupled system had about the same performance figures as the baseline design but provides a favorable influence on the stability of the aircraft. A summary of the various approaches and other possible means of improving performance in tilt-rotor applications are given in [46].

An optimization technique was applied to improve the performance of tiltrotor aircraft with elastically tailored composite rotor blades by Hodges and Soykasap [59, 61]. The research included the development of the structural and aerodynamics models, aeroelastic analysis and the optimization. The Variational Asymptotic Beam cross-Section code (VABS) $[15,66,67]$ was used to calculate the cross-sectional stiffness properties and the nonlinear beam theory of Hodges [29] was chosen to model the rotor blade. The aerodynamics model included a lift model, which accounts for a thin airfoil with both circulatory and noncirculatory aerodynamic forces and moments, and an inflow model, which incorporates the steady state dynamic inflow theory by Peters and He [49].

Two available codes were used for the aeroelastic analysis. The steady state response was obtained by AEROSCOR (Aeroelastic Stability of Composite Rotors) [56]. The rotor system was assumed to be a hingeless gimbaled rotor system and each blade was assumed to be under cantilevered boundary conditions for the steady state response. The aeroelastic stability of the system was investigated by calculating the eigenvalues of the resulting linear ordinary differential equations. The whirl stability was checked outside the optimization procedure by using the computer code RAPID (Rotorcraft Analysis for Preliminary Design) 
[2], which assumes rigid blades. The optimization was performed based on maximizing the figure of merit in hover and axial efficiency in forward flight. The section considered in the optimization is shown in Figure 2-4. It consists of a rectangular composite torque box. The composite box is assumed to be extension-twist coupled. The possibility of performance improvement with rotor blades utilizing other type of elastic couplings such as bending-twist coupling is also investigated. The sectional mass and the leading edge balancing mass were modeled as lumped masses. The airfoil skin itself had no structural contribution to the model.

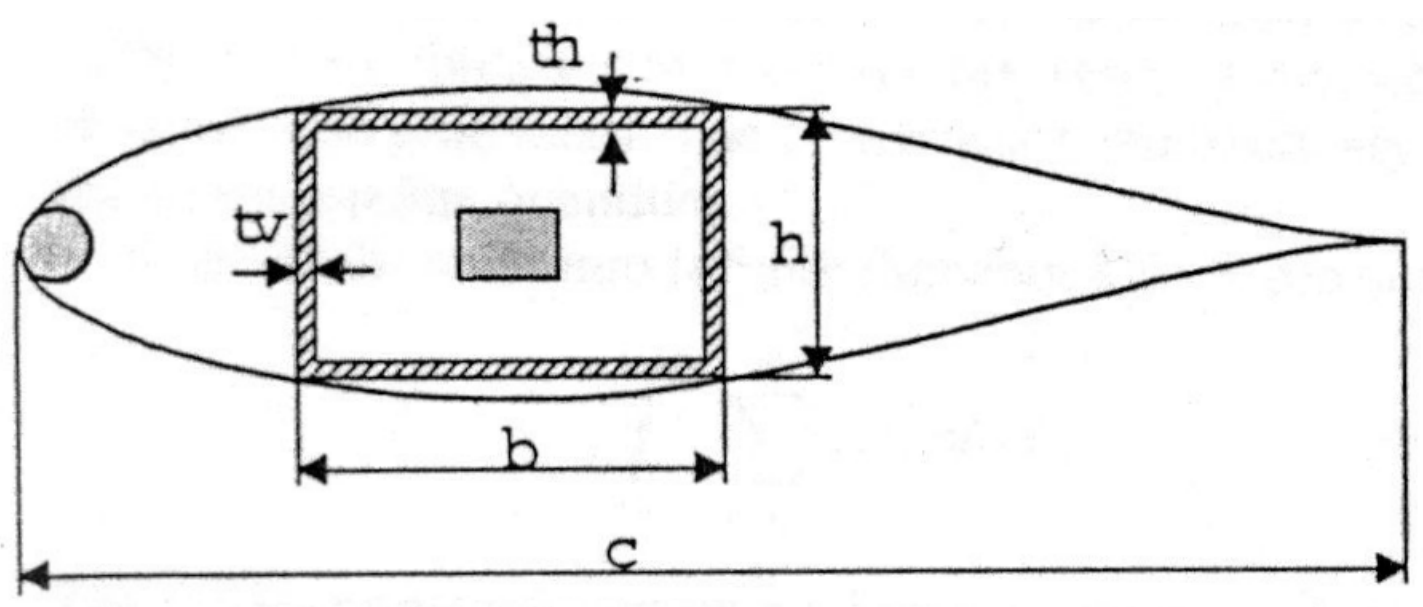

Figure 2-4. Cross-Sectional Model [59]

The design variables considered for the optimization were ply angle, torque box dimensions, twist distributions, rotor speeds and mass distributions. To assure a realistic design, constraints were applied on blade weight (kept constant), auto-rotational inertia, 
strength (Tsai-Wu failure criteria was used), and aeroelastic stability. The box-beam geometry was limited to a thin-walled section by maintaining thickness to box width ratio less than $10 \%$. A design sensitivity analysis was performed first to define the most important design variables so that an efficient optimization procedure could be established. It was shown that the performance in both flight modes is very sensitive to ply orientation angle and the twist distribution.

The optimization was first performed on the ply angle orientation and angles of attack in hover and forward flight as constraints. Next the rotor speed was taken as a design variable. It was shown that only 0.2 to $0.8 \%$ performance increase was available with changes in the rotor speed, hence the baseline rotor speed was kept constant. In the next step the built in twist distributions were taken as design variables. It resulted in optimum distributions with about $4 \%$ increase in performance in both flight regimes. Finally the box beam design variables, nonstructural masses, the ply angle and the twist distributions were taken as design variables, adding up to 73 variables. The starting point for the analysis was chosen as the optimized section that resulted from the built-in twist distribution analysis. The resulting optimized section was a conical type rectangular box with 22 deg ply orientation and $33.5 \mathrm{deg}$ initial twist. The final section represented a $4.34 \%$ improvement in the objective function with respect to the XV15 baseline design utilizing extension-twist coupled rotor blades. The optimized system was shown to be flutter free and aerodynamically stable. The conical form of the blade load carrying member is an expected result as the need for high torsional stiffness reduces towards the rotor blade tip and probably helped achieving an aerodynamically stable system that can utilize passive twist control without any weight penalty. 
The performance improvement in hover associated with a system utilizing bendingtwist coupled rotor blades were reported to be $1.42 \%$ to $0.88 \%$ in hover for flap-torsion coupled and lag-torsion coupled rotor systems, respectively. Slightly better improvements were observed in forward flight. However, the overall performance improvement compared to the extension-twist coupled case was about $40 \%$ lower.

The major drawback of the analysis reported in [59] is the simplified cross-sectional model. Also a more complete aeroelastic analysis involving wing/pylon/rotor system could be employed to validate the results.

This work was revisited in [60] and an optimization technique referred as the "Inverse Method" was utilized for the objective function. In this method the rotor blade stiffness distributions, which were assumed to be constant over the blade span, were taken as design variables. Subsequently geometric properties of the cross-section, which can produce the desired stiffness distribution, were determined. Constraints were imposed on positive definiteness of the stiffness matrix, non-rotating natural frequencies of the beam, angle of attack and material failure. Based on the optimized stiffnesses, different composite materials for the rotor were studied to obtain a maximum performance in both flight regimes. The objective function was based on maximizing axial efficiency in forward flight and figure of merit in hover, and they were reflected in the objective function with equal weighting ratios. This reportedly emphasizes an equal importance in forward flight and hover performance. Results showed that improvements in the tilt-rotor performance as high as $3.6 \%$ are possible using optimally stiffened beam. 


\section{CHAPTER 3}

\section{Present Research}

\subsection{Scope of the Present Research}

The survey of the prior work on the passive blade twist control concept reveals that the concept is most effective with extension-twist coupled rotor blades. Moreover a need is observed for a cross-section with high levels of extension-twist coupling and adequate levels of torsional stiffness to achieve required blade twist changes within $20 \%$ of rotor speed range without negatively altering the stability characteristics of the system. In the present research, availability of such a cross-section is discussed first. In particular the feasibility of "star cross-sections" is addressed. A solution to the problem is presented based on the results of the work on star cross-sections and the previous work done on the concept by other researchers. A multilevel optimization procedure, which involves a cross-sectional analysis and multibody dynamics approach, is applied to achieve optimum model system parameters for performance improvement of a tiltrotor based on the XV15 baseline design.

In the following chapters details of the proposed model and the optimization approach are presented.

\subsection{Star Cross-Sections}

The technical barrier in passive blade twist control is the large extension-twist coupling requirement together with adequate levels of torsional stiffness of the rotor blade. 
The development of an efficient elastically tailored family of extension-twist coupled composite beam provides a basis to overcome this technical barrier. The "star cross-sections" [21] were initially proposed as a solution for preserving the high level of extension-twist coupling of flat strips while increasing the bending and torsional stiffnesses. The variation of extension-twist coupling with stiffness for various tailored configurations illustrates the underlying concept behind the development of star cross-sections, Figure 3-1. On one end flat strips are characterized by large extension-twist coupling and low torsional stiffness whereas on the other end closed cells have low extension-twist coupling but high torsional stiffness. The star cross-sections with closed circular core were initially proposed to combine open and closed cell properties providing a compromise between an increase in torsional stiffness and decrease in coupling. The motivation behind the research is the verification of the schematic illustrated in Figure 3-1 and the accurate determination of the magnitude of coupling and torsional stiffness associated with a given star cross-section configuration.

The feasibility of such cross sections for passive blade control applications has been extensively investigated. The main mechanism producing extension-twist coupling in composite laminated strips is the extension-shear coupling of the off-axis plies and it can be maximized using anti-symmetric stacking sequences. A nonlinear finite displacement analysis for extension-twist coupled laminates was presented in [1]. The torsional stiffness of such laminates was determined in [41] based on geometrically nonlinear analysis. A number of test methods and apparatuses was developed in order to measure the twist angle under axial loading $[27,32,33]$. The first star cross-sections, obtained by joining several extensiontwist coupled laminated strips [21], as depicted in Figure 3-2, were manufactured to maintain the large extension-twist coupling of the individual flat strips while increasing the relative 
bending and torsional stiffness. However, the increase in torsional stiffnesses were found to be insufficient to match that of closed cells.

The star configurations combining a circular core with varying number of flat strips referred to as fins, shown in Figure 3-3, were developed in order to increase torsional stiffness. Configurations with variable diameter, wall thickness, length and number of fins leading to tailorable torsional stiffness and extension-twist coupling with constant axial stiffness, were manufactured and tested as reported in [37].

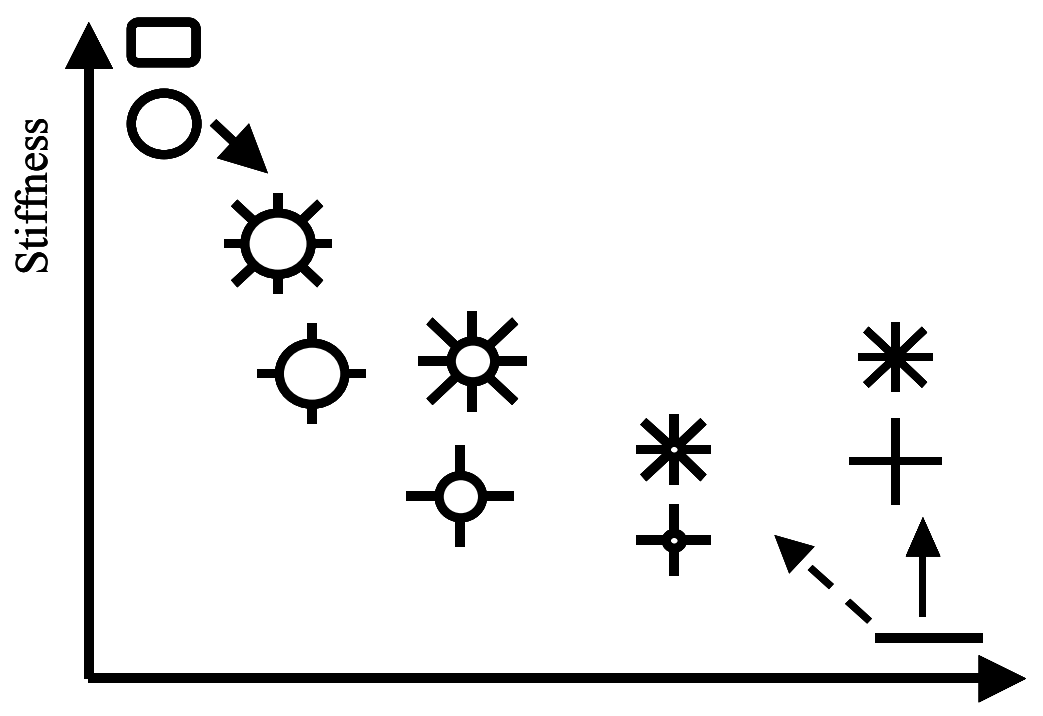

Coupling

Figure 3-1. Extension-Twist Coupled Cross-Sections with Tailored Stiffness 


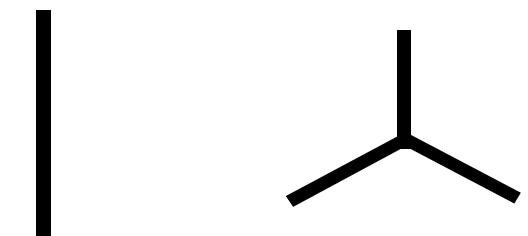

$n=2$

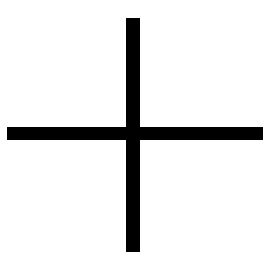

$n=4$

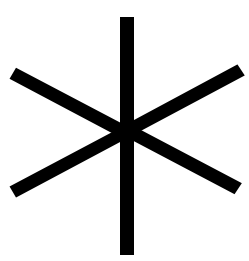

$n=6$

Figure 3-2. Star-Beam Cross-Sectional Configurations

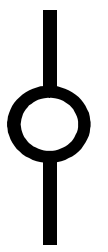

$n=2$

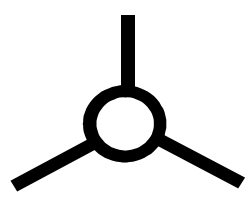

$n=3$

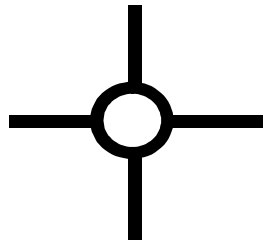

$n=4$

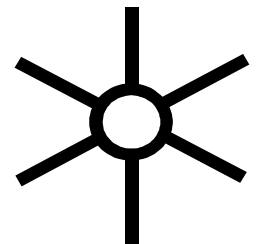

$n=6$

Figure 3-3. Modified Star Configurations with Cylindrical Central Core

A typical test result is shown in Figure 3-4. The set of data with high twist rate (solid symbols) were obtained using a star configuration without a circular core. The effect of the circular core on the structure for the same axial stress is depicted by the second set of data (open symbols) with lower twist rate. At $115 \mathrm{MPa}$ the twist rate drops by $60 \%$. 


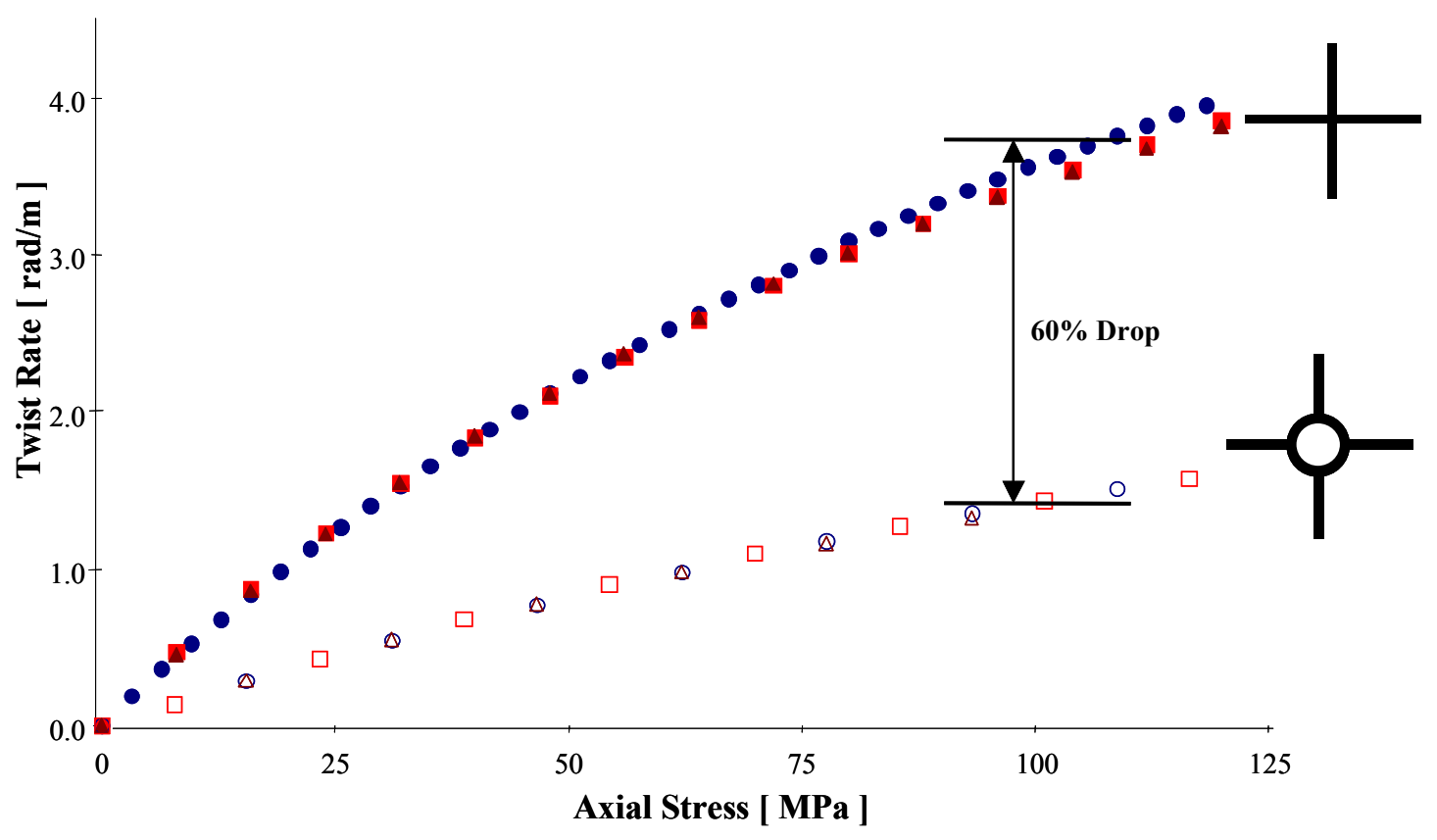

Figure 3-4. Comparison of Extension-Twist Coupling from Star and Modified Star Configuration with Antisymmetric Layup

A parametric study on the family of star cross-sections was performed using SVBT, a linear finite element cross-sectional beam analysis code based on the work of Borri and Giavotto [26]. A mesh simulation for an eight-finned cross section is depicted in Figure 3-5. The simulation included 832 solid 9-node elements with a total of 11,136 degrees of freedom. A hygrothermally stable $\left[20^{\circ} /-70_{2}^{\circ} / 20^{\circ} /-20^{\circ} / 70_{2}^{\circ} /-20^{\circ}\right]$ stacking sequence [64] is used for the fins and half of the stacking sequence, $\left[20^{\circ} /-70_{2}^{\circ} / 20^{\circ}\right]$, is used for the circular core. 
The structural parameters used for the study include the number of fins, the width of the fins, the thickness and the radius of the circular core. The amount of material used is kept constant in order to provide a consistent basis for comparison of cross sections. Since the layup is unchanged, this is equivalent to keeping a constant axial stiffness. Starting with a circular section, the number of fins is allowed to grow, causing the radius of the core to decrease.

The results of the parametric study are shown in Figure 3-6. It is observed that the number of fins has a negligible effect on the extension-twist coupling behavior and torsional stiffness. This is because the enclosed area of the core is the predominant parameter that controls the behavior. While a closed cross section is effective in increasing torsional stiffness, it constrains the large extension-twist coupling created by the fins.

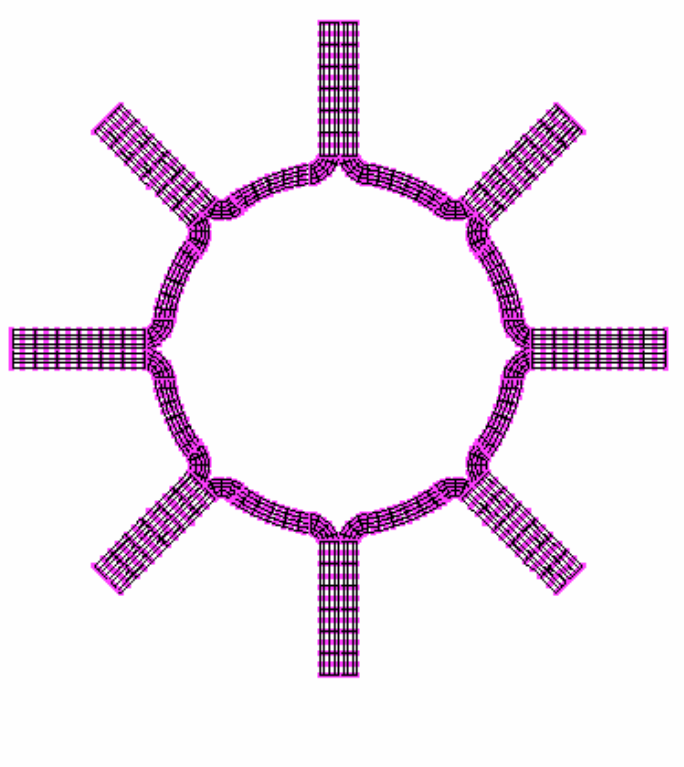

Figure 3-5. FE Model of an Eight-Finned Star Section with a Circular Core 


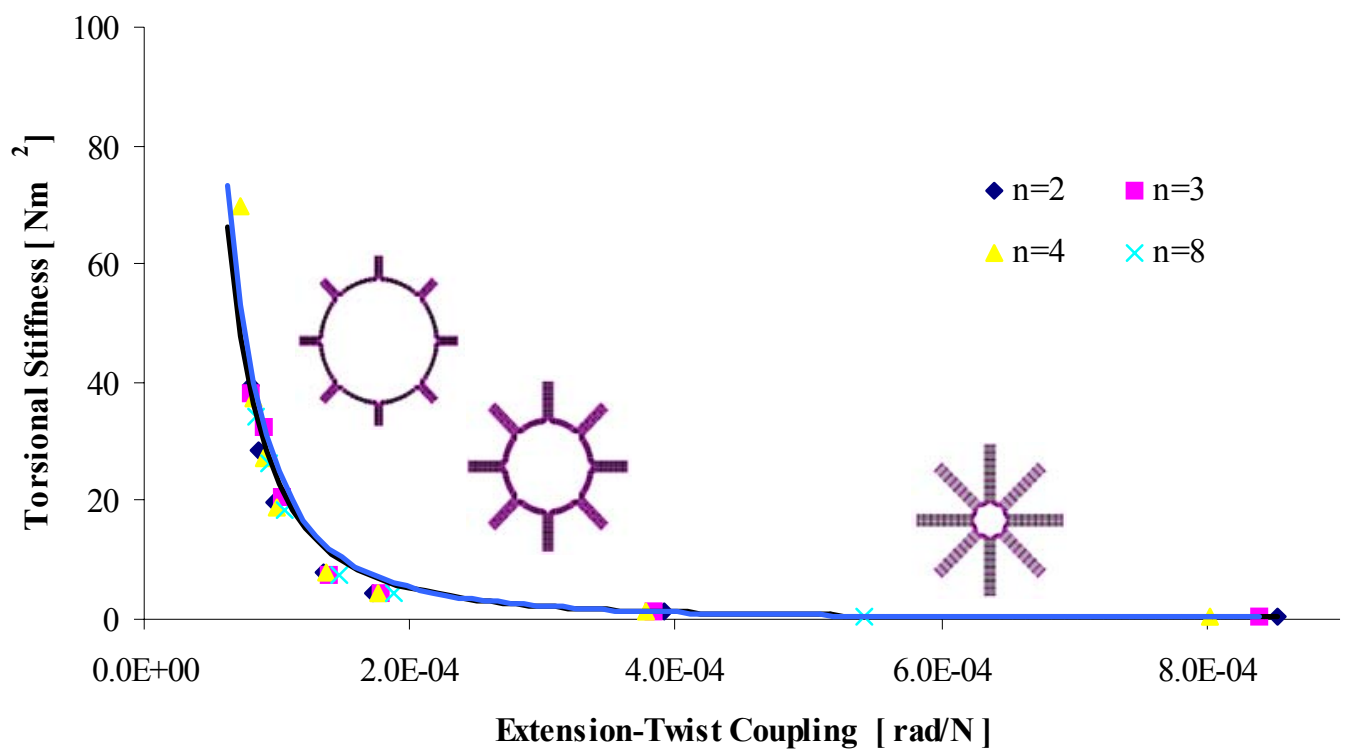

Figure 3-6. Torsional Stiffness vs. Extension-Twist Coupling of Star Configurations with Varying Number of Fins (n) and Core Radius

The relative magnitude of the negative trend between the increase in torsional stiffness and decrease in extension-twist coupling is depicted in Figure 3-7. Normalized torsional stiffness, $G J$ and extension-twist coupling, $E T$, with respect to a star configuration with four fins, $(n=4)$, is plotted against the core radius on a semi-log scale. The vertical dashed line in Figure 3-7 shows that a 13 times increase in torsional stiffness is associated with an $80 \%$ decrease in extension-twist coupling. It should be noted that the data points at each core radius for different number of finned sections are in close proximity to each other. This shows that the circular core area mainly controls the section's behavior, and the fins are not as effective in increasing extension-twist coupling as intended. 


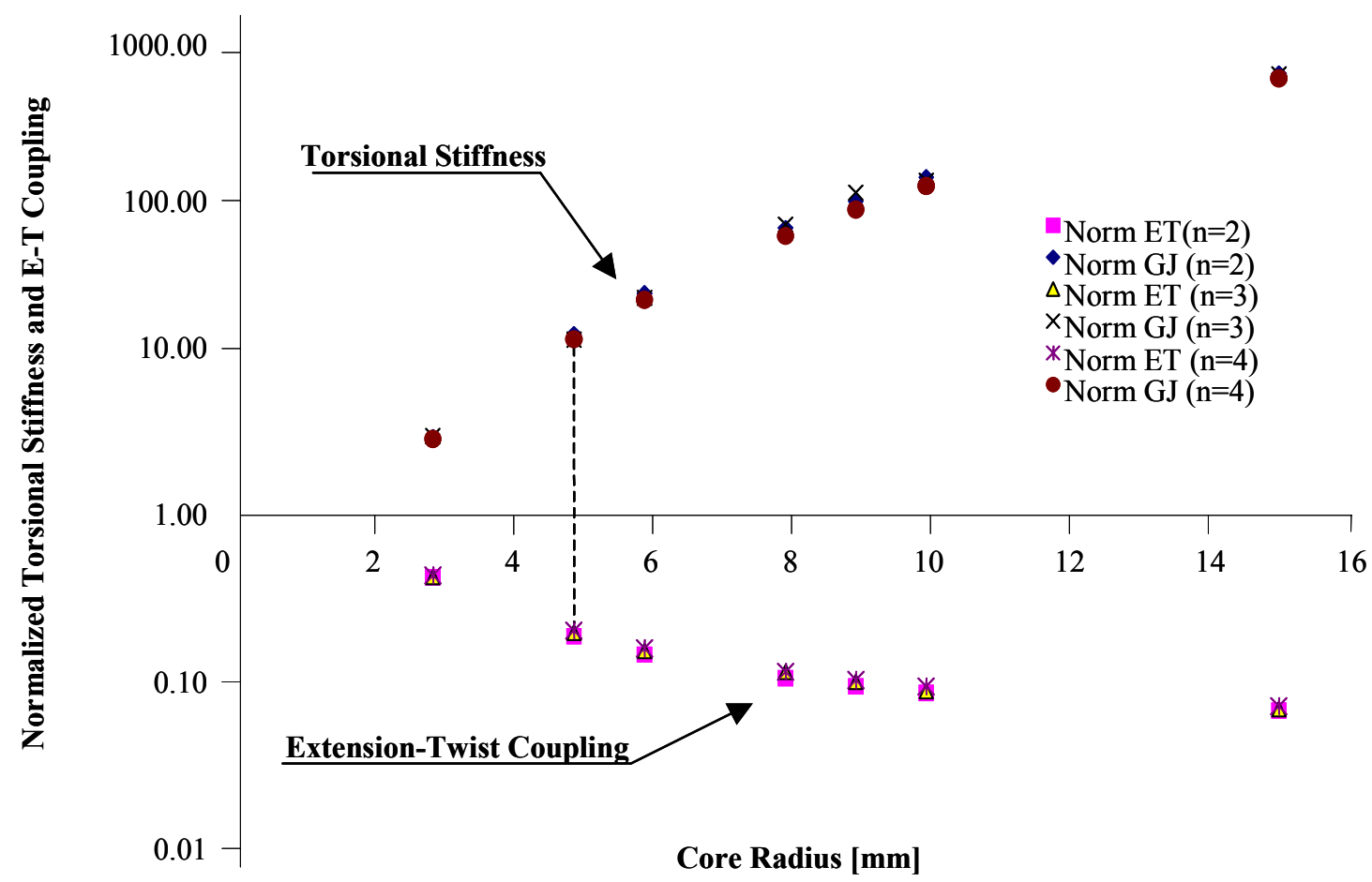

Figure 3-7. Comparison of Normalized Torsional Stiffness and Extension-Twist Coupling with respect to 4-Finned [ $n=4$ ] Case

The effect of the circular core on the behavior of the structure can be further evaluated using the closed form solution of Berdichevsky, Armanios and Badir. [10] for thinwalled closed sectioned beams. In this formulation the torsional stiffness and extension-twist coupling for a circumferentially uniform cross-section are given as in Eqn 1, where $A, B, C$ are axial, coupling and shear stiffnesses, respectively and are constant for a circumferentially uniform cross-section. $A_{e}$ and $L$ represent the enclosed area and circumference of the cross- 
section, respectively. Hence for a closed section $\left(E T A_{e}\right)$ and $\left(G J \frac{L}{A_{e}^{2}}\right)$ should be constant for a given material and layup regardless of the shape of the cross-section.

$$
\begin{aligned}
& E T=\frac{B}{B^{2}-A C} \frac{1}{A_{e}} \\
& G J=C \frac{A_{e}^{2}}{L}
\end{aligned}
$$

The variation of these stiffness constants with varying core radius for a 4-finned star cross-section is shown in Figure 3-8. It can be seen that the torsional stiffness parameter stays constant with variation in core radius. This shows that the change in the torsional stiffness of the cross-section is predominantly determined by the enclosed area. The extension twist coupling parameter in Figure 3-8 is not as uniform as the torsional stiffness but remains within $30 \%$. This points to the fact that although the coupling of the section is mainly controlled by the enclosed area there is still a contribution of the fins in the extension-twist coupling of the cross-section. 


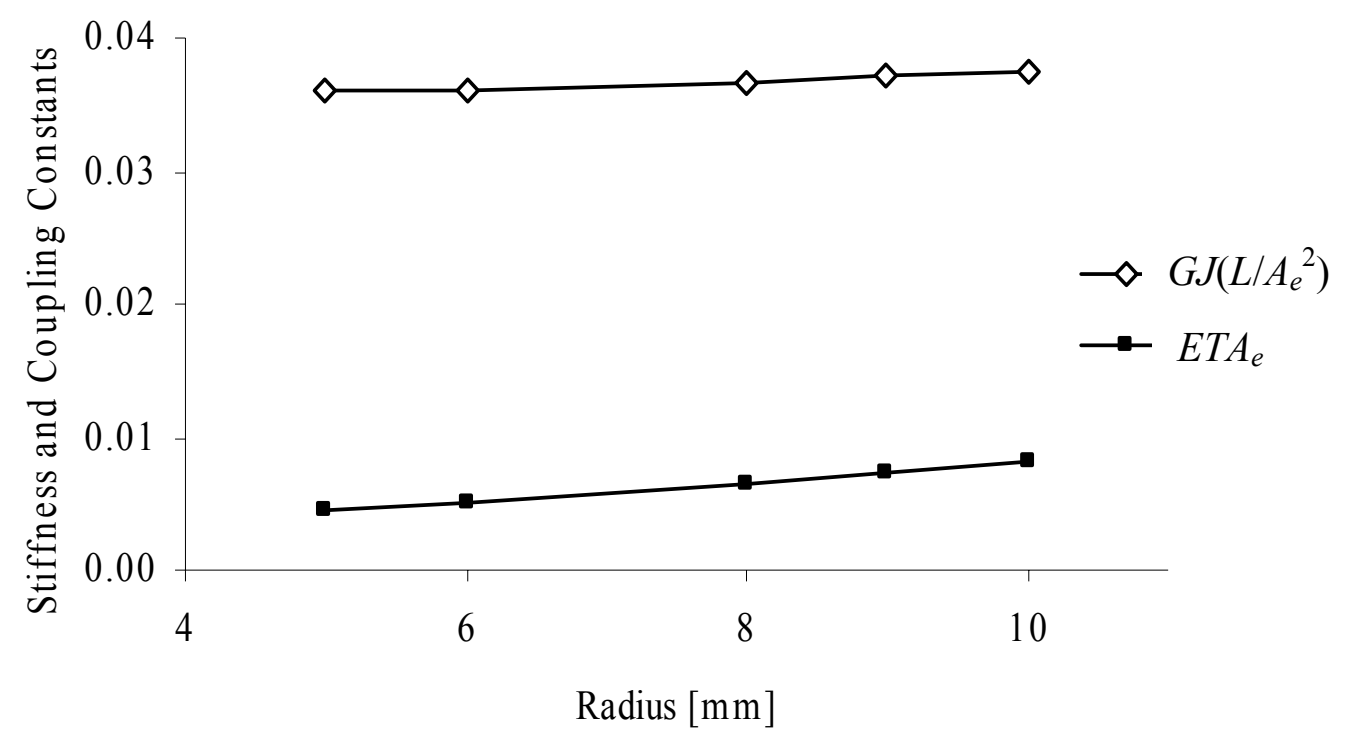

Figure 3-8. Torsional Stiffness and Extension-Twist Coupling Constants

In conclusion the star cross-sections are not as effective in achieving the passive twist control level required for rotor blade stability due to the following reasons.

- There is a negative trend between increase in torsional stiffness and decrease in extension-twist coupling

- The sectional behavior is mainly controlled by the circular core and the fins do not serve the intended purpose of increasing extension-twist coupling

- Furthermore star cross-sections have to be combined with a suitable skin-type structure, to provide an airfoil shape for the blade without interfering with the stiffness and coupling of the base structure. This represents an additional challenge to the implementation of star cross-sections in rotorcraft applications. 


\subsection{Proposed Model}

The research on passive blade twist control concept for tiltrotor applications has been discussed in previous sections. The following remarks should be restated in order to put the proposed model for this work into context.

- A realistic rotor blade requires some means for amplifying the extension-twist behavior, such as additional mass. This provides higher levels of centrifugal loads on the structure; however the additional mass causes aeroelastic instabilities, which may require further alterations to the rotorcraft system as a whole.

- It has been shown that it is theoretically possible to enhance the performance of a tiltrotor aircraft using a generic simple box beam type model. The optimum box beam is conical reflecting that the torsional stiffness requirement is not the same along the blade span, being higher towards the root of the system.

- Feasibility studies on the star cross-sections showed that they did not meet practical requirements for twist control.

In the present research an answer is sought for the question "Is it possible to achieve performance improvement using a practical and realistic rotor blade cross-section without additional weight penalty?"

The first step in answering this question is the investigation of the availability of any means that can be used to amplify the extension-twist behavior of a conventional rotor blade. A typical rotor blade carries some non-structural masses, such as the leading edge balancing mass. If this mass could be shifted to the blade tip and allowed to slide anywhere else with respect to the blade frame, then this would lead to an efficient and effective way to transfer 
the axial load due to centrifugal forces to the tip of the blade without any weight penalty. One way of achieving this concept is to design the balancing mass as a distribution of stacked disks or particulates, within a friction-free housing with a closed end stopper at the blade tip. An illustration of the concept is given in Figure 3-9 and is referred to as "sliding mass concept" throughout the text.

The effect of the sliding mass concept on the blade elastic twist distribution is depicted in Figure 3-10. The rate of change of elastic twist distribution zeros out towards the blade tip in an extension-twist coupled composite rotor blade with a conventional mass distribution. This is due to the vanishing of the centrifugal forces towards the blade tip. The sliding mass concept proves to be beneficial at this point by providing a positive change of elastic twist especially at the blade tip. It effectively shifts the centrifugal force to the blade tip where elastic tailoring can be more effective due to the airload distribution over the rotor blade and increases the possibility of meeting the desired twist rate without any weight penalty. 

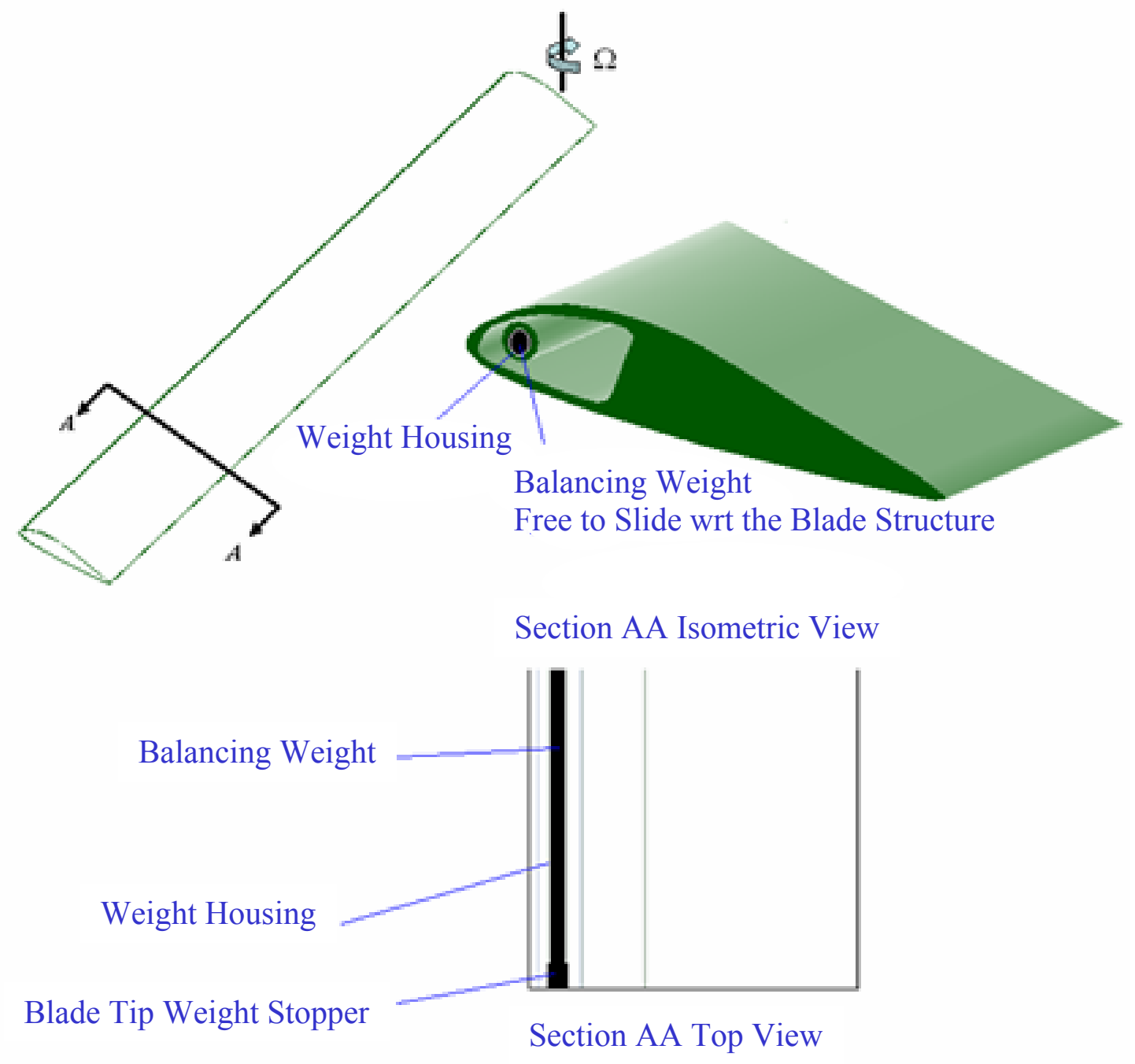

Figure 3-9. Sliding Mass Concept 


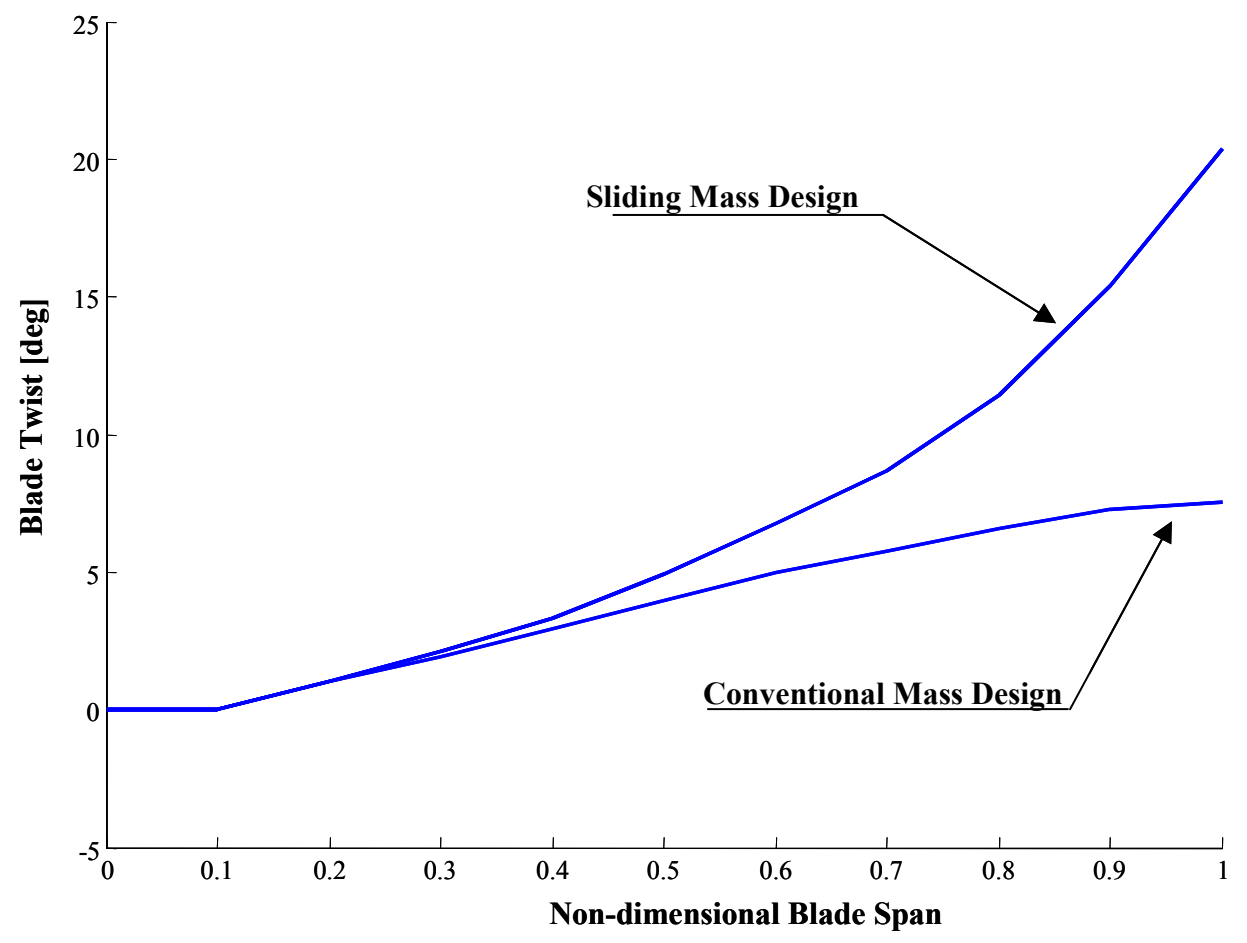

Figure 3-10. Blade Elastic Twist Comparisons

It should also be noted that the rotor blade of an XV15 has a conical shape as illustrated by the normalized blade thickness plot in Figure 3-11. This is consistent with the conical shaped optimum box beam model reported in [59]. Hence the sliding mass concept together with the conical blade profile may provide a solution to the performance enhancement in a tiltrotor aircraft via passive blade control. 


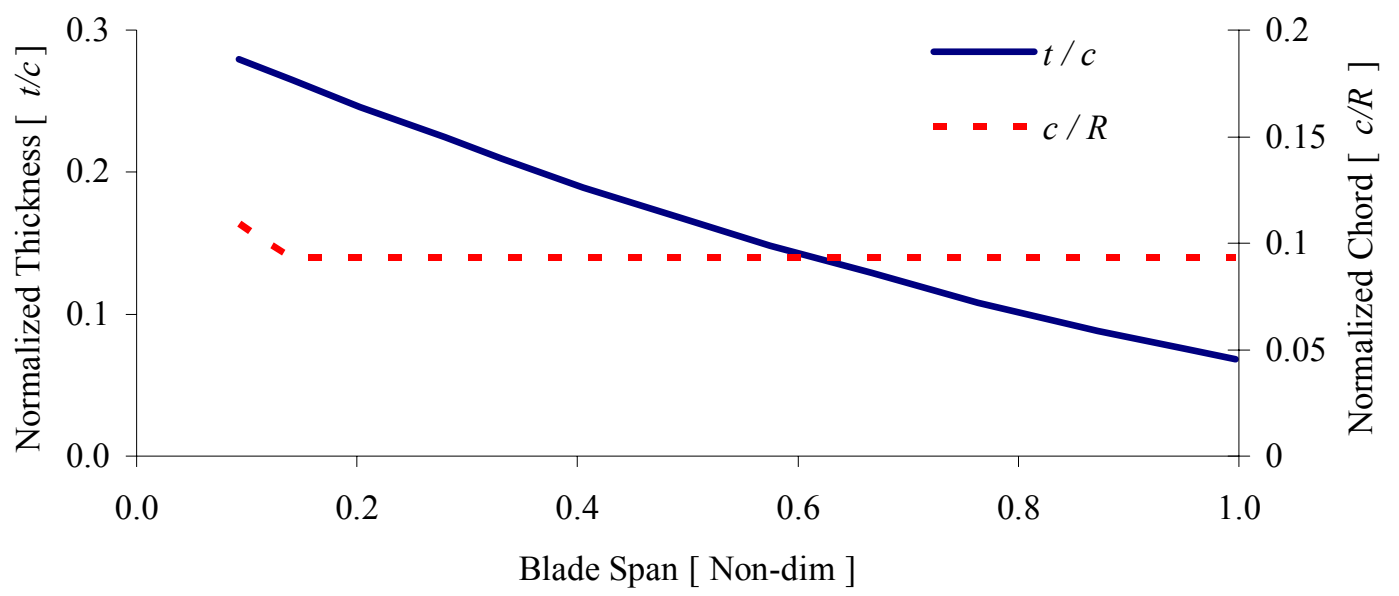

Figure 3-11. XV15 Thickness to Chord Ratio [59]

The aim of this work is to improve the performance of a tiltrotor aircraft with practical extension-twist coupled rotor blades using the sliding mass concept. The finite element based multibody dynamics code DYMORE [8] is used to model the system. The cross-sectional modeling is performed using VABS [15, 66, 67]. Finally an optimization technique is performed on the rotor blade in order to improve the performance of the aircraft. 


\subsubsection{Rotor Blade Cross-Sectional Analysis}

Having one dimension much larger than the other two, rotor blades are usually treated as beams. However, the complex geometries of blade cross-sections and the anisotropic nature of the composite material systems make the accurate representation of the rotor blade as a (1D) beam model a rather challenging task.

A comprehensive review of the earlier work on cross-sectional modeling of composite rotor blades has been given by Hodges [30]. One of the earliest is by Mansfield and Sobey [42] where they modeled the rotor blade as a composite hollow tube. Wörndle [65] used a finite element based approach to determine the shear center and warping functions for a composite rotor system made of transversely isotropic material. Subsequently Rehfield [54] proposed a method where the rotor blade is approximated by a closed box beam, whose torsional warping functions can be determined analytically. Bauchau [4] developed a beam theory, where the analysis was restricted to closed multi-celled thin-walled beams with a transversely isotropic material system. Later Bauchau, Coffenberry and Rehfield extended this work to include general orthotropy [6]. Giavotto et al. [26] proposed a two-dimensional finite element based procedure to determine the cross-sectional properties of a system with general anisotropy. Borri and Mantegazz [12] and Borri and Merlini [13] extended this work to include nonlinear deformation. The analysis was later successfully implemented by Bauchau in the SVBT computer code providing the ability to analyze practical, anisotropic, inhomogeneous initially untwisted rotor blade cross-sections.

Smith and Chopra [57] proposed a direct analytical beam formulation for predicting the effective stiffness and corresponding deformation behavior of tailored composite boxbeams and experimentally validated their analysis. Berdichevsky, Armanios and Badir [10] 
developed an analysis using the variational asymptotic method in order to derive the governing equations of anisotropic, thin-walled beams with closed sections. The analysis was based on an asymptotic analysis of two dimensional shell theory and resulted in closed form expressions for the beam stiffness coefficients, stress and displacement fields. Later work by Volovoi and Hodges [62] corrected this work and were able to consistently include shell bending strain measures.

Recently Cesnik, Hodges and Sutyrin [16] developed a finite-element based asymptotically correct methodology, including geometrically nonlinear 3D elasticity for the cross-sectional analysis of initially curved and twisted, non-homogenous, anisotropic beams. The approach is based on the variational asymptotical method [11] and has no restriction on the cross-sectional geometry (solid or thin walled, open or closed). The methodology has been successfully implemented in the computer code, VABS [15, 66, 67] (Variational Asymptotic Beam Sectional Analysis). Being the most general cross-sectional code, VABS is chosen as the analysis tool for the current work.

\subsubsection{Multibody Dynamics Modeling for Rotorcraft Applications}

Historically the rotorcraft industry relied on custom in-house-designed-per-aircraft analysis methods. Several computer codes, DYN4-DYN5 [23], PASTA [38] and CAMRAD $[35,36]$, were used by Popelka [51] to correlate analysis and test data for $1 / 5$ scale V22 aeroelastic model. These codes are based on simplifying assumptions to reduce analytical complexity and computational cost. Although relatively detailed structural designs can be 
represented by some codes such as CAMRAD and UMARC [31], still they are limited to rotor analysis and depend on modal reduction to reduce the model size, which cannot cover a wide spectrum of aircraft systems.

Multibody dynamics analysis, originally developed as a tool for analyzing tree-like topologies with rigid bodies, has the flexibility to handle arbitrary topologies with deformable bodies and was adapted to helicopter dynamic analysis by Bauchau et al. [8]. The formulation has been successfully implemented in the DYMORE computer code, a finite element based tool for the analysis of nonlinear flexible multibody systems. The details of the approach and applications to selected examples of rotorcraft configurations are given in [5]. The proposed approach can treat arbitrarily complex topologies and the formulations of beams and shells are geometrically exact as they account for arbitrarily large displacement and finite rotation, but are limited to small strain. Since the formulation is an extension of the finite element method to multibody systems, the algorithms readily developed for FEM analyses are directly applicable.

DYMORE uses readily developed library of elements for deformable and rigid bodies, joints, actuators and sensors to define any complex system and can perform static, dynamic, trim and stability analyses. Although the computational time is relatively higher than the traditional dedicated codes, the computational efficiency on a regular desktop PC is more than adequate.

The generality and flexibility of the code provides the opportunity to investigate fuselage-wing-drive/chain-blade interactions, which are indeed important for the stability of a tiltrotor aircraft, rather easily, accurately and efficiently. Recently the feasibility of a multibody dynamics approach has been investigated using a DYMORE model of a 1/5 scale 
wind tunnel model of the V22 tiltrotor aircraft, and the approach was found to be efficient without excessive simplifications [25]. Applications of stability analysis demonstrating the accuracy and computational efficiency of the methodology were presented in [9]. In the current research the aeroelastic and aerodynamic analysis for the proposed design will be performed using DYMORE.

\subsubsection{Tiltrotor Aircraft Optimization Studies}

A survey of applications of design optimization to rotorcraft has been recently given by Celi [14]. The interactions between engineering disciplines such as structures, aerodynamics and control, makes rotorcraft design optimization intrinsically a multidisciplinary task. An extensive survey of multidisciplinary aerospace design optimization can be found in [58].

An attempt was made to couple a free wake hover code with an optimization scheme in [53]. The approach was applied to the XV15 Advanced Technology Blade (ATB) with a square tip. About $2.6 \%$ improvement in hover performance was achieved.

McCarthy et al. [43] applied a multidisciplinary optimization procedure to improve rotor/wing performance of a high-speed tilt rotor aircraft. The objectives were chosen to be improved figure of merit (FM) in hover and axial efficiency in forward flight $(\eta)$ with minimized wing weight. The first natural frequency and autorotational inertia of the rotor blade and the wing root stresses were imposed as constraints on the system. The real part of the stability root in windmill condition was bounded to guarantee stability. The design variables included the root and wing plan form properties and wing wall thickness. The 
optimum system provided $2.3 \%$ improvement in FM in hover and $0.3 \%$ improvement in axial efficiency.

A multilevel decomposition technique was employed by Chattopadhyay et al. [17] for an efficient design optimization of helicopter rotor blades. The optimization consisted of three levels. In the first level the desired thrust coefficient $C_{T}$ was matched. In the second level the aerodynamic and aeroelastic properties were determined. Finally in the third level a cross-sectional sizing was performed to match the requirements of the second level optimization. The multilevel optimization proved to be faster compared to the "all-at-once" type of optimization techniques. Later they applied the similar multilevel optimization technique to XV15 baseline design in order to investigate the effect of blade planform properties and composite tailoring on blade aerodynamic and structural performance of a proprotor aircraft [19]. The optimization was performed in two levels. In the first level the FM in hover and axial efficiency in forward flight was improved with the design variables being the blade properties such as thickness to chord ratio, twist rate and chord. A Kreisselmeier-Steinhauser (KS) function approach was used for multiobjective optimization. In the second level the box beam cross-sectional properties were determined in order to achieve the first level optimization requirements. In this level the design variables included the ply angle orientations for the composite box-beam. The lay-up orientations are chosen from the set of $\left[0^{\circ}, 15^{\circ}, 30^{\circ}, \ldots, 90^{\circ}\right]$ to ensure manufacturability. The simulated annealing method, which is discussed in more detailed in the following chapters, was employed due to the discrete nature of the design space. The optimized system provided $7.5 \%$ improvement in FM in hover and 3.2\% improvement in axial efficiency. This work was later extended to include takeoff design requirements in the objective function [20]. 
An integrated optimization procedure (IADS), which incorporates aerodynamics, dynamics and structures into the optimization using both local and global variables, was discussed in [63]. The problem with multilevel optimization is the ability to match the local variables, e.g. composite lay-up or rotor structure wall thickness, and global variables, e.g. torsional and bending stiffnesses. The upper level optimization may require a certain combination of bending and torsinonal stiffnesses. However, it is not certain if the lower level optimization may satisfy this requirement with the given set of local variables. Nevertheless the technique proposed was demonstrated on several examples and proved to be very effective.

Ganguli and Chopra [24] used an optimization technique to tailor composite rotor blade for minimum vibratory loads and improved aeroelastic stability in forward flight. Rajadas [18] used a multidisciplinary optimization technique on a composite tiltrotor aircraft. The rotor box-beam model captured both inplane and out of plane warpings. The objective was improved figure of merit in hover and axial efficiency in forward flight. The Kreisselmeier-Steinhauser function approach was used to model the multiobjective optimization as an unconstrained optimization problem. The design variables included the blade plan form properties and the composite box lay-up. A 3.7\% improvement in FM and $5.5 \%$ improvement in $\eta$ with respect to the baseline XV15 design were reported.

Recently a composite tiltrotor optimization was performed by Soykasap and Hodges [61] to improve performance. The baseline design of XV15 blade was modeled as a composite box-beam. The blade plan form properties together with the box beam geometric dimensions and composite lay-up orientations were chosen as design variables. Later an inverse optimization method was adopted for the same system [60]. The upper level 
optimization used the blade stiffness parameters as design variables and in the lower level, box-beam system parameters providing the optimum upper-level target stiffness parameters were sought after. 


\section{CHAPTER 4}

\section{Modeling of the Proposed Design}

\subsection{Blade Cross-Sectional Model}

A generic cross-sectional model for the rotor blade has been constructed using VABS. The mesh generator and output interface SVAB written by Bauchau using the DYMORE input format has been utilized for the analysis. The interface can be easily automated for optimization purposes. A screen printout from the VABS cross-sectional model is shown in Figure 4-1. The model consists of a Graphite/Epoxy composite D-spar and skin and a balsa filling for the aft section. The corresponding material properties are tabulated in Table 4-1. NACA00XX series airfoil shapes have been used for blade modeling.

A Matlab ${ }^{\circledR}$ subroutine was written and can generate the blade cross-sectional model for a desired NACA00XX series airfoil and geometric parameters, such as spar/skin thickness and D-spar width, execute VABS and document the results. This subroutine plays a critical role in integrating VABS into an optimization process.

A typical VABS model consisted of 560 elements with over 7000 degrees of freedom. The code outputs the sectional stiffness, compliance and mass matrices, and takes into account the effect of built-in twist on the cross-section. The model stiffness calculations are performed about the quarter chord. The code also provides the stress state at points throughout the cross-section, a valuable tool for subsequent stress analysis. 


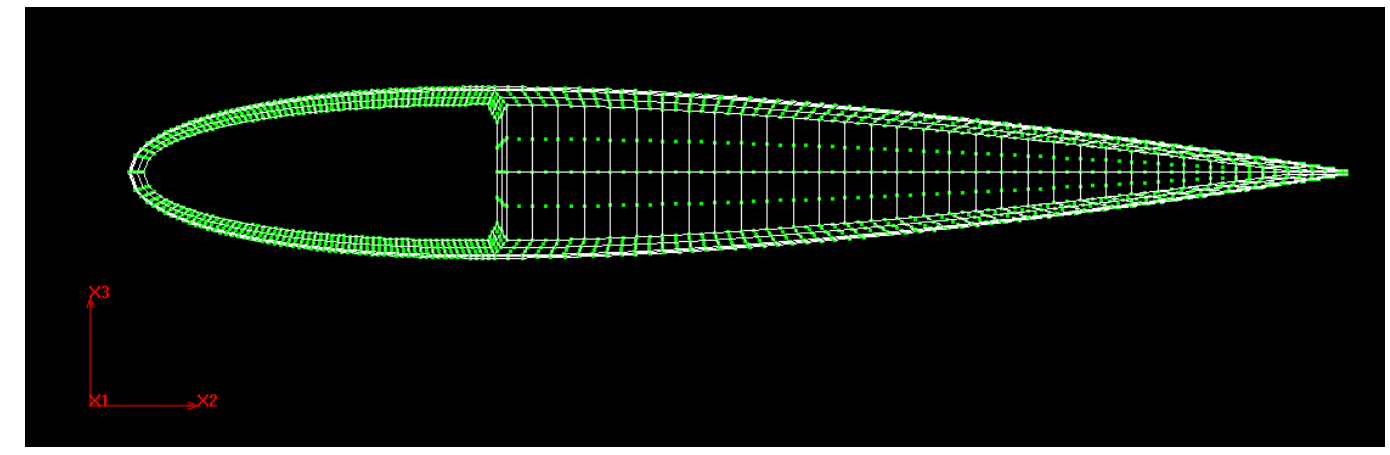

Figure 4-1. Blade VABS Cross-Sectional Model

Table 4-1 Material Properties

Graphite/Epoxy

$\begin{array}{lll}E_{11} & 138.00 & \mathrm{GPa} \\ E_{22} & 8.96 & \mathrm{GPa} \\ G_{12}=G_{13} & 7.10 & \mathrm{GPa} \\ G_{23} & 3.45 & \mathrm{GPa} \\ v_{12} & 0.30 & \mathrm{GPa} \\ \rho & 1600.00 & \mathrm{~kg} / \mathrm{m}^{3} \\ \sigma_{11 \text { allowable }} & 1447.00 & \mathrm{MPa} \\ \sigma_{22} \text { allowable } & 51.70 & \mathrm{MPa} \\ \tau_{12 \text { allowable }} & 93.00 & \mathrm{MPa}\end{array}$

Balsa Filling

$\begin{array}{lll}E & 530.00 & \mathrm{MPa} \\ v & 0.30 & \\ \rho & 155.39 & \mathrm{~kg} / \mathrm{m}^{3} \\ \sigma_{\text {allowable }} & 13.50 & \mathrm{MPa} \\ \tau_{\text {allowable }} & 7.79 & \mathrm{MPa}\end{array}$




\subsection{Multibody Dynamics Model}

A multibody dynamics model for a baseline design was constructed using DYMORE. The baseline design is XV15 tiltrotor aircraft with three blade gimbaled rotor system for which the main characteristics are given in Table 4-2. Due to symmetry considerations only half of the system is modeled as depicted in Figure 4-2 and Figure 4-3 representing hover and cruise flight conditions, respectively. Rigid bodies are used to model the fuselage and the nacelle while the rotor blades, the wing and the shaft providing torque transmission from the nacelle to the rotor hub are modeled as deformable bodies. The baseline rotor blade stiffness, mass, thickness to chord ratio and built-in twist distributions are shown in Figure 4-4 to Figure 4-7. Various revolute and universal joint connections provide accurate representation of the articulation between the hub and rotor blades as well as the gimball and wing-nacellehub connections. A close-up view of the rotor hub featuring the gimball configuration, a swashplate and the blade hub articulation appears in Figure 4-8.

The aerodynamic loads are calculated along the beam by table lookups for the specified airfoil definitions. The inflow model uses the work of Peter and He [50] which is based on a theory for unsteady flow over a circular disk with a pressure jump across that disk. 
Table 4-2 Baseline System Properties

Wing

Bending stiffness, $E I_{1}$

$10.7 \mathrm{Nm}^{2}$

Bending stiffness, $E I_{2}$

$43.6 \mathrm{Nm}^{2}$

Torsional stiffness, $G J$

$11.5 \mathrm{Nm}^{2}$

Mass

$43.8 \mathrm{~kg} / \mathrm{m}$

Length

$4.902 \mathrm{~m}$

Fuselage mass

$6300 \mathrm{~kg}$

Nacelle mass

$898.8 \mathrm{~kg}$

Rotor Blade

Number of blades

3

Rotor disc radius

$3.81 \mathrm{~m}$

Chord length

$0.355 \mathrm{~m}$

Lift curve slope

6.28

Airfoil distribution

$R<0.277$

64-X25

$0.277<R<0.717$

$64-\mathrm{X} 18$

$0.717<R<0.917$

$64-\mathrm{X} 12$

$R>0.917$

64-X08 


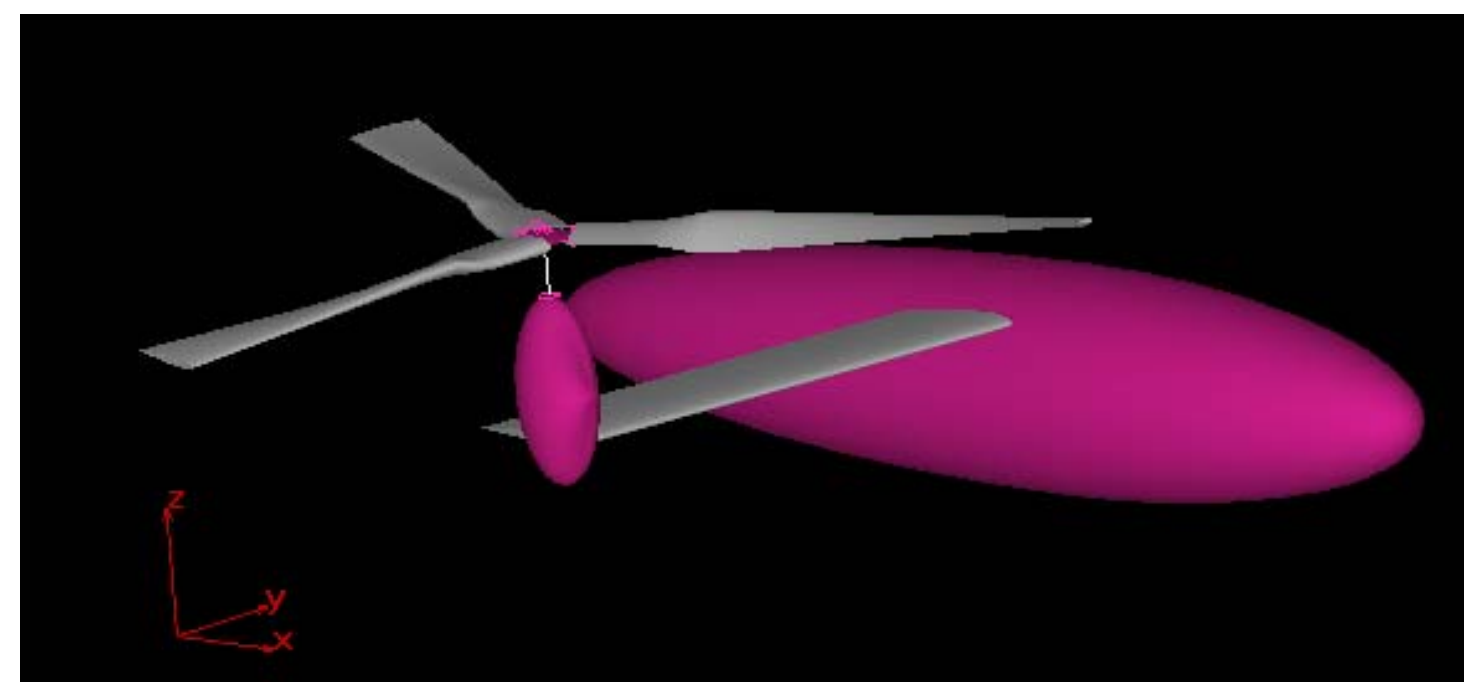

Figure 4-2. XV15 DYMORE Model in Hover Mode

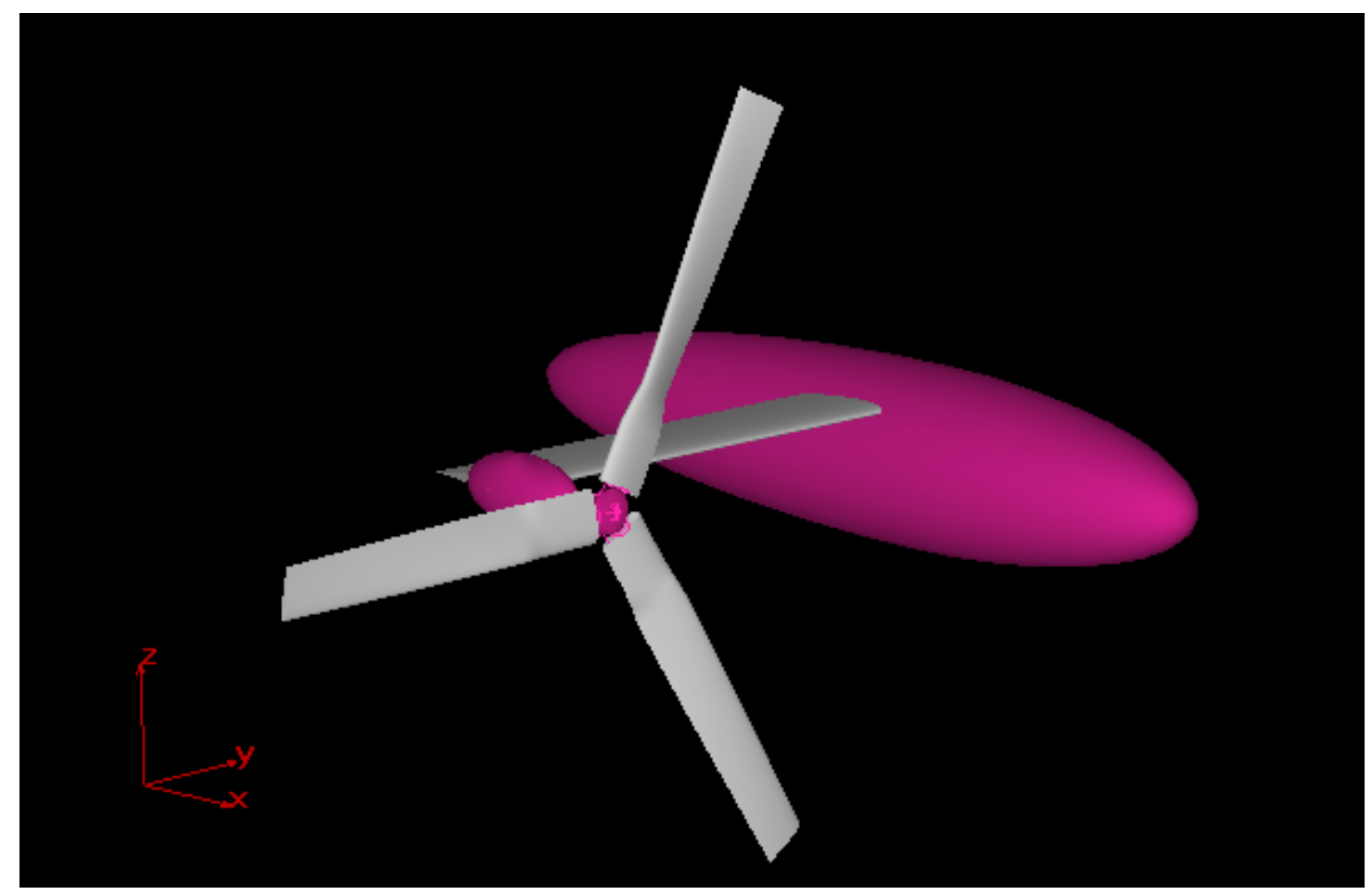

Figure 4-3. XV15 DYMORE Model in Cruise Mode 


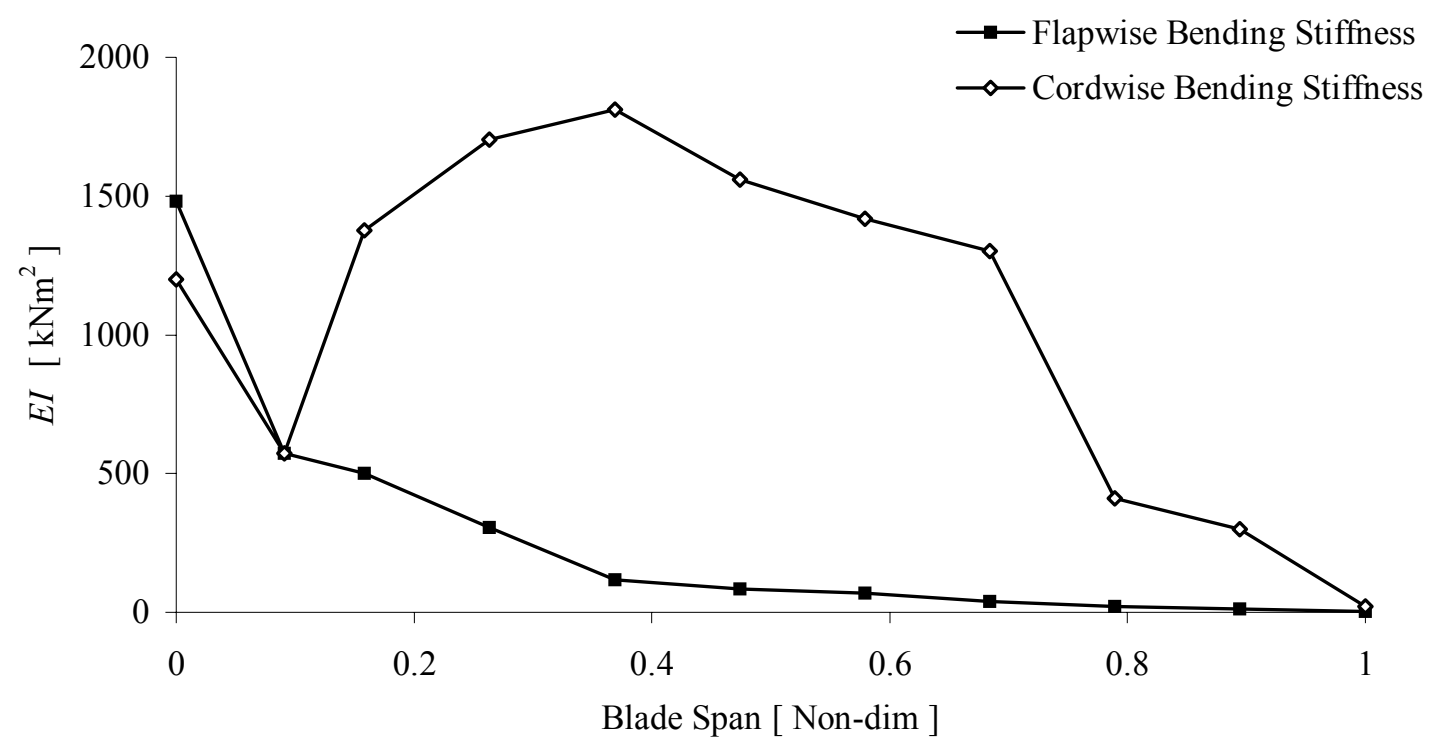

Figure 4-4. Baseline Bending Stiffness Distributions

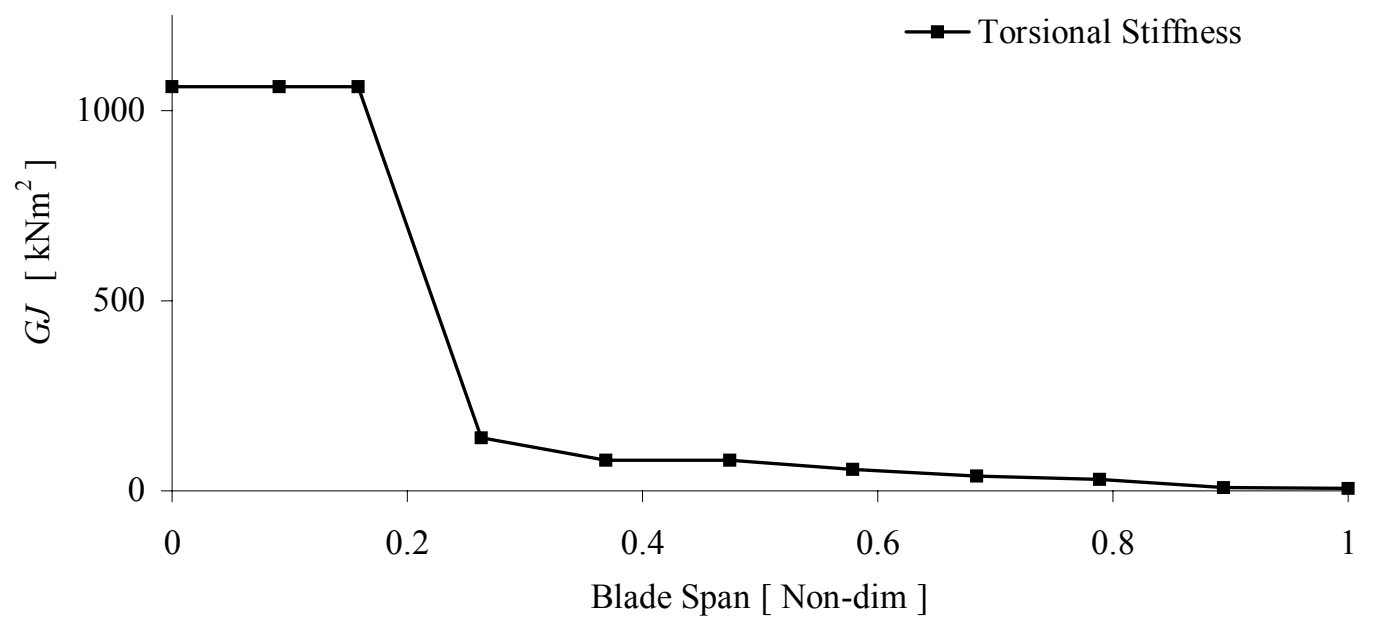

Figure 4-5. Baseline Torsional Stiffness Distribution 


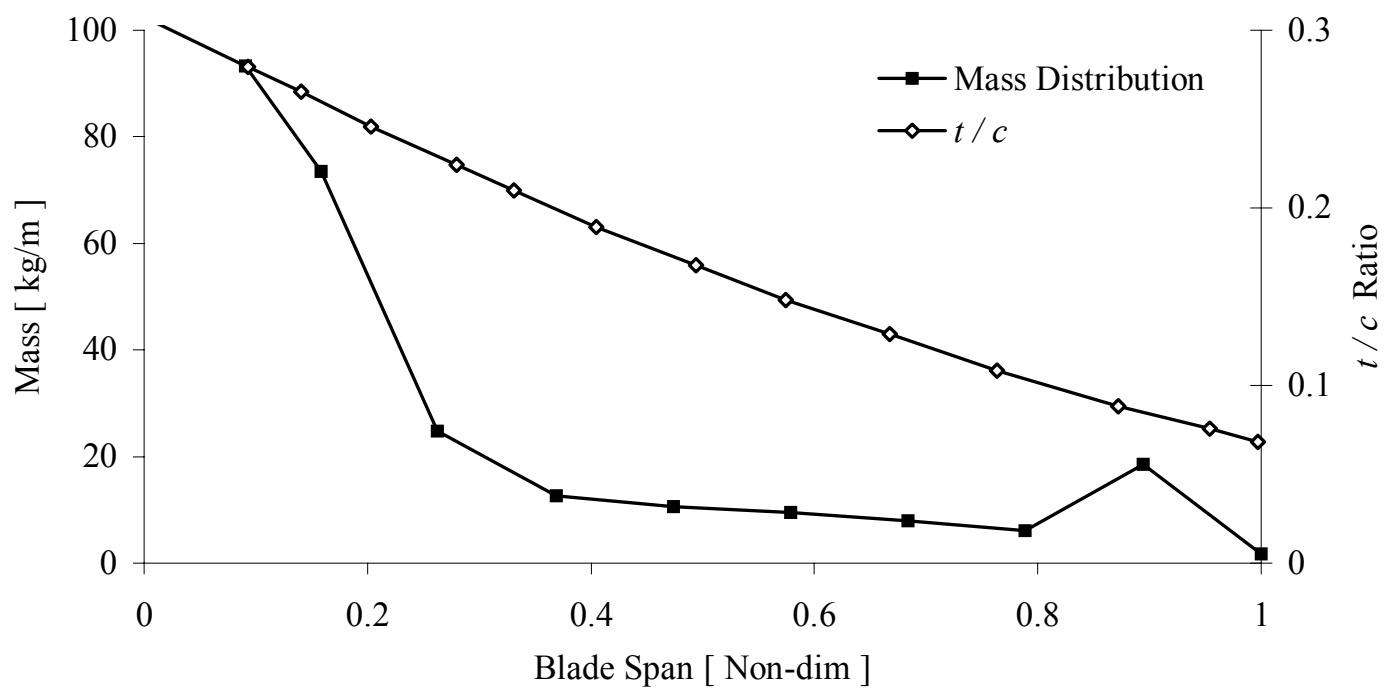

Figure 4-6. Baseline Mass and Thickness/Chord (t/c) Ratio Distribution

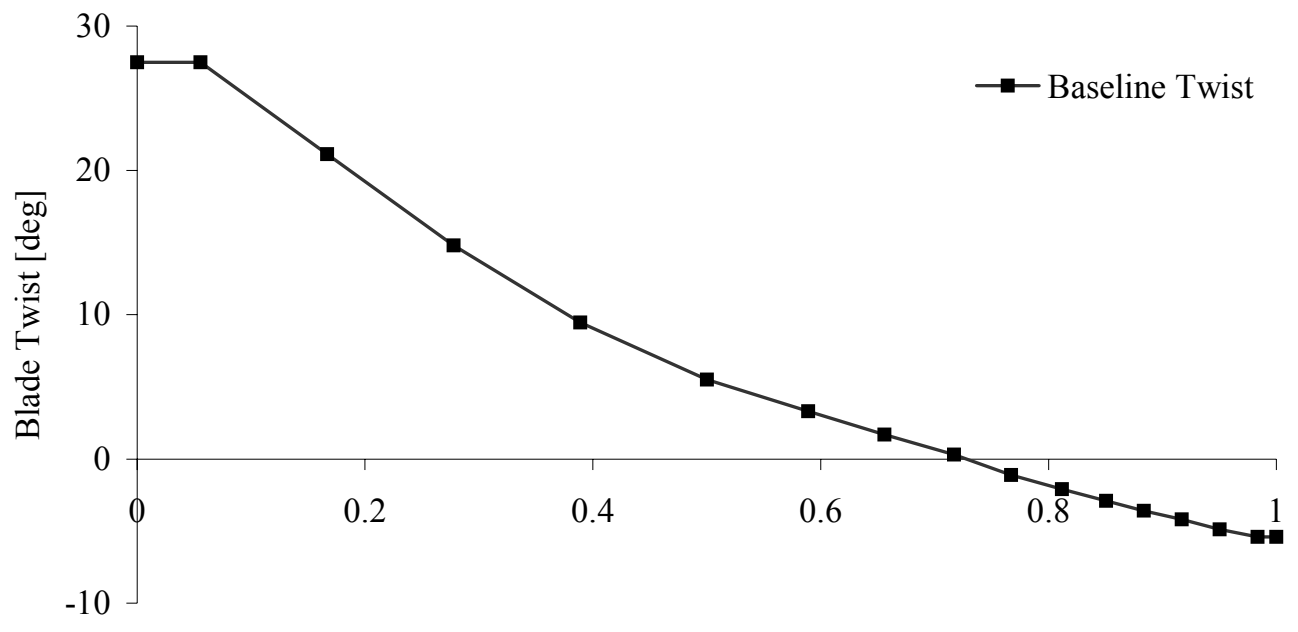

Blade Span [Non-dim]

Figure 4-7. Baseline Twist Distribution 


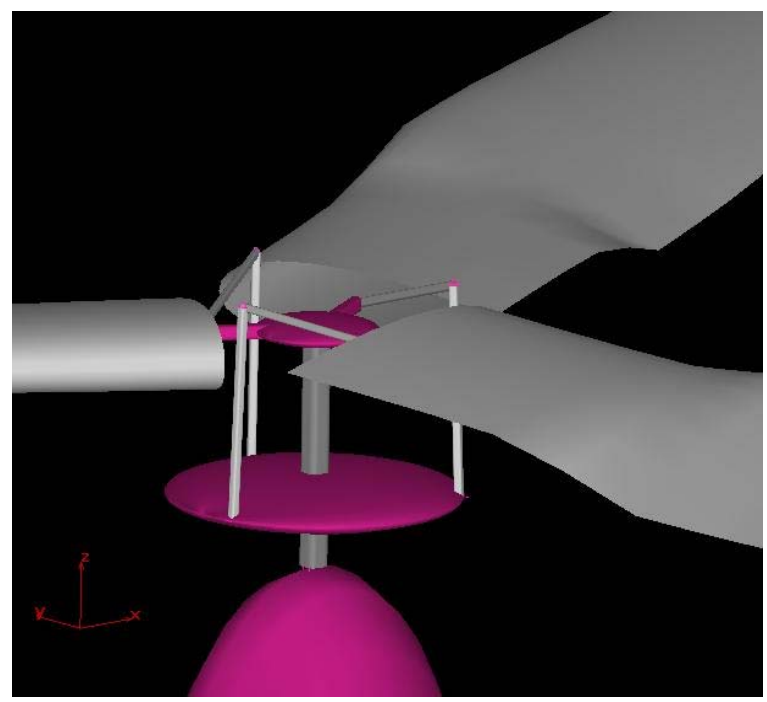

Figure 4-8. Rotor Hub Close-up View

The validation of the baseline design is performed through a power and thrust coefficient comparison with test data [3]. The predictions by the baseline DYMORE model closely matches the test data, as depicted in Figure 4-9.

Figure 4-10 shows the variation of the thrust coefficient with the collective pitch at $R=0.75$. Test data from Ref. [28] were used for comparison. DYMORE results indicated a close correlation with the test data especially for $0.006<C_{T}<0.015$, which covers the range of $C_{T}$ for practical operation conditions. In the performance and thrust calculations the inertial effects are considered in a quasi-static manner and the effects due to the elasticity of the wing are removed by applying clamped boundary condition to the system at the nacelle location. 


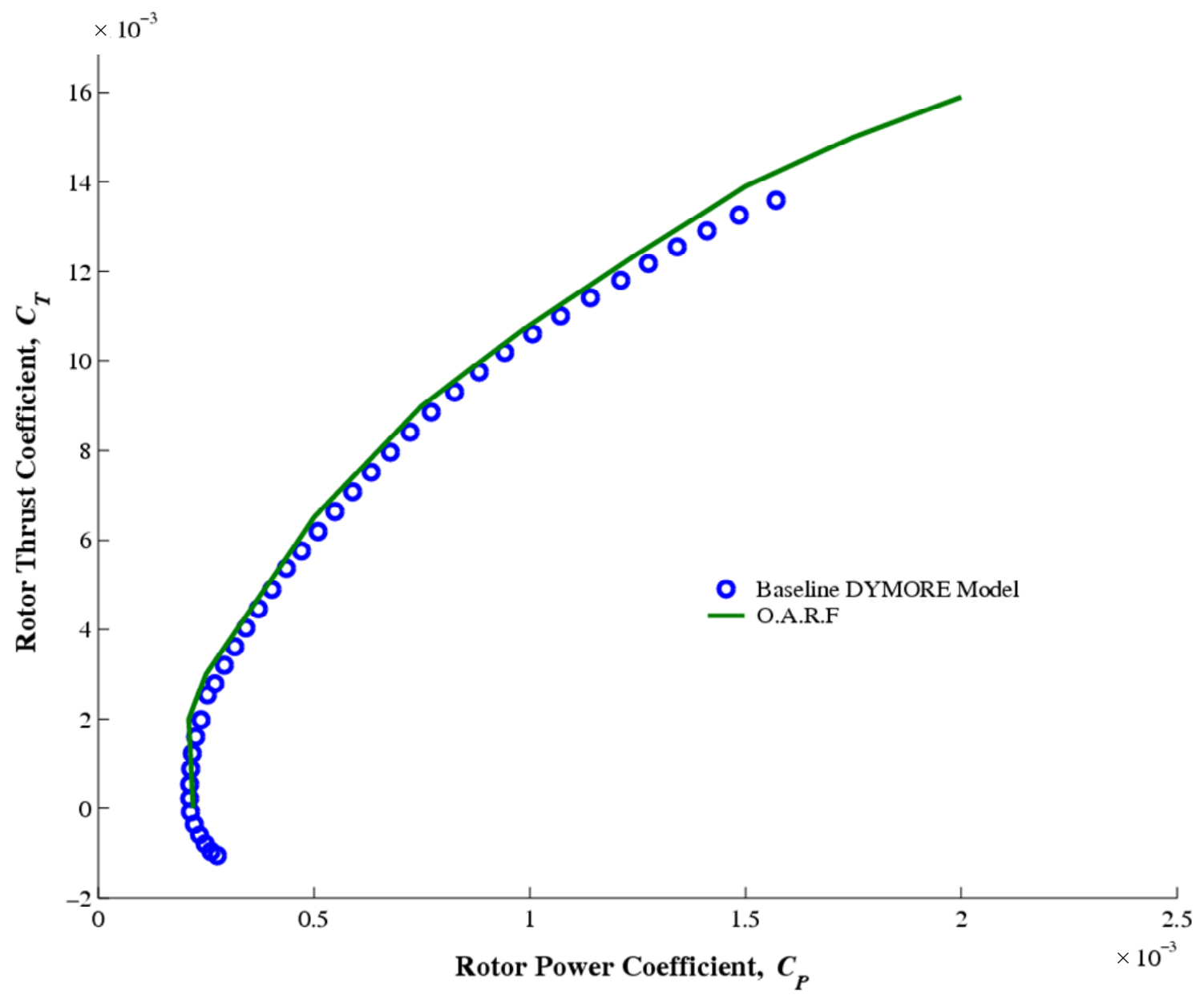

Figure 4-9. Baseline Power vs. Thrust Coefficient Comparison 


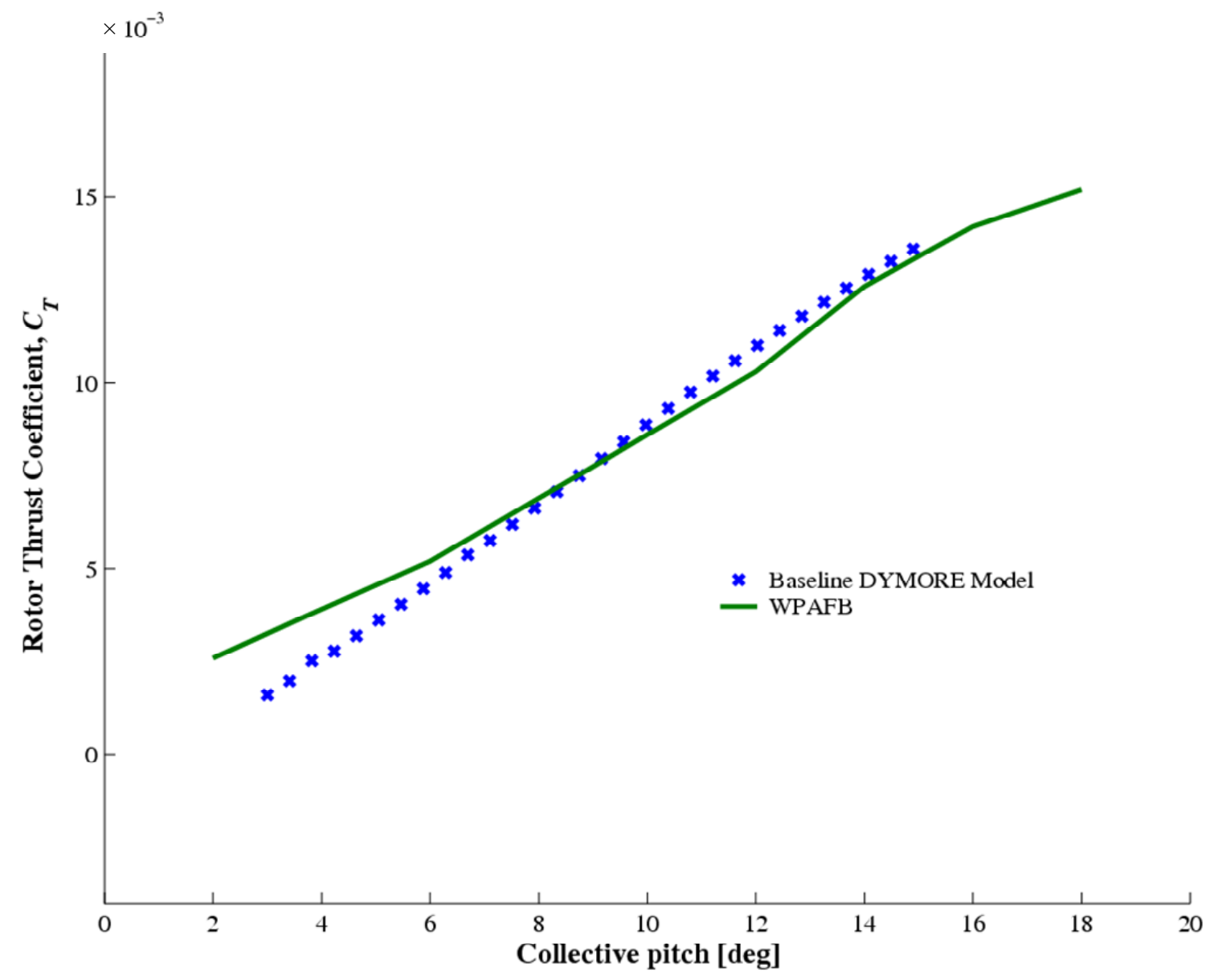

Figure 4-10. Baseline Thrust Coefficient vs. Collective Pitch at $R=0.75$ Comparison

Implementation of the sliding mass concept requires some changes to the baseline DYMORE model. The aim is to provide a means for the leading edge balancing mass to slide with respect to the main rotor housing and shift the axial force due to this mass to the blade tip. A schematic of the modified multibody dynamics model that accommodates such nonstructural mass distribution is illustrated in Figure 4-11. 


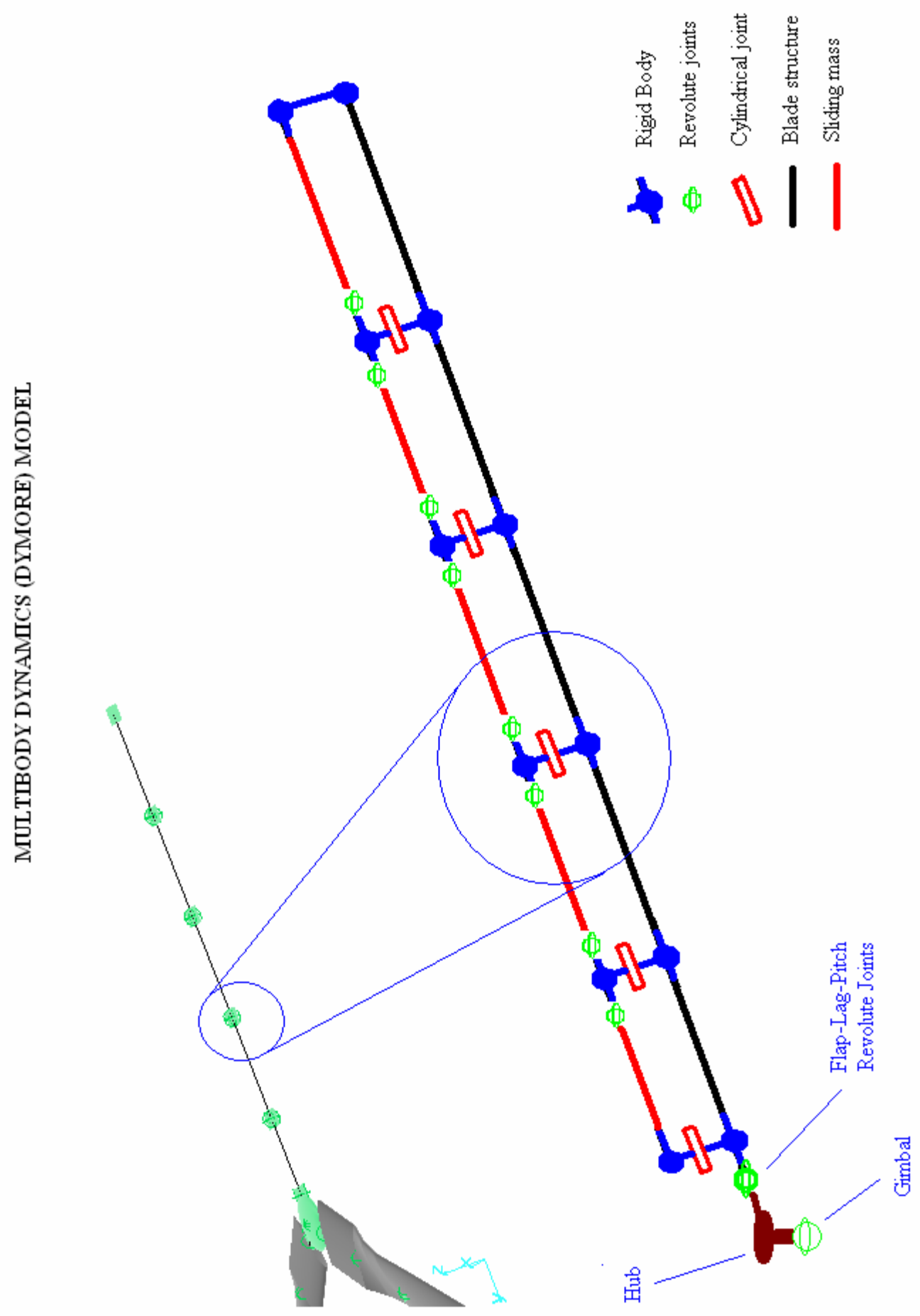

Figure 4-11. DYMORE Model for Sliding Mass Concept 
The black solid line in the figure represents the main rotor blade load-carrying member. It is connected to the rotor hub through a set of revolute joints representing the articulation between the hub and the blade. The rigid bodies illustrated by the blue dots are specific to DYMORE modeling and provide a means to connect various elements without altering the mass and stiffness properties of the system. The five piece green line symbolizes the sliding mass. The pieces are connected to each other through a set of revolute joints. The cylindrical joints provide the connection between the sliding mass and the blade structure at the same location. Cylindrical joints are characterized by two degrees of freedom, a rotation and a displacement about the cylinder axis. This configuration assures that the sliding mass is free to move with respect to the main rotor structure and the elements representing the sliding mass do not contribute to the stiffness of the blade. Finally the sliding mass is connected to the blade with a rigid connection at the blade tip providing the path for the axial load continuity onto the main structure at the blade tip.

The configuration leaves the elements representing the sliding mass under high compressive loads. Additionally the pinned-pinned end conditions associated with the revolute joints at each end of these elements make them prone to buckling instability. This causes divergence problems in the DYMORE simulations. To overcome this difficulty, high bending stiffness values have been chosen to characterize the sliding mass elements. This also brings about the importance of designing the model in such a way that it will not contribute to the overall stiffness of the blade elements.

One concern with the sliding mass concept is whether the load can still be effectively transferred when the blade is deformed. The answer to this question depends on how the concept is incorporated in practice. However, the model does take into account such concerns 
since the cylindrical joint axis will not always be normal to the rotational velocity vector. There will be small but finite value of axial load from the sliding mass onto the rotor blade itself in each cylindrical joint location.

The effect of the sliding mass model on the baseline design can be evaluated by comparing the rotor blade frequencies, as the frequencies are directly related to the stiffness and mass characteristics of the structure. Non-rotating blade frequencies for the baseline and baseline modified for the sliding mass concept are presented in Table 4-3. The stiffness and mass distributions for both designs are identical. The only difference is in the mass left to slide, $M_{S}$, which is assumed to be $0.5 \mathrm{~kg} / \mathrm{m}$ for the modified model. The blade frequencies were obtained using a model that consists of the blade, articulation, hub and the gimball, which was clamped onto the inertial frame. All the coupled flap, lag and torsion frequencies match within $1.3 \%$, proving that the modified model does not alter the stiffness of the original baseline design.

Table 4-3 Non-rotating Collective Mode Blade Frequencies

\begin{tabular}{ccc} 
& Baseline $[\mathrm{Hz}]$ & Baseline with $M_{S}=0.5 \mathrm{~kg} / \mathrm{m}[\mathrm{Hz}]$ \\
\hline F1 & 2.7 & 2.7 \\
L1 & 6.9 & 6.9 \\
F2/L2 & 14.3 & 14.3 \\
F3/L2 & 40.3 & 40.5 \\
T1 & 63.9 & 63.1
\end{tabular}


The variations of the baseline frequencies with the rotor speed in hover are depicted in Figure 4-12. Again both systems have equal stiffness and mass distributions while the modified model has $M_{S}=0.5 \mathrm{~kg} / \mathrm{m}$. The figure reveals that the variation of the natural frequencies with rotor speed shows similar behavior in both modified and unmodified baseline models. The model with the sliding mass tends to have higher frequencies and this becomes more evident at the higher levels. The increase in frequency with rotor speed is attributed to the stiffening effect due to the inertial forces. Since the aim of the sliding mass is to shift the centrifugal force to the blade tip, it is not surprising for the model with the sliding mass to reach higher frequency levels than the baseline as the stiffening effect is expected to be more pronounced. 


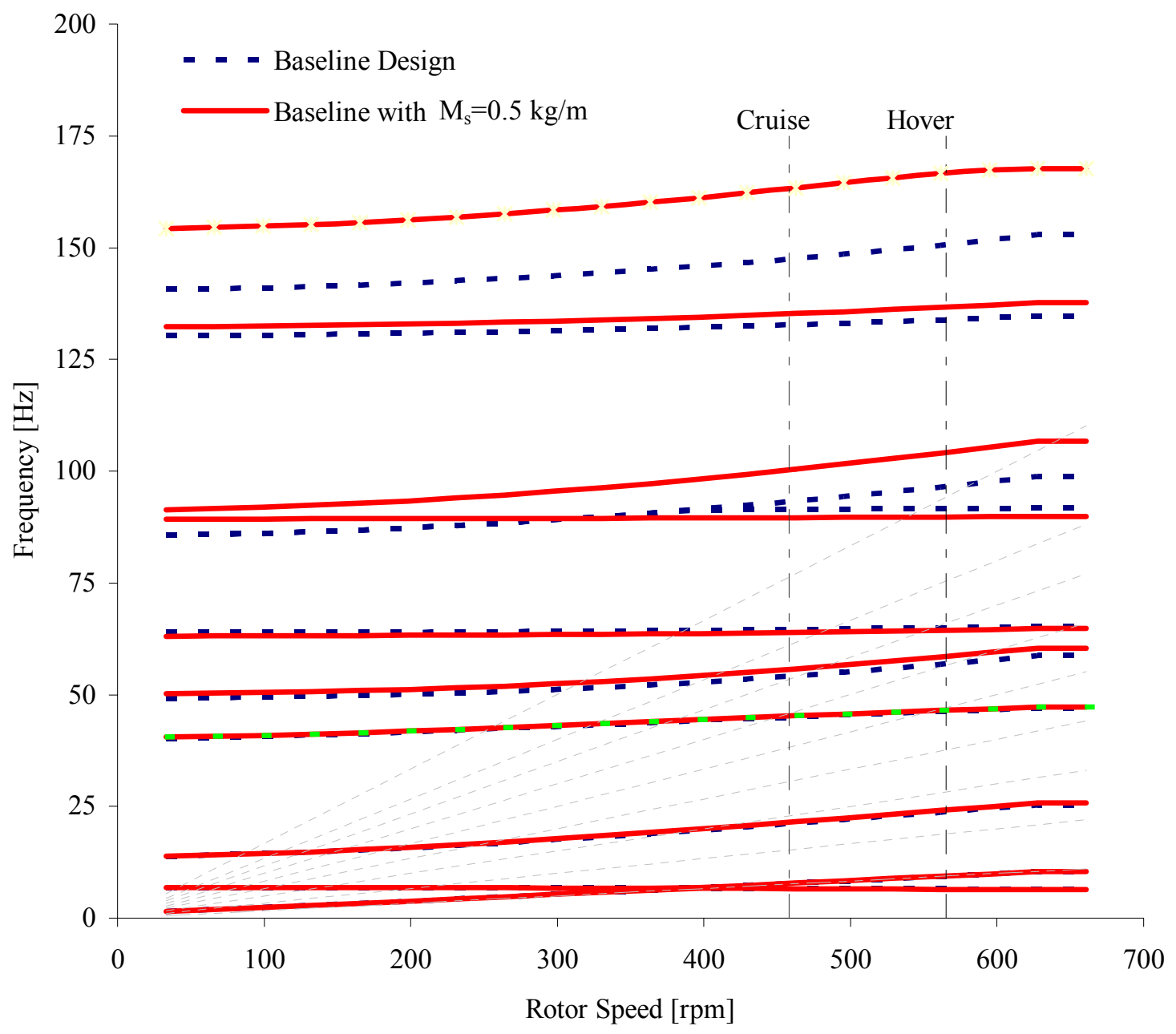

Figure 4-12. Frequency vs. Rotor Speed 


\subsection{Optimization Implementation}

\subsubsection{Design Objective}

In order to set target performance improvement figure it is necessary to study the effect of the twist rate on the baseline design performance. This is carried out by generating power versus twist rate curves in the two operating modes. Taking into account the conflicting behavior between the torsional stiffness and extension-twist coupling it is reasonable to state that only the outer regions of the blade can be effectively tailored for extension-twist coupling. Hence considering the twist rates for $R>0.5$ would be a better way to investigate the relationship between twist rates versus power requirements.

The baseline blade twist distribution can be accurately represented by a linear function with a twist rate of $23 \mathrm{deg} / R$ for $R>0.5$. Figure 4-13 illustrates the rotor blade twist distributions for different twist rates including the baseline design. For each of these twist distributions power requirement in hover and cruise modes are calculated. The hover results are depicted in Figure 4-14. The vertical axis in the figure represents the percent change in power requirement with respect to the baseline design for a thrust coefficient of 0.010 . It should be noted that the curve crosses the twist rate axis at $23 \mathrm{deg} / R$, referring to the baseline design condition. As a negative change represents an improvement, it can be seen that a twist rate of about $12 \mathrm{deg} / R$ would result in about 5 to $6 \%$ improvement in power requirement in hover for $C_{T}=0.010$. 


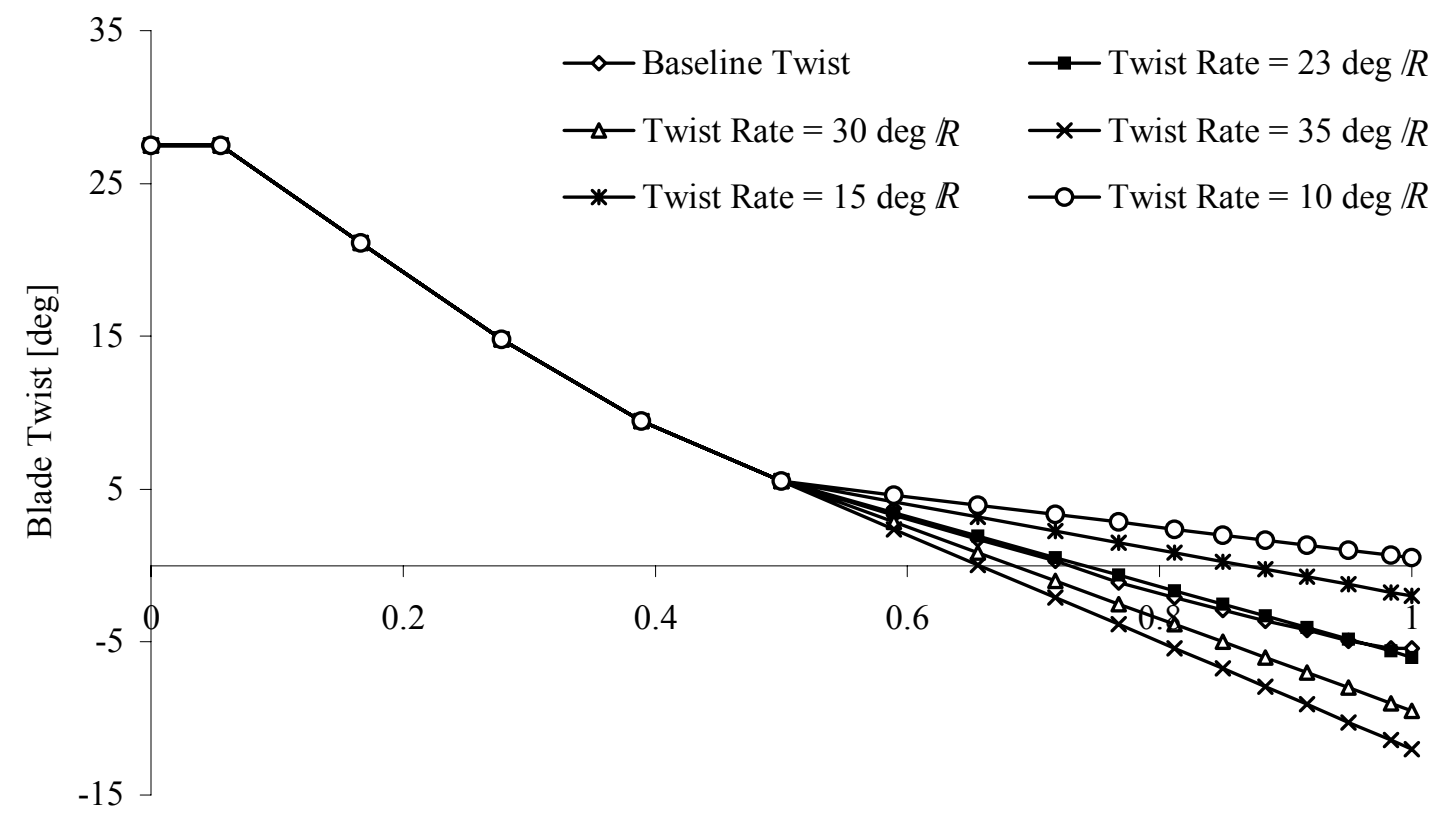

Blade Span [Non-dim]

Figure 4-13. Twist Rate Distributions

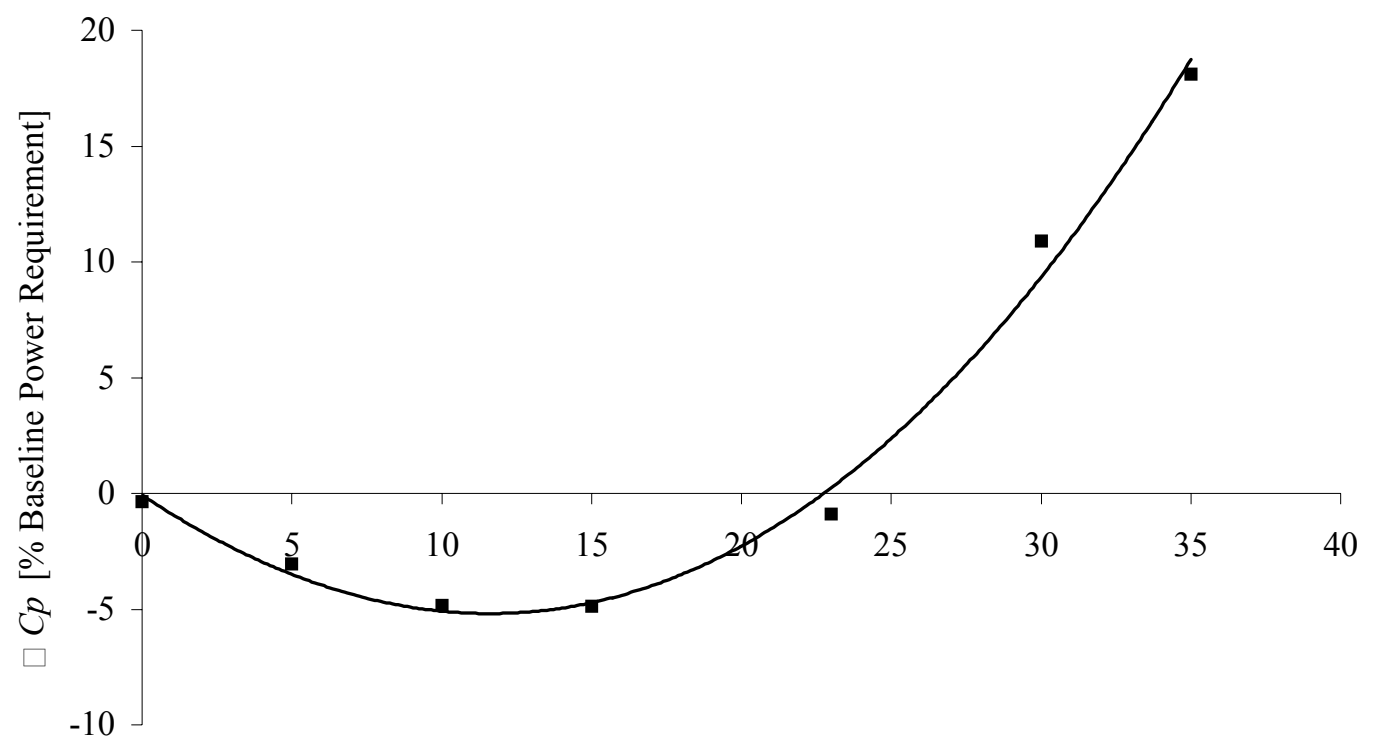

Twist Rate $[\operatorname{deg} / R$ ]

Figure 4-14. Change in Power Requirement in Hover at $C_{T}=0.01$ vs. Twist Rate 
The effect of the twist rate on the power requirement in the cruise mode is demonstrated in Figure 4-15. The plot shows that very high twist rates would be required for an improvement in the cruise mode and the improvement would be limited to about $1 \%$. Small improvement envelope in this mode might be related to the fact that the baseline system was already optimized for the cruise mode, since the vehicle operates mostly at this flight condition. The amount of passive twist achieved through elastic tailoring is very limited. Hence the twist rate for the cruise mode will be left as the baseline twist rate and the improvement in hover mode will be sought after.

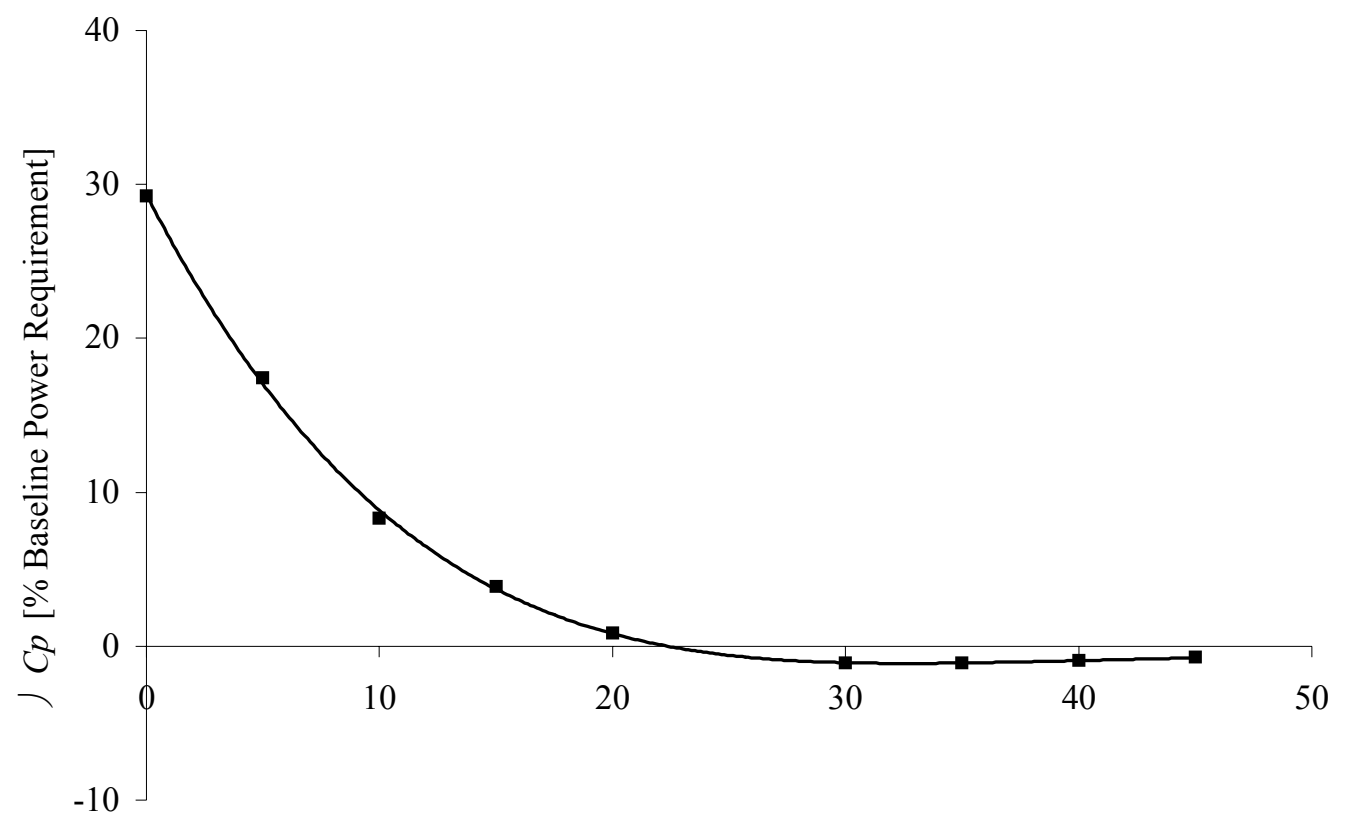

Twist Rate [ $\operatorname{deg} / R$ ]

Figure 4-15. Change in Power Requirement in Cruise at $C_{T}=0.01$ vs. Twist Rate 
Table 4-4 summarizes the design objective for this work. The design targets a twist rate of $12 \mathrm{deg} / R$ in hover and about 5 to $6 \%$ improvement in hover power requirement is predicted for this twist rate. In the cruise mode the baseline twist distribution and performance figures will be matched.

Table 4-4 Design Objectives

Twist rate for $R>0.5,[\mathrm{deg} / R]$ Performance Improvement

$\begin{array}{llc}\text { Hover } & 12 & 5 \text { to } 6 \% \\ \text { Cruise } & 23 & \text { Baseline Figures }\end{array}$

\subsubsection{Optimization Process}

A two-level design optimization process is applied to achieve performance improvement in hover. In the lower level optimization, the system is considered as a whole and optimum coupling distribution and sliding mass values that would give the desired blade twist rate are determined. These optimum values are later used in the higher level optimization as target values in a relatively detailed blade cross-sectional sizing process. A flowchart of the optimization procedure is given in Figure 4-16.

In the lower level optimization a simple box-beam cross-sectional model and a single blade multibody dynamics model are implemented. The goal of this step is to determine the required extension-twist coupling values for an optimum system. Rather than using the stiffness values as design variables directly, the box-beam model is utilized to generate positive definite and feasible stiffness values that can readily and easily be used by the 
multibody dynamics model in a cost effective way. The box-beam model provides the blade stiffness parameters for a given set of design inputs including the cross-sectional geometric parameters and the composite layup. As discussed previously only the outer board of the blade, $R>0.5$, is considered for elastic tailoring. The elastic properties are calculated at five equally spaced locations along the span of the beam for $R>0.5$. The geometric parameters of the model are bounded such that the baseline design thickness to chord ratio is maintained.

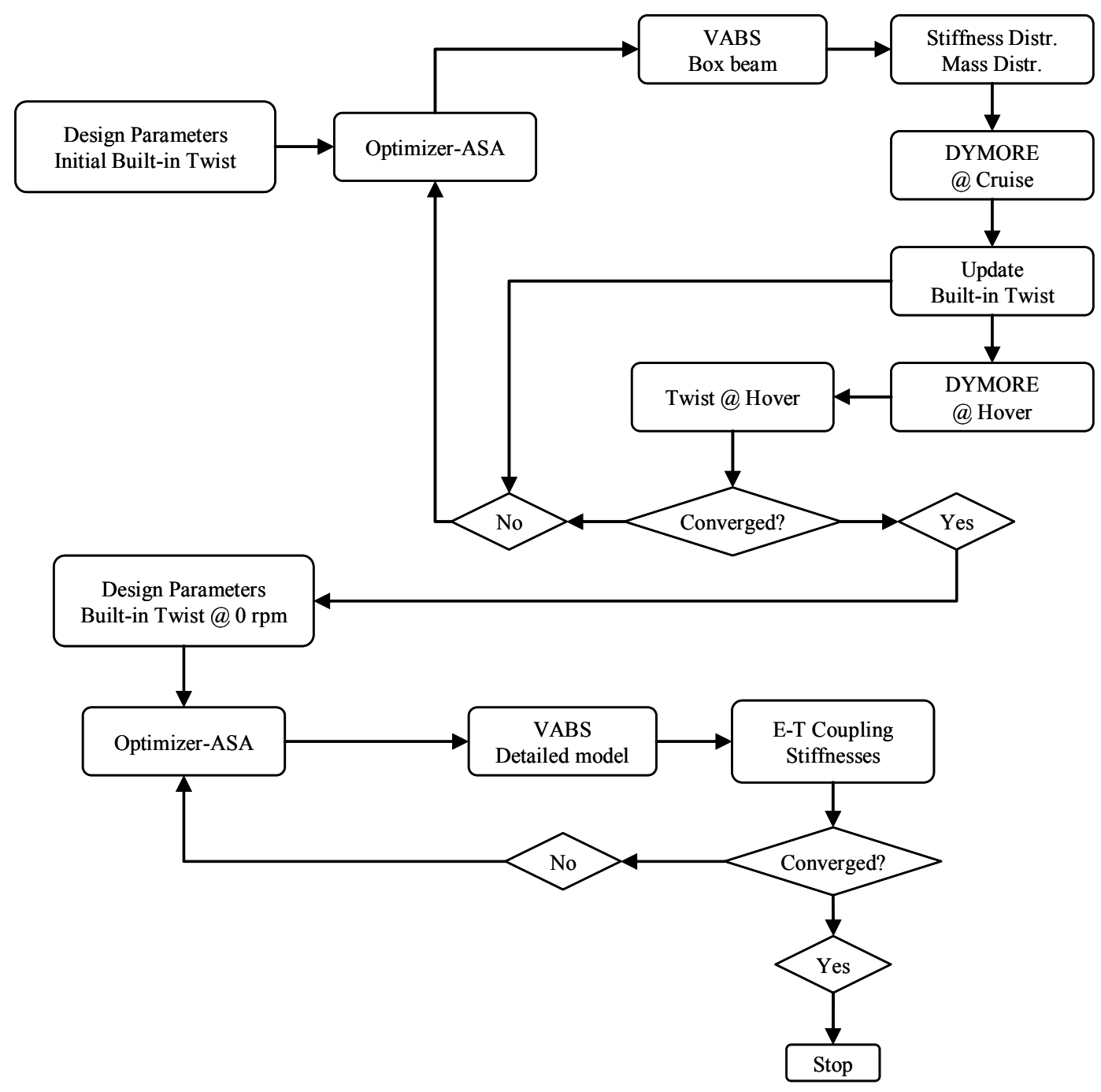

Figure 4-16. Multilevel Optimization Process 
The blade torsional stiffness distribution is also constrained to be equal or larger than the baseline design such that a realistic stiffness matrix can be transferred to the multibody dynamics model. The inertial properties of the system are taken as the baseline design configuration. The composite layup system is chosen to be circumferentially uniform with two ply angles. An illustration of the cross-sectional model showing the design parameters is depicted in Figure 4-17.

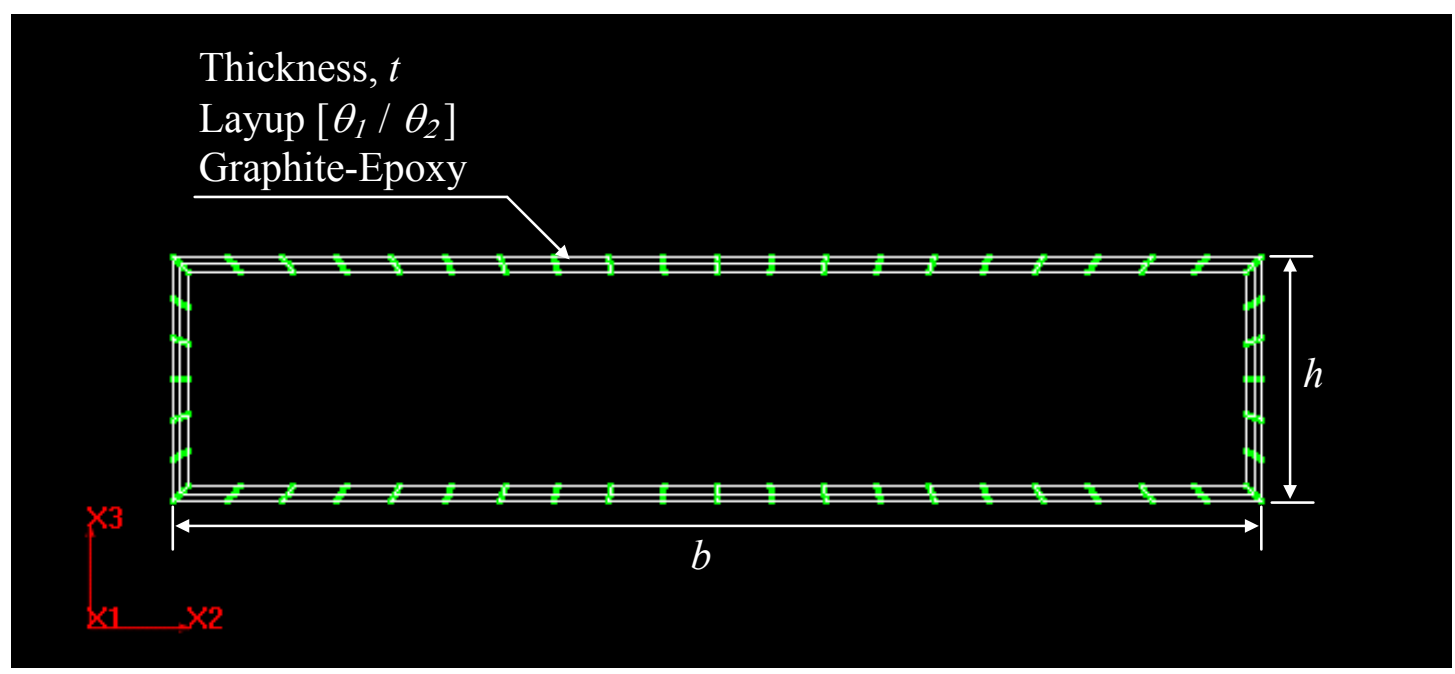

Figure 4-17. Composite Box Beam Model

The stiffness matrix obtained through the cross-sectional analysis is then transferred into DYMORE for a static blade analysis. A screenshot for the DYMORE model is shown in Figure 4-18. The system is modeled as a single blade rotor with blade hub articulation and a clamped hub configuration. The airloads, gravity and centrifugal loads are applied on the system quasi-statically. In the first step of the analysis the rotor speed is taken as the cruise 
speed of $48 \mathrm{rad} / \mathrm{s}$. Using the twisting deformation at this speed, the blade built-in twist rate is adjusted such that the system attains the baseline built-in twist rate distribution at the cruising speed. Later the system is rerun using the updated built-in twist at the hovering condition with rotor angular speed of $59.2 \mathrm{rad} / \mathrm{s}$. The final twist distribution is compared to the previously discussed optimum twist distribution with $12 \mathrm{deg} / R$ twist rate. This process is repeated as needed by the optimizer until convergence within $0.01 \%$ or the maximum of $10^{5}$ design inputs have been achieved.

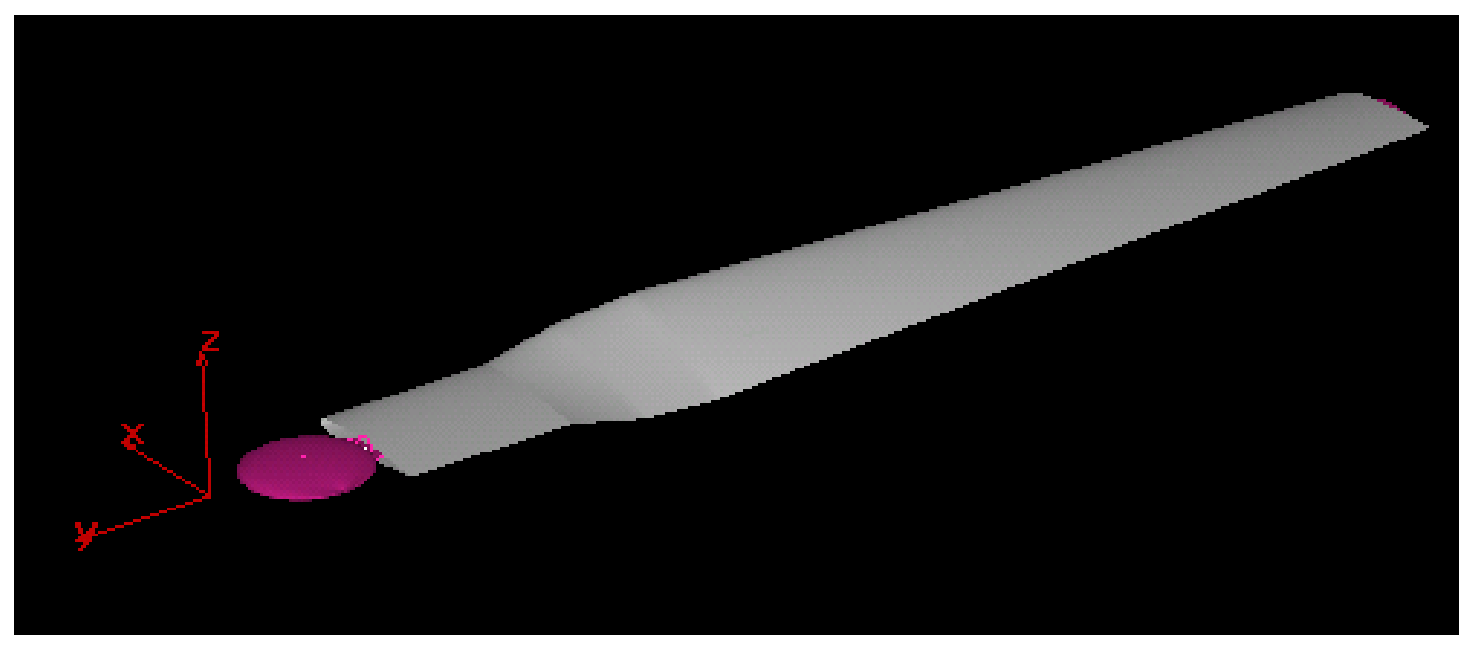

Figure 4-18. Lower Level Optimization DYMORE Model 
The overall lower-level optimization problem can be summarized as,

$$
\begin{aligned}
& \text { Find }\left(h_{i}, b_{i}, \theta_{1}, \theta_{2}, M_{S}\right) \text { or, }\left(E T_{i}, M_{S}\right), \quad i=1 . .5 \\
& \text { For } \min \left|\phi_{\text {baseline }}-\phi_{\text {model }}\right| \\
& \qquad \begin{array}{l}
t / c=(t / c)_{\text {baseline }} \\
\text { Subject to } \quad G J \geq G J_{\text {baseline }}
\end{array}
\end{aligned}
$$

In the upper level optimization the extension-twist coupling values determined by the lower level optimizer are used as design targets for a cross-sectional sizing process. The design variables at each span location included the D-Spar web location $\left(r_{s}\right)$ and thickness $\left(t_{2}\right)$, the skin thickness $\left(t_{1}\right)$ and the ply angles used for the composite skin $\left[\theta_{1} / \theta_{2}\right]$ and D$\operatorname{spar}\left[\theta_{3} / \theta_{4}\right]$, as depicted in Figure 4-19.

It has been shown in [48] that an angle ply stacking sequence does not provide optimum extension-twist coupling for composite strips. It was proved that the maximum available extension-twist coupling follows an asymptotic behavior with respect to the number of plies used in the stacking sequence and at least two different ply angles are required to maximize the tension-torsion effect. However the asymptotic value is reached rapidly and changing to four plies does not yield a significant increase in the available extension-twist coupling. Based on this observation the stacking sequence is chosen to be circumferentially uniform with $\left[\theta_{1} / \theta_{2}\right]$ for the airfoil skin and $\left[\theta_{3} / \theta_{4}\right]$ for the spar in order to reduce computational cost. 


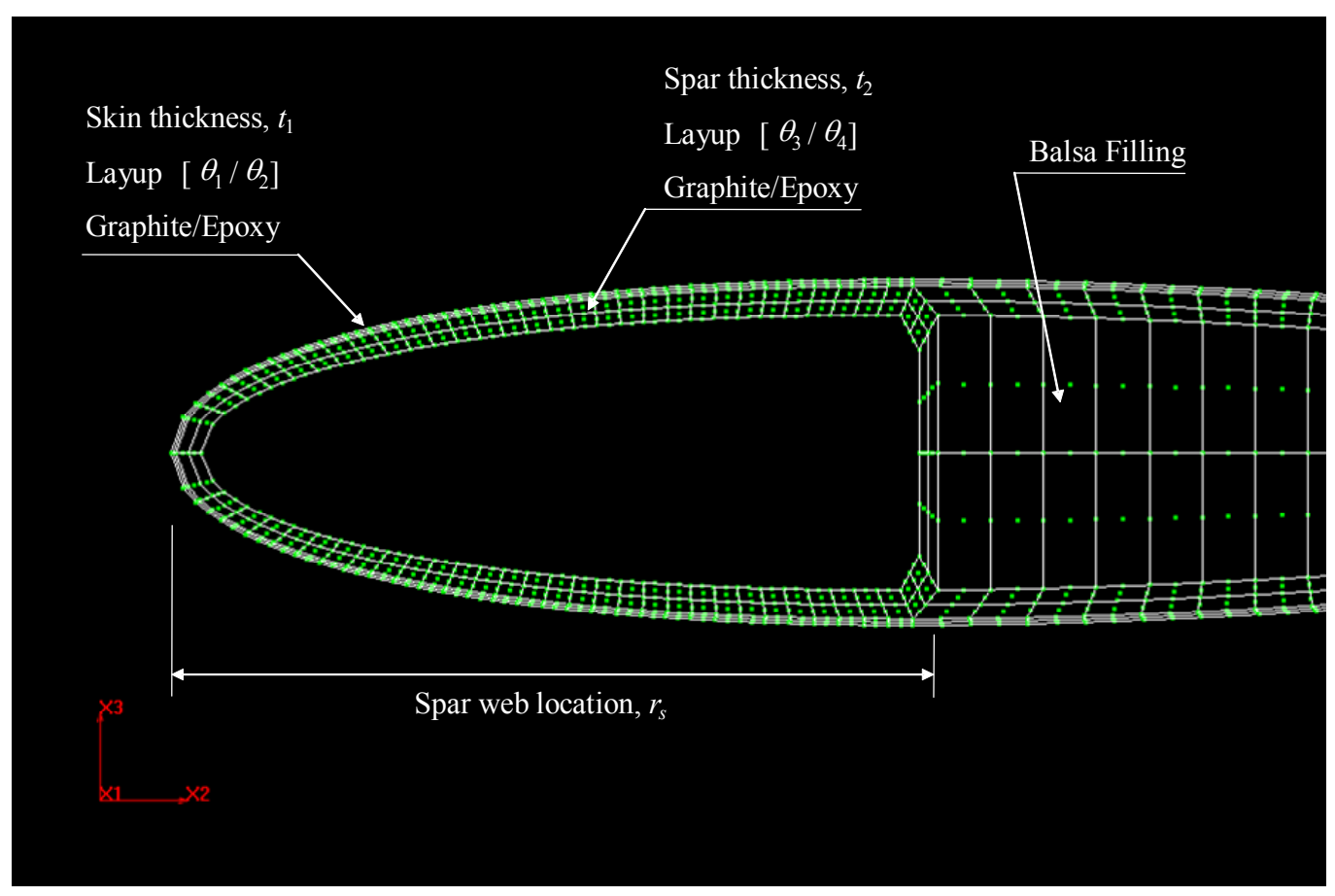

Figure 4-19. Upper Level Optimization Cross-sectional Model

To meet manufacturability requirements the skin and spar thicknesses are chosen as multiples of ply thickness and the composite layup is chosen to be constant over the blade span with angles chosen as multiples of 5 degrees. An investigation of a case where the constant ply angle constraint is relaxed can be found in the Appendix. Also cases where hygrothermal effects are eliminated, such as stacking sequences based on Winckler [64] system, are considered. For that purpose a hygrothermally stable model with $\left[\theta /\left(\theta+90^{\circ}\right)_{2} / \theta\right]$ type stacking sequences is investigated and the results are presented in the Appendix.

The stiffness properties of beams changes with built-in twist rate. However, the builtin twist rate is defined based on the final achieved twist distribution, which is not known 
prior the optimization. For this reason the effect of the built-in twist on the stiffness properties is reflected on the model by using an average twist rate, chosen to be the built-in non-rotating twist rate distribution calculated in the lower-level optimization process.

The design objective for this optimization step is to achieve the desired extensiontwist coupling values provided by the lower level optimizer subject to constraints on the geometry, stiffness and strength of the system. The thickness to chord ratio is matched to the baseline design using bounding constraints on the geometric parameters. The constraints on the stiffness properties of the system are reflected in the objective function. The torsional stiffness together with the chordwise and flapwise bending stiffnesses will be matched to the baseline design variables as close as possible. The strength of the system under prescribed static loading is checked by a Tsai-Wu failure criteria applied on the stress values measured by 72 sensors placed strategically over the blade cross-section. The steady state baseline loads determined at the hovering condition with $1 \mathrm{~kg} / \mathrm{m}$ sliding mass, depicted in Figure $4-20$, are chosen as the loading condition. They are applied on the quarter chord location. The associated coordinate system for the loading is illustrated in Figure 4-21. 


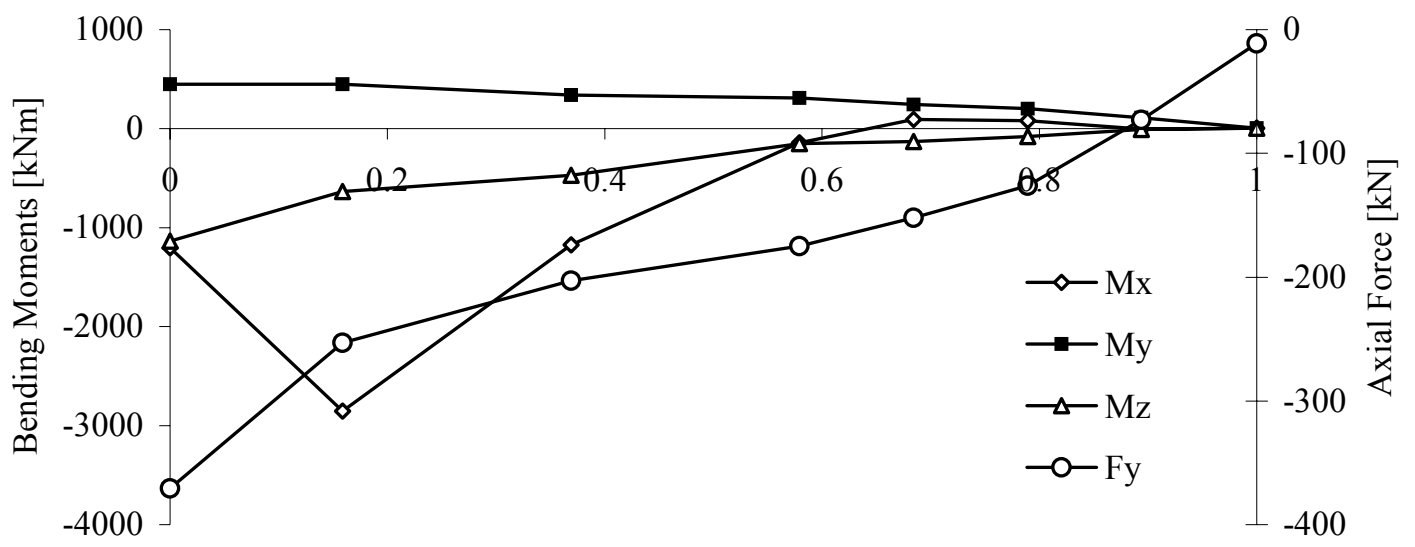

Blade Span [Non-dim]

Figure 4-20. Baseline Steady State Loads

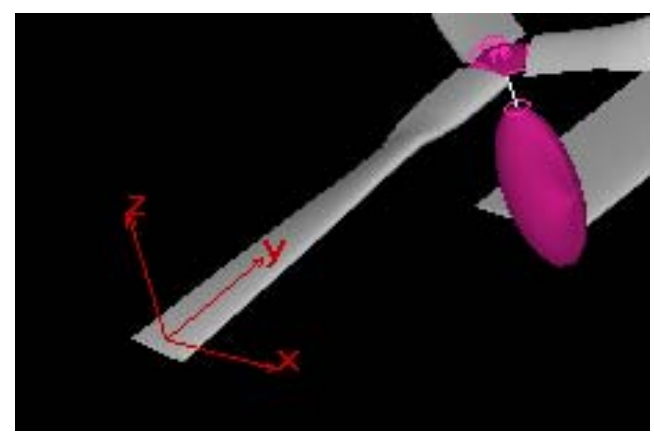

Figure 4-21. Blade Loading Coordinate System

The design problem for the upper level optimization can be stated a follows, 
Find $\left(\theta_{1}, \theta_{2}, \theta_{3}, \theta_{4}, t_{1, i}, t_{2, i}, r_{s, i}\right), \quad i=1 . .5$

$$
\begin{aligned}
& \text { For min }|| E T_{\text {optimum }, i}-E T_{i}|| G J_{\text {baseline }, i}-G J_{i}|| E I f_{\text {baseline, } i}-E I f_{i}|| E I c_{\text {baseline }, i}-E I c_{i}|| \\
& \text { Subject to } t / c=(t / c)_{\text {baseline }}
\end{aligned}
$$

An optimizer based on the Simulated Annealing (SA) method is utilized for optimization. The SA technique imitates mathematically the annealing of metals and formation of crystals. It is a probabilistic design methodology that can deal with discrete or integer variables and has an increased likelihood of finding global minimum for problems having local minima. For the current optimization the design space is formed such that the variables for the ply angle and the skin/spar thickness are discrete values, as the ply angles are multiples of 5 deg and the thickness are multiples of the laminate ply thickness. At this point the ability of SA techniques to deal with discrete variables is very beneficial. The SA algorithm is composed of the following steps [19].

1. Start with a current design $X$, with objective function $F(X)$

2. Perturb the current design resulting in $X_{P}$

3. If $F\left(X_{P}\right) \leq F(X)$ then $X_{P}$ is the current design. 
4. If $F\left(X_{P}\right)>F(X)$ then $X_{P}$ is a worse design, but accepted if a random number $P$, with $0 \leq P \leq 1$, "drawn" for this design is greater than an "acceptance probability" $P_{a c c}$ defined as:

$$
P_{a c c}=\exp (-1 / T)
$$

5. Repeat until convergence while $T$ is reduced as the algorithm proceeds

Accepting the design $X_{P}$ even when it is a worse design causes the algorithm to jump out of local minimum and increases the probability of achieving a global optimum solution. One drawback of the SA technique is its speed compared to other minimization routines. SA is computationally expensive especially if the cost function itself is expensive to compute. However, the discrete design inputs shrink the design space considerably and help reduce the overall cost. Since the scope of this research is not focused on developing an optimization technique for rotorcraft applications and given enough computational power, it is justifiable to use this technique as it increases the likelihood of achieving global optimum.

The optimization in this research is performed using the Adaptive Simulated Annealing code ASA [34]. A Matlab ${ }^{\circledR}$ gateway code ASAMIN [55] has been utilized to facilitate interface among VABS, DYMORE and ASA. 


\section{CHAPTER 5}

\section{Optimization Results}

\subsection{Lower Level Optimization}

In the lower level optimization the target extension-twist coupling distribution and the associated constant sliding mass value required to match the proposed optimum blade twist distribution at the hovering condition is determined. The optimum twist distributions at the hovering and cruise modes are shown in Figure 5-1. The cruise mode twist distribution is taken as the baseline built-in twist distribution as discussed previously.

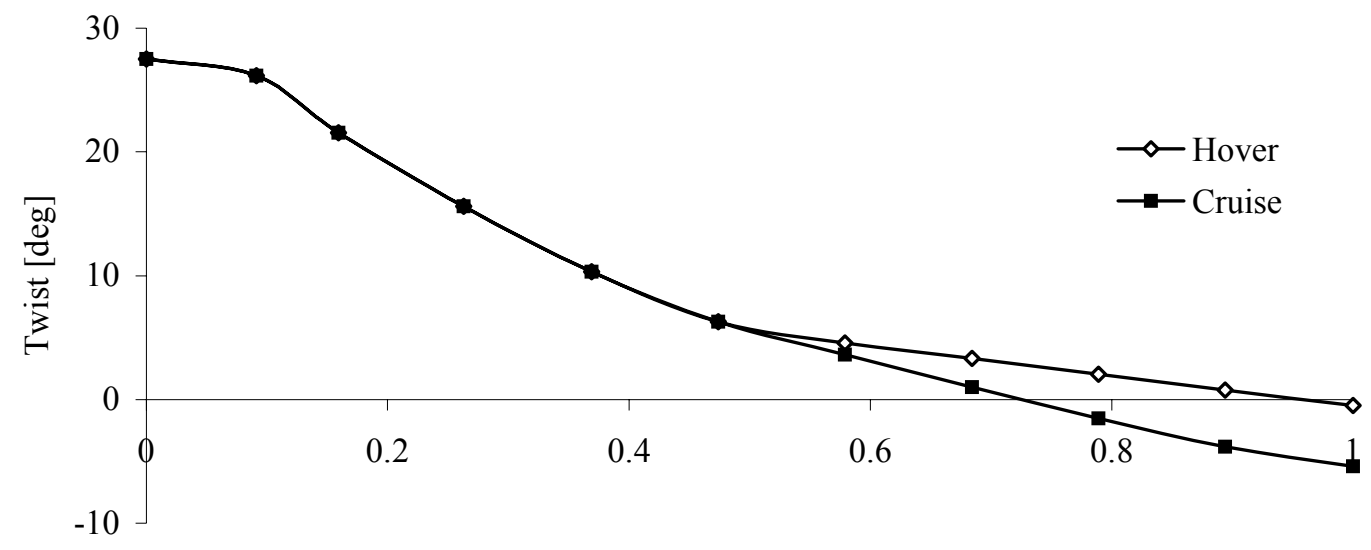

Blade Span [Non-dim]

Figure 5-1. Optimum Blade Twist Distributions 
The elastic properties are calculated at five equally spaced locations along the span of the beam for $R>0.5$. The optimum design parameters for the system are tabulated in Table $5-1$.

Table 5-1 Optimum Lower-Level Design Parameters

\begin{tabular}{|c|c|c|c|}
\hline \multicolumn{2}{|c|}{ Sliding Mass $[\mathrm{kg} / \mathrm{m}]$} & & \\
\hline 0.997297 & \multirow[b]{2}{*}{$\theta_{2}[\mathrm{deg}]$} & & \\
\hline$\theta_{1}[\mathrm{deg}]$ & & & \\
\hline 88.4 & 38.5 & & \\
\hline Span [non-dim] & Width [m] & Height [m] & Skin thickness [mm] \\
\hline 0.58 & 0.213 & 0.105 & 1.000 \\
\hline 0.68 & 0.196 & 0.089 & 1.007 \\
\hline 0.79 & 0.210 & 0.074 & 1.000 \\
\hline 0.89 & 0.094 & 0.060 & 3.065 \\
\hline 1.00 & 0.219 & 0.048 & 9.802 \\
\hline
\end{tabular}

The torsional stiffness distribution and its comparison with the baseline design is shown in Figure 5-2. The optimum section resulted in a torsionally stiffer system as constrained by the optimizer. The resulting extension-twist coupling distribution is depicted in Figure 5-3. It can be seen that the plots verify the negative trend between an increase in torsional stiffness and a decrease in extension-twist coupling. As the coupling values towards the blade tip reduces the torsional stiffness increases. 
In general an increase in the extension-twist coupling would be expected towards the blade tip. However, the target twist rate used as a design objective for this optimization has a constant twist rate. In order to produce such twist rate distribution, the coupling values are increased up to about $80 \%$ of the blade span and reduced to about $20 \%$ of the maximum value at the blade tip.

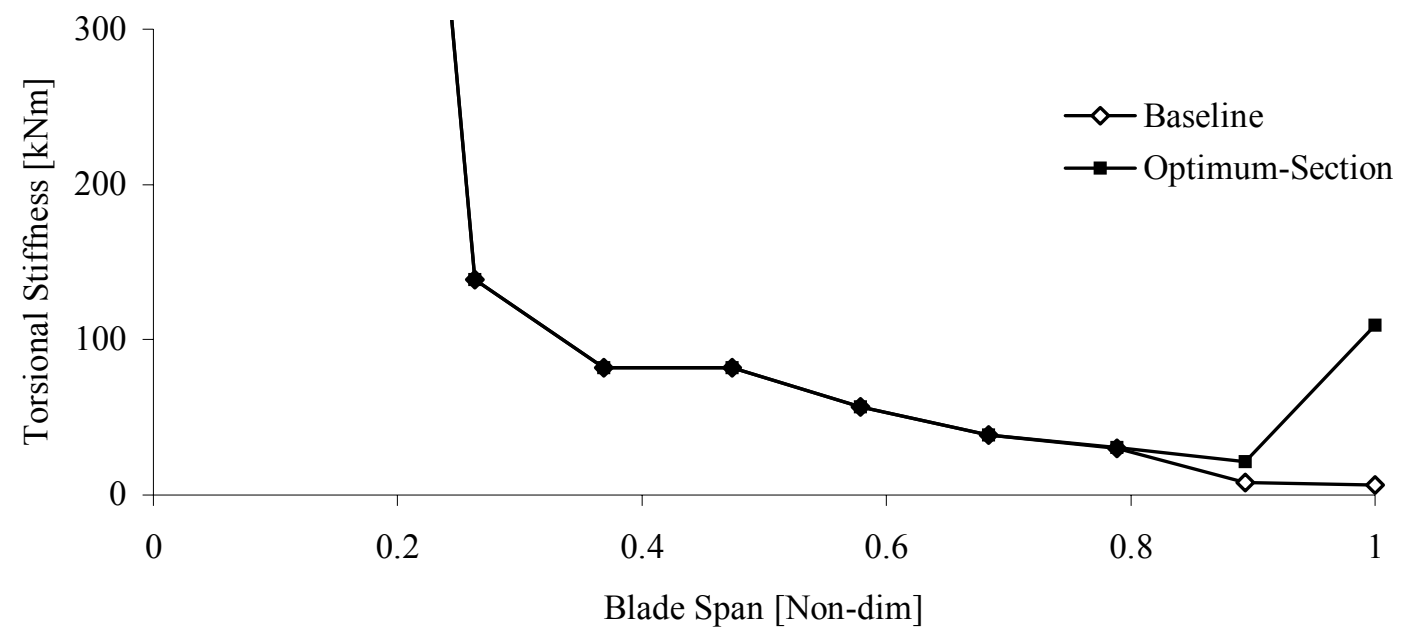

Figure 5-2. Torsional Stiffness Distribution 


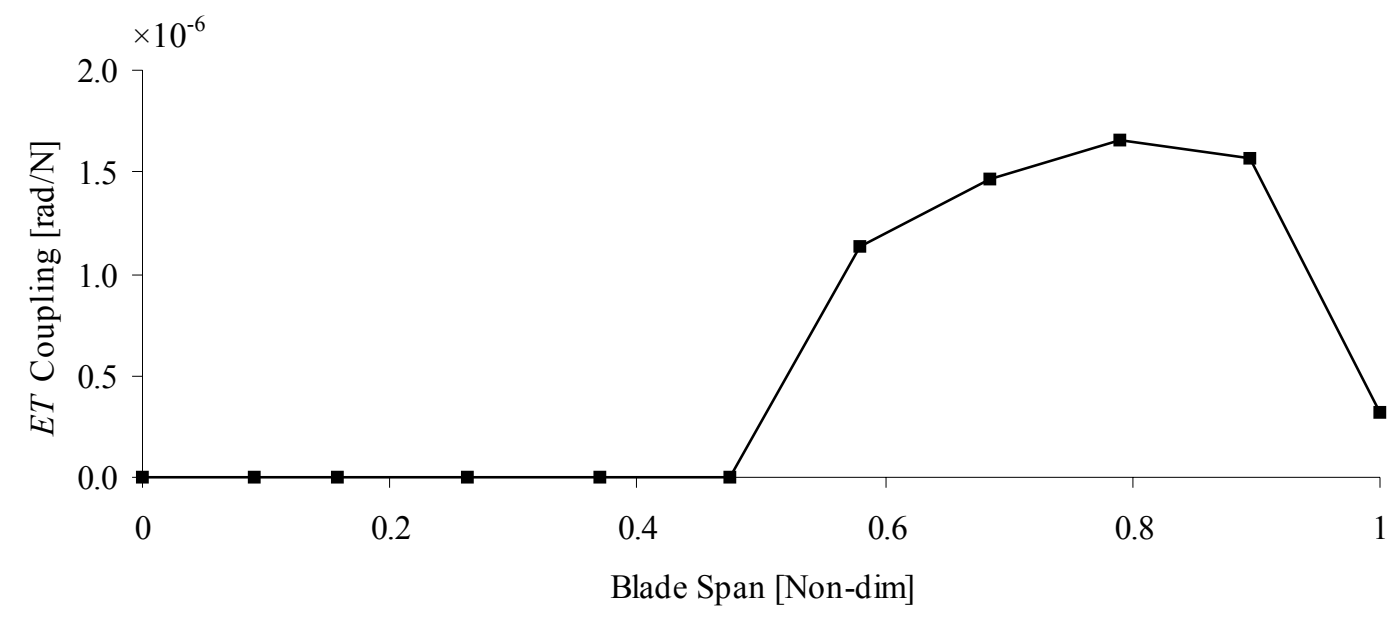

Figure 5-3. Optimum Extension-Twist Coupling Distribution

The final twist distribution for the optimum system and its comparison with the baseline design is shown in Figure 5-4. The non-rotating twist represents the adjusted built-in twist such that when the system runs up to $48 \mathrm{rad} / \mathrm{s}$ the baseline cruise twist distribution is attained while at $59.2 \mathrm{rad} / \mathrm{s}$ the optimum hover twist rate is reached. It can be seen that the optimized system can achieve the target twist distribution within 1 deg difference being higher towards the span location of 0.5. High levels of torsional stiffness requirement around this region limit the levels of extension-twist coupling that can be obtained. Referring back to Figure 5-2 it should be noted that the torsional stiffness of the system is matched with the baseline within $0.5 \%$ between span locations 0.5 to 0.8 hinting to the fact that the limiting coupling values has been reached. 


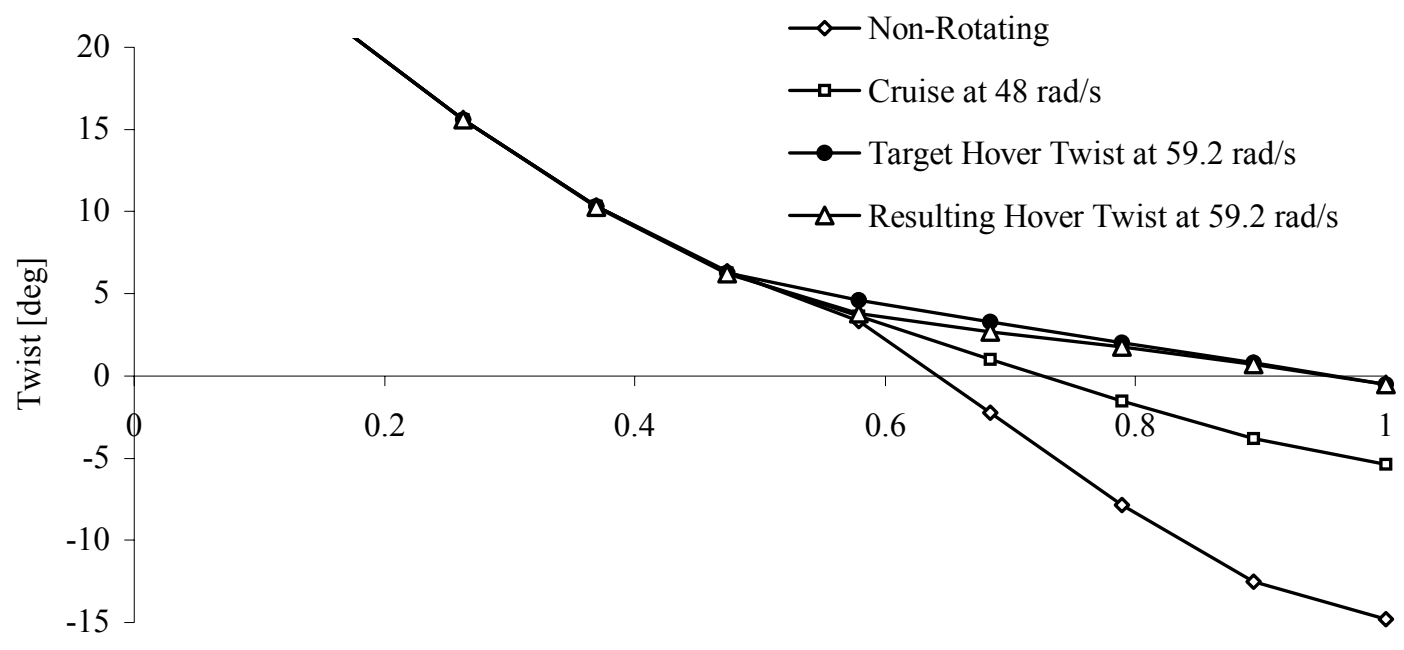

Blade Span [Non-dim]

Figure 5-4. Blade Twist Comparison with the Target Values

The associated power saving with the lower level optimized system is shown in Figure 5-5. The system gives over $6 \%$ improvement compared to the baseline design at $C_{T}=0.01$. This is consistent with the previously discussed saving in hover power requirement for the proposed configuration. 


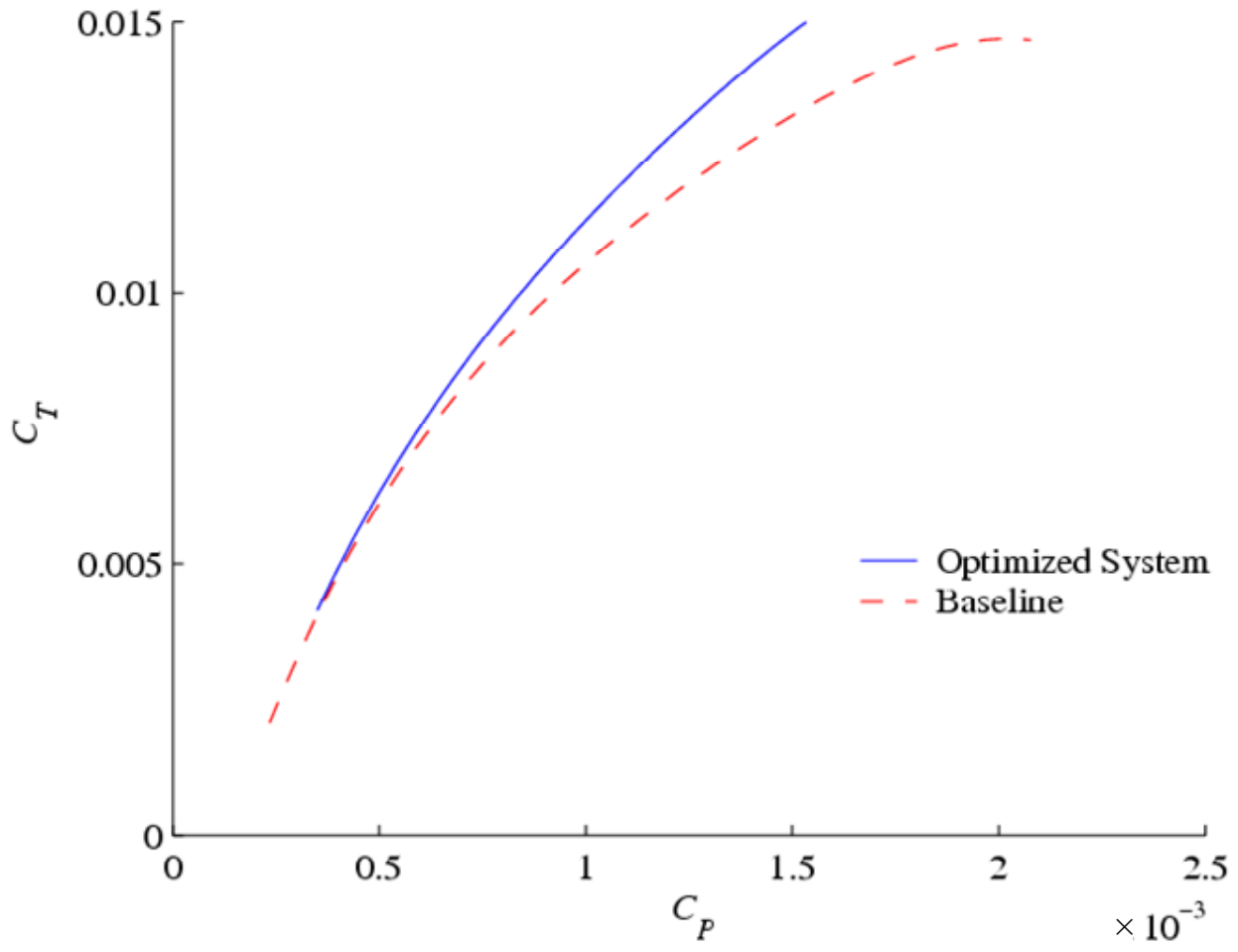

Figure 5-5. Power vs. Thrust Coefficient for Lower-Level Optimized System 


\subsection{Upper Level Optimization}

In the upper level optimization a cross-sectional sizing is performed for a detailed blade sectional model based on the target extension-twist coupling values provided by the lower level optimizer. The optimization is performed at five equally spaced locations along the span of the beam for $R>0.5$.

The change in the cost function with respect to the number generated in the optimizer appears in Figure 5-6. The graph depicts the typical behavior of simulated annealing based algorithms with a quick drop in the value of the cost function within 100 generations but a slow approach to the asymptotic value afterwards. Depending on the computational power at hand and the complexity of the cost function, slow convergence results sometimes 100-200 hours of run time. In this respect it is important to experiment with the optimizer and the system at hand and decide on how far to go in terms of the number generated by the optimizer. For the analysis in this step the optimizer is stopped at 3000 number generations which translated into over 20 hour computation time.

The optimum design parameters for the system are tabulated in Table 5-2 and resulted in an angle ply system with 30 deg angle for the skin. 


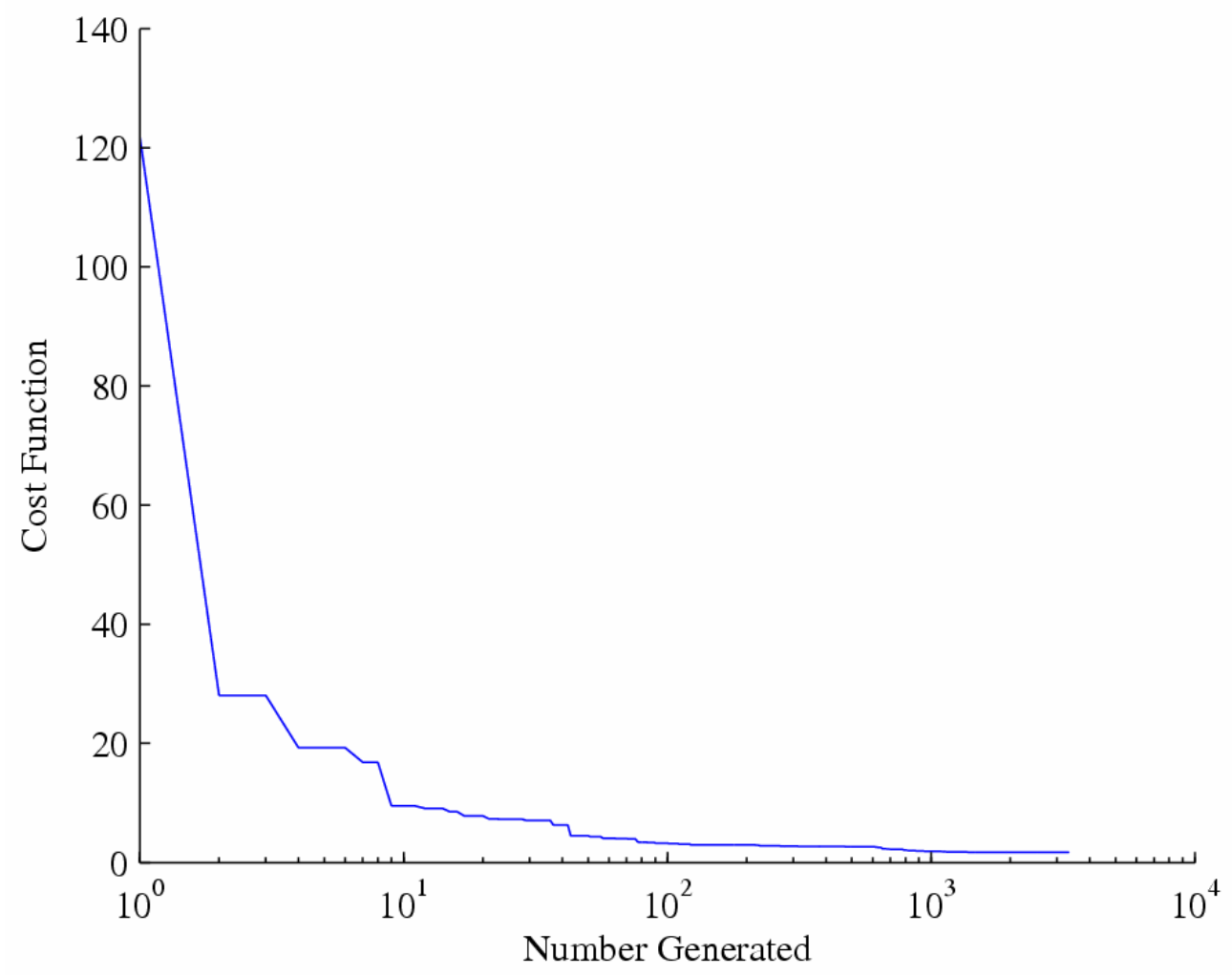

Figure 5-6. Cost Function vs. Number Generated in Simulated Annealing Based Optimizer

Table 5-2 Upper-Level Optimum Design Parameters

\begin{tabular}{|c|c|c|c|c|c|c|c|}
\hline \multirow[b]{2}{*}{ Span } & \multicolumn{3}{|c|}{ Skin } & \multicolumn{3}{|c|}{ Spar } & \multirow[b]{2}{*}{$r_{s} / c$} \\
\hline & $\underline{\theta_{1}[\mathrm{deg}]}$ & $\underline{\theta_{2}[\mathrm{deg}]}$ & $t_{1}[\#$ plies $]$ & $\underline{\theta_{1}[\mathrm{deg}]}$ & $\theta_{2}[\mathrm{deg}]$ & $t_{2}$ [\# plies] & \\
\hline 0.58 & 30 & 30 & 18 & 35 & -80 & 36 & 0.29 \\
\hline 0.68 & 30 & 30 & 8 & 35 & -80 & 72 & 0.25 \\
\hline 0.79 & 30 & 30 & 8 & 35 & -80 & 68 & 0.38 \\
\hline 0.89 & 30 & 30 & 6 & 35 & -80 & 50 & 0.38 \\
\hline 1.00 & 30 & 30 & 4 & 35 & -80 & 80 & 0.39 \\
\hline
\end{tabular}


The ply angles for the spar are found to be 30 and -80 degrees. The composite skin thickness gradually reduced towards the blade tip starting at 18 plies at span location of 0.58 and down to 4 plies at the blade tip, whereas the spar thickness increase towards the blade tip. The spar location gradually increased towards the blade tip. Referring back to Figure 5-3, the tendency of the optimizer to built relatively stiffer section at the blade tip can be explained by the low levels of target coupling values provided by the lower level optimizer at this location.

The comparison of the extension-twist coupling with the target values are shown in Figure 5-7. It can be seen that the target extension-twist coupling distribution is matched within $25 \%$ up to blade span of 0.8 but it is several times off at the blade tip. This is due to the limitation imposed on the composite layup to be constant over the blade span. It shows that with the given stacking sequence it is not possible to tailor that section for the target coupling value within the geometric constraints imposed on the system. A case where the constant composite layup constraint is relaxed and its effects on the coupling values is discussed in the Appendix.

The torsional stiffness together with the flapwise and chordwise bending stiffnesses of the optimized system are given in Figure 5-8 to Figure 5-10. It can be seen that the baseline torsional stiffness is matched closely within about $30 \%$ for span location less than 0.9 and the baseline values are surpassed for span locations greater than 0.9 by over $11 \%$. 


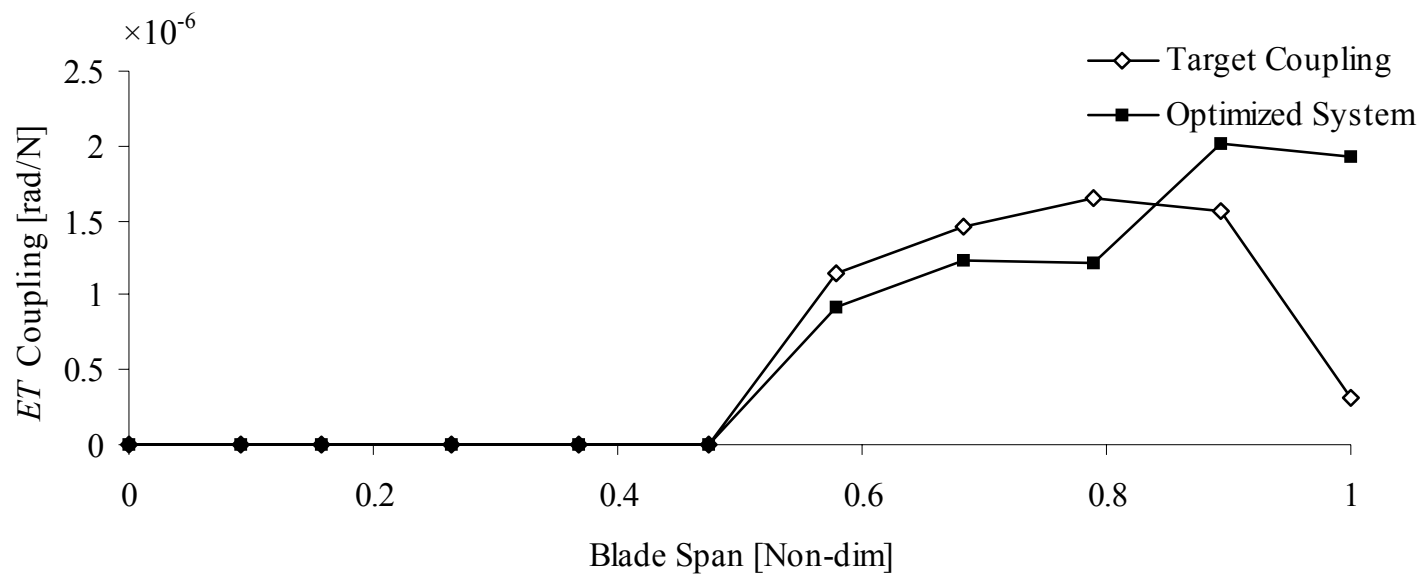

Figure 5-7. Extension-Twist Coupling Distribution

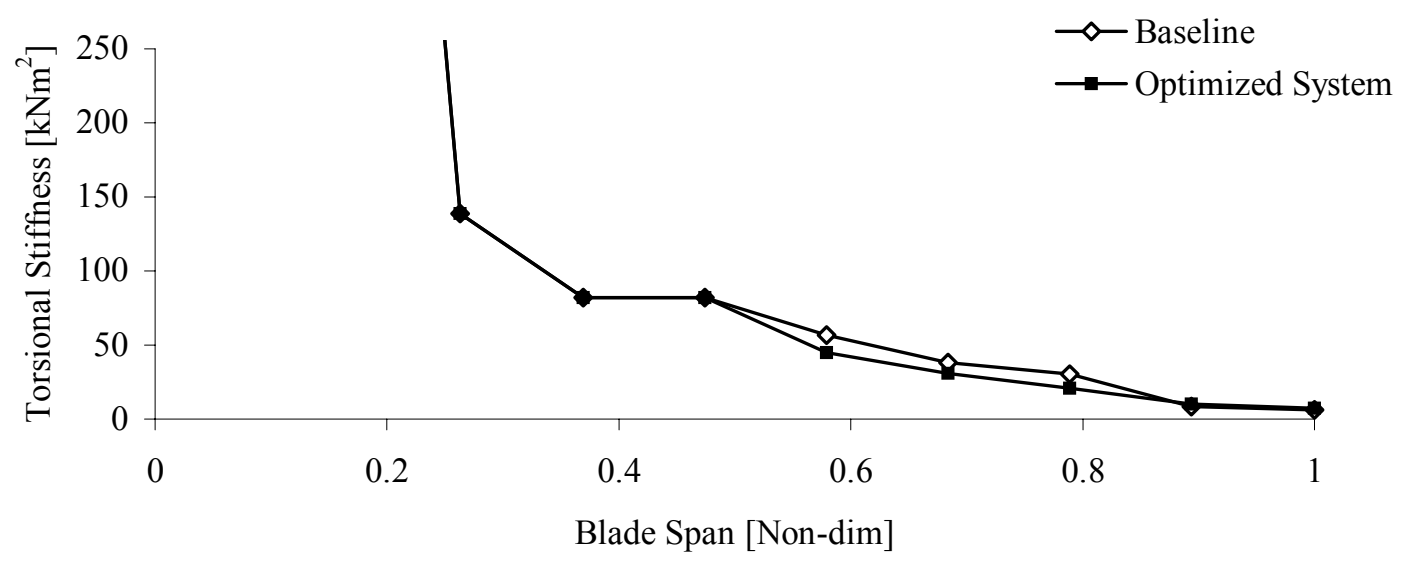

Figure 5-8. Torsional Stiffness Distribution 


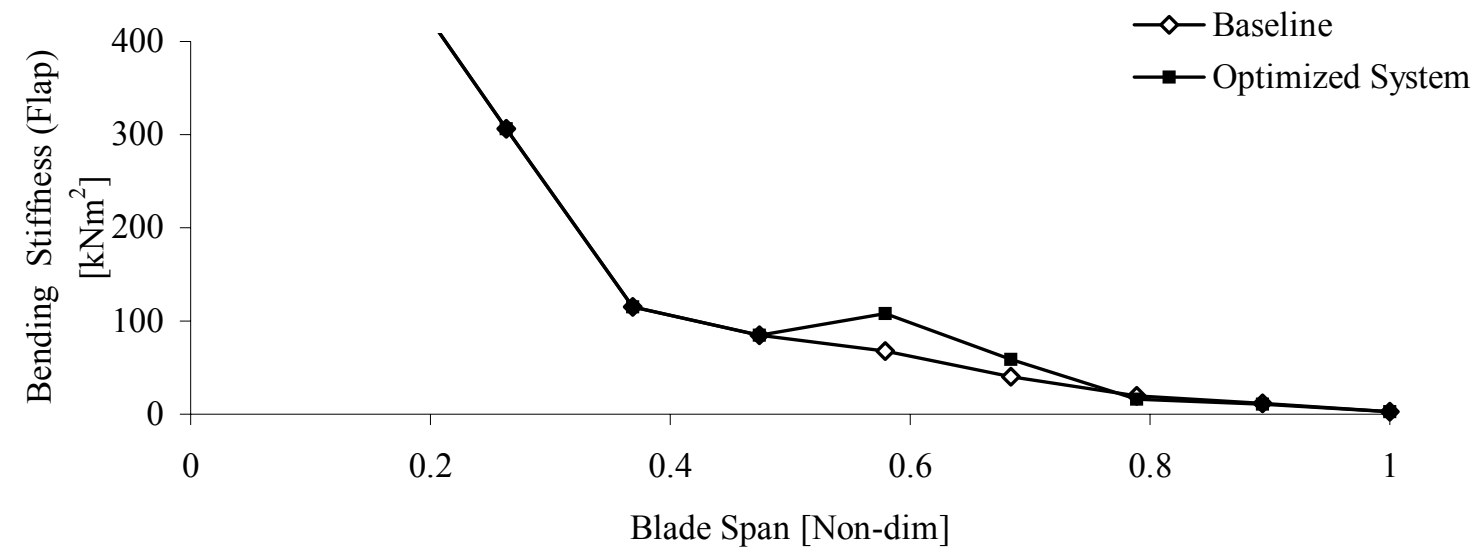

Figure 5-9. Flapwise Bending Stiffness Distribution

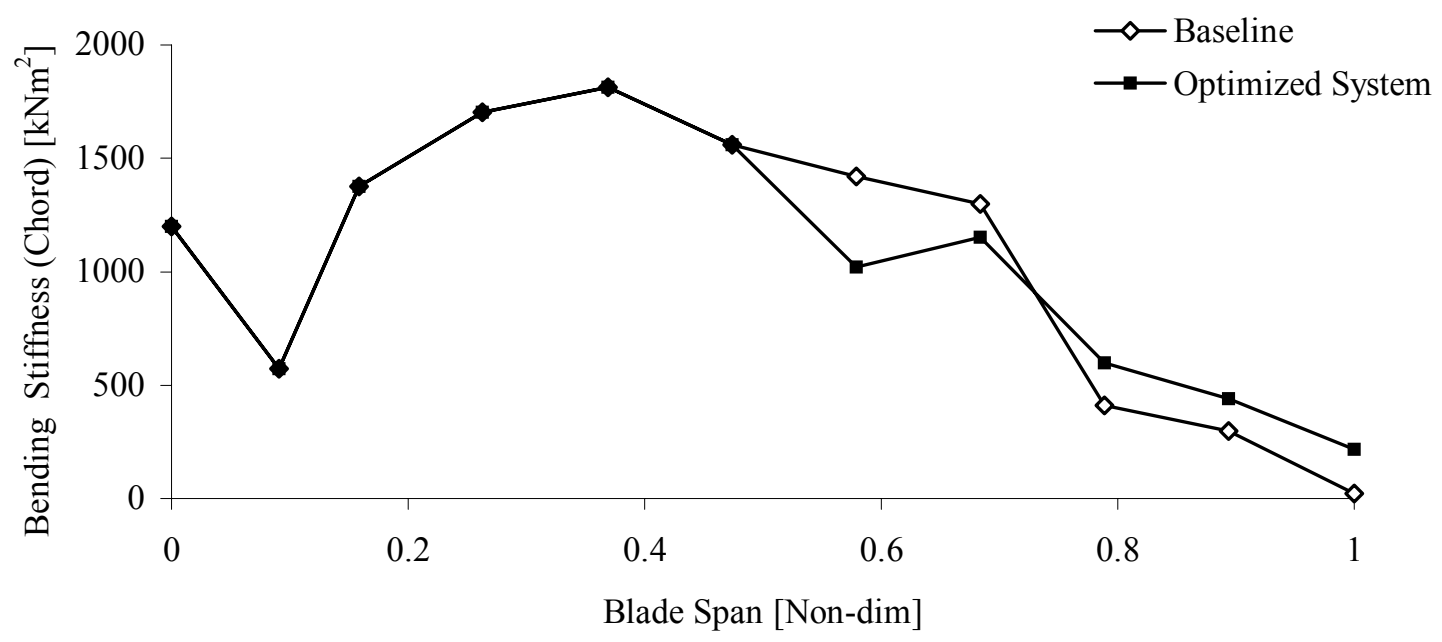

Figure 5-10. Chordwise Bending Stiffness Distribution 
The optimized system resulted in higher flapwise bending stiffnesses for span locations between 0.5 and 0.8 with up to $60 \%$ higher values, but attained lower values towards the blade tip within a maximum difference of $16 \%$. The chordwise bending stiffness however resulted in lower values between span location 0.5 and 0.8 by about $28 \%$ but reached over $50 \%$ higher values towards the blade tip. It should be noted that the stiffness properties of the system matches exactly the baseline design up to the span location 0.5 . In this respect, the overall stiffness characteristics of the rotor blade system are not expected to change drastically as will be discussed later in the stability analysis sections.

The power versus thrust coefficient curve for the system points to about $4.10 \%$ improvement in the hover power requirement with respect to the baseline design with a power coefficient of $C_{P}=8.81 \times 10^{-4}$ at $C_{T}=0.01$, as depicted by Figure 5-11. This is about $70 \%$ of the maximum available power improvement in hover. The system in this case incorporates the sliding mass of $0.997 \mathrm{~kg} / \mathrm{m}$, the optimum value provided by the lower level optimizer. To investigate the effect of the sliding mass on the power requirement, this mass is fixed to the rotor blade throughout the blade span, leaving the tailored cross-section as the only effective term, and the power coefficient is recalculated. This system resulted in $C_{P}=8.89 \times 10^{-4}$ at $C_{T}=0.01$, which translates into $3.21 \%$ improvement in power requirement. With this result it is safe to conclude that about $21 \%$ of the improvement is produced by the sliding mass itself, which points to the additional flexibility the designer can have to tailor the structure without additional weight penalty. 


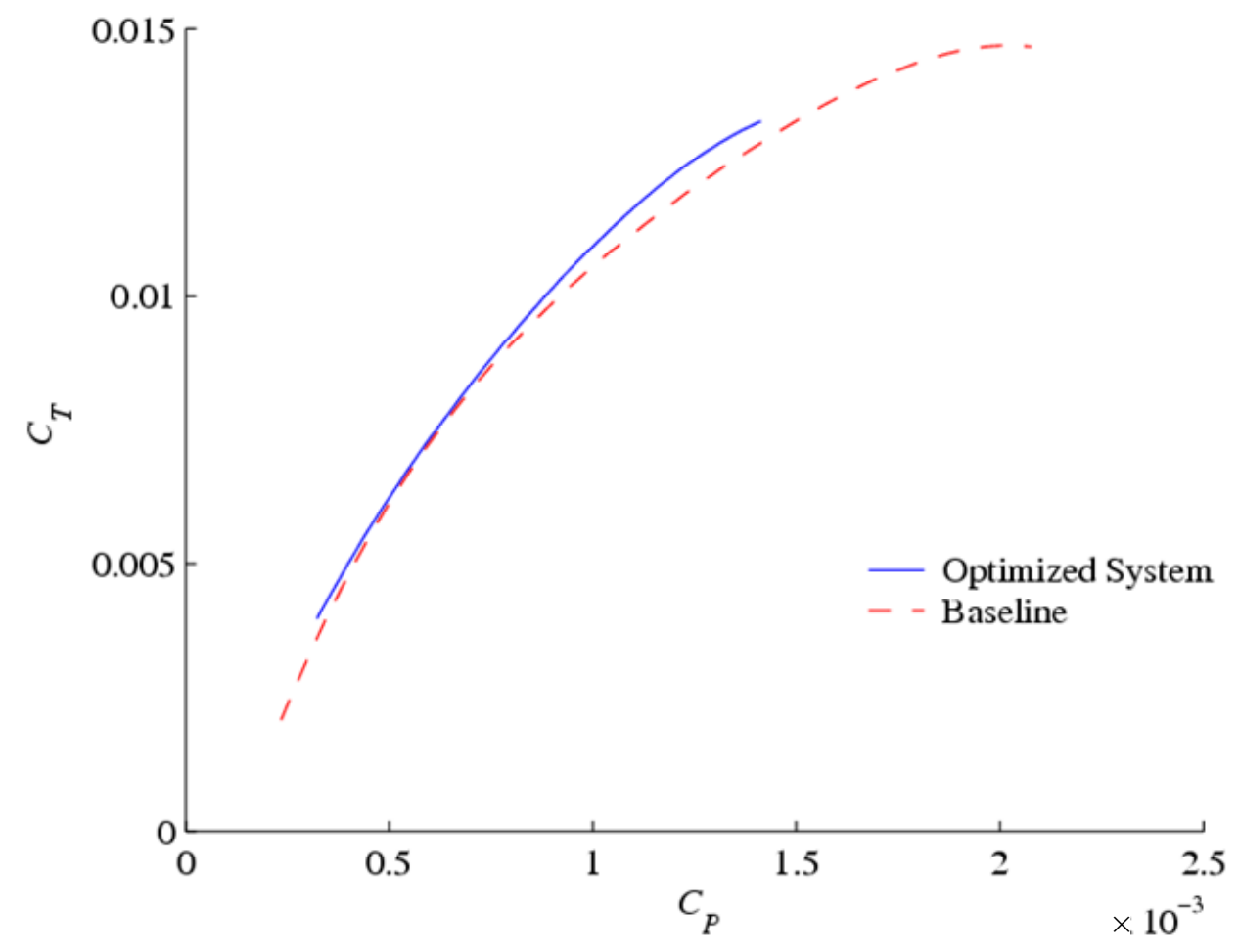

Figure 5-11. Power vs. Thrust Coefficient of the Optimized System

One drawback of the final configuration is the resulting angle ply system resulted for the outer skin of the blade cross-section. Although failure is checked within the optimization process, the failure criteria used does not include failure mechanisms such as ply splitting, one of the main failure mechanisms for angle ply systems. In an attempt to avoid such a failure, the optimized model is strengthened by a $\left[45^{\circ} /-45^{\circ}\right]$ single layer angle-ply composite system on the outside of the blade skin and on the inner surface of the spar. The layup configuration of the new model and an illustration of a cross-sectional model with refined mesh incorporating the additional composite layer are shown in Table 5-3 and Figure 5-12, respectively. 
Table 5-3 Optimized Model Modified Stacking Sequences

\begin{tabular}{|c|c|c|}
\hline Span & Skin & Spar \\
\hline 0.58 & {$\left[30^{\circ}{ }_{18} /-45^{\circ} / 45^{\circ}\right]$} & {$\left[-45^{\circ} / 45^{\circ} / 35^{\circ}{ }_{18} /-80^{\circ}{ }_{18}\right]$} \\
\hline 0.68 & {$\left[30^{\circ}{ }_{8} /-45^{\circ} / 45^{\circ}\right]$} & {$\left[-45^{\circ} / 45^{\circ} / 35^{\circ}{ }_{38} /-80^{\circ}{ }_{38}\right]$} \\
\hline 0.79 & {$\left[30^{\circ}{ }_{8} /-45^{\circ} / 45^{\circ}\right]$} & {$\left[-45^{\circ} / 45^{\circ} / 35^{\circ}{ }_{34} /-80^{\circ}{ }_{34}\right]$} \\
\hline 0.89 & {$\left[30^{\circ}{ }_{6} /-45^{\circ} / 45^{\circ}\right]$} & {$\left[-45^{\circ} / 45^{\circ} / 35^{\circ}{ }_{25} /-80^{\circ}{ }_{25}\right]$} \\
\hline 1.00 & {$\left[30^{\circ}{ }_{4} /-45^{\circ} / 45^{\circ}\right]$} & {$\left[-45^{\circ} / 45^{\circ} / 35^{\circ}{ }_{40} /-80^{\circ}{ }_{40}\right]$} \\
\hline
\end{tabular}

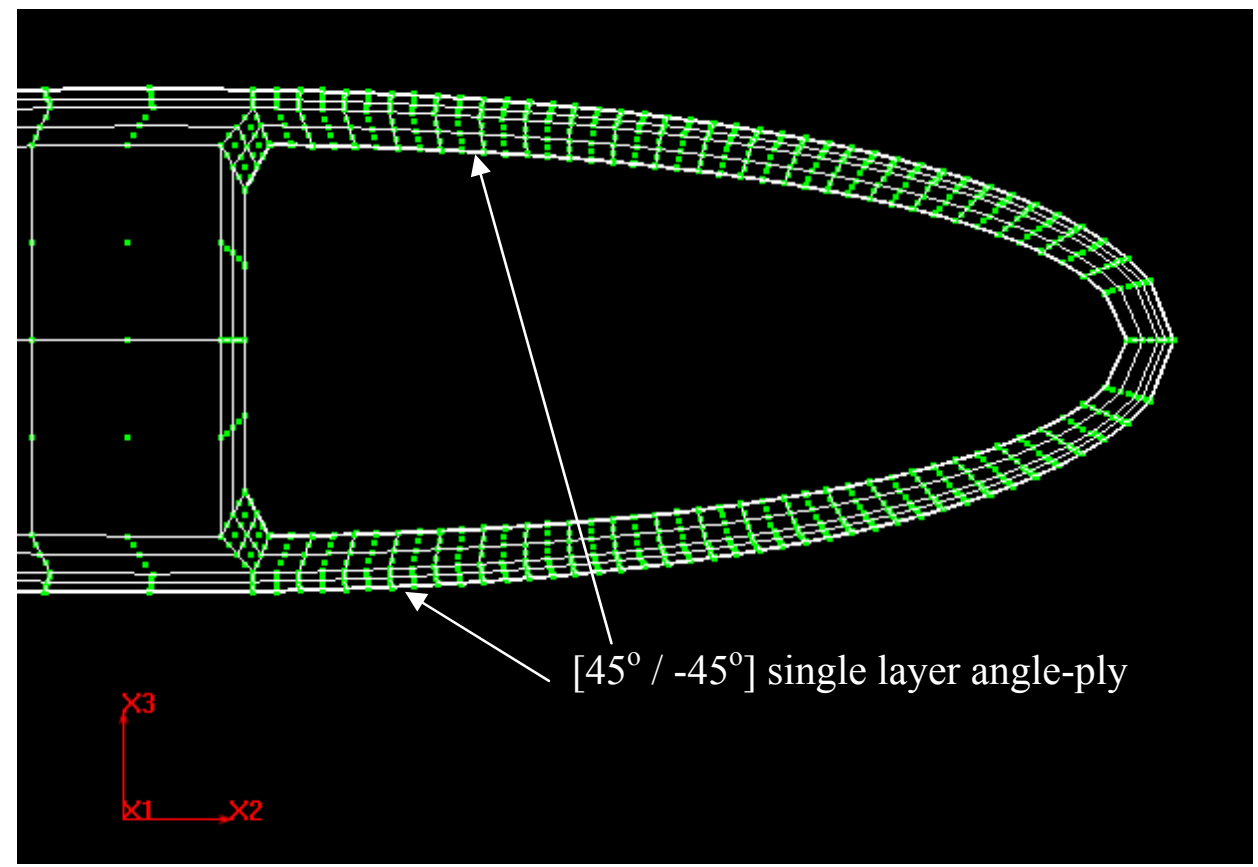

Figure 5-12. Single Layer of $\left[45^{\circ} /-45^{\circ}\right]$ Angle-ply Application on the Model 
The effect of the angle-ply layer on the extension-twist coupling of the optimized system is presented in Figure 5-13. It can be seen that the coupling values are reduced with respect to the angle-ply system from $10 \%$ to over $45 \%$, depending on the span location. At span locations where the cross-ply system constitutes a higher proportion of the total material used, the drop is more drastic. However, at some span locations such as 0.9 and 1.0 the model benefits from the cross-ply layers as the coupling values are brought closer to the target values.

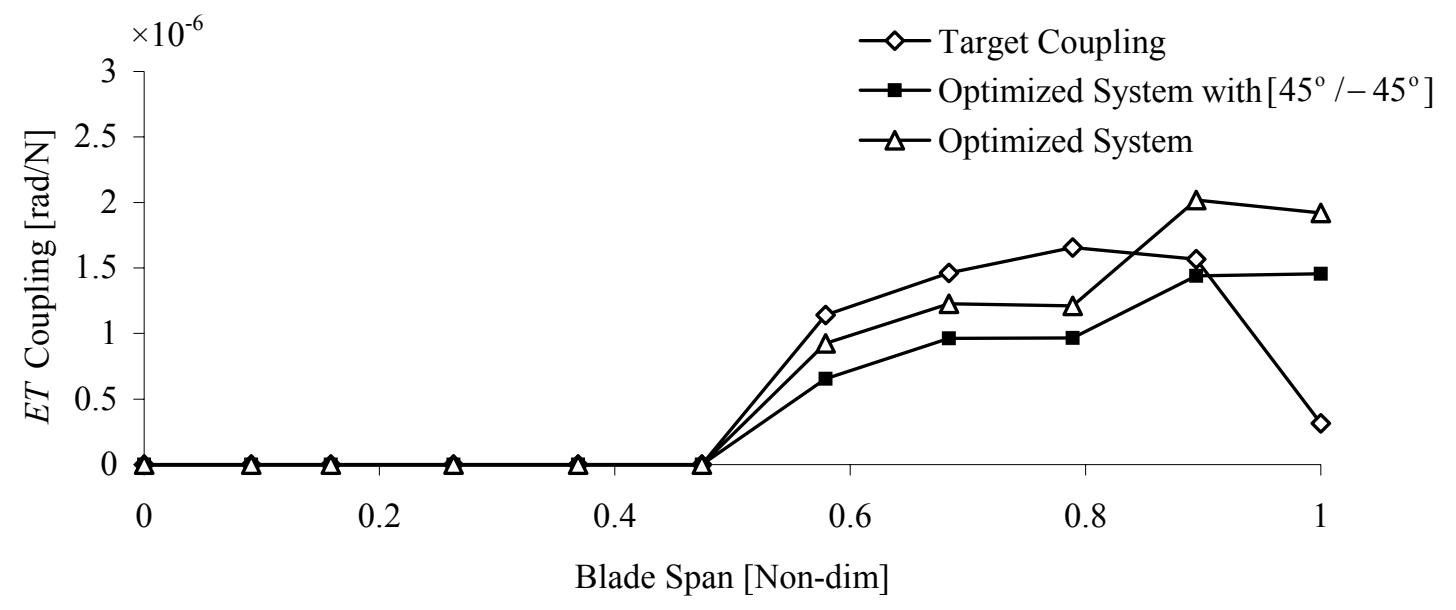

Figure 5-13. Extension-Twist Coupling Distribution for the Modified System 
The torsional stiffness together with the flapwise and chordwise bending stiffnesses of the model with the new layup configuration is shown in Figure 5-14 to Figure 5-16. The new system resulted in over $20 \%$ torsionally stiffer cross-section for span locations greater than 0.9 , and the maximum difference compared to the baseline dropped to around $25 \%$.

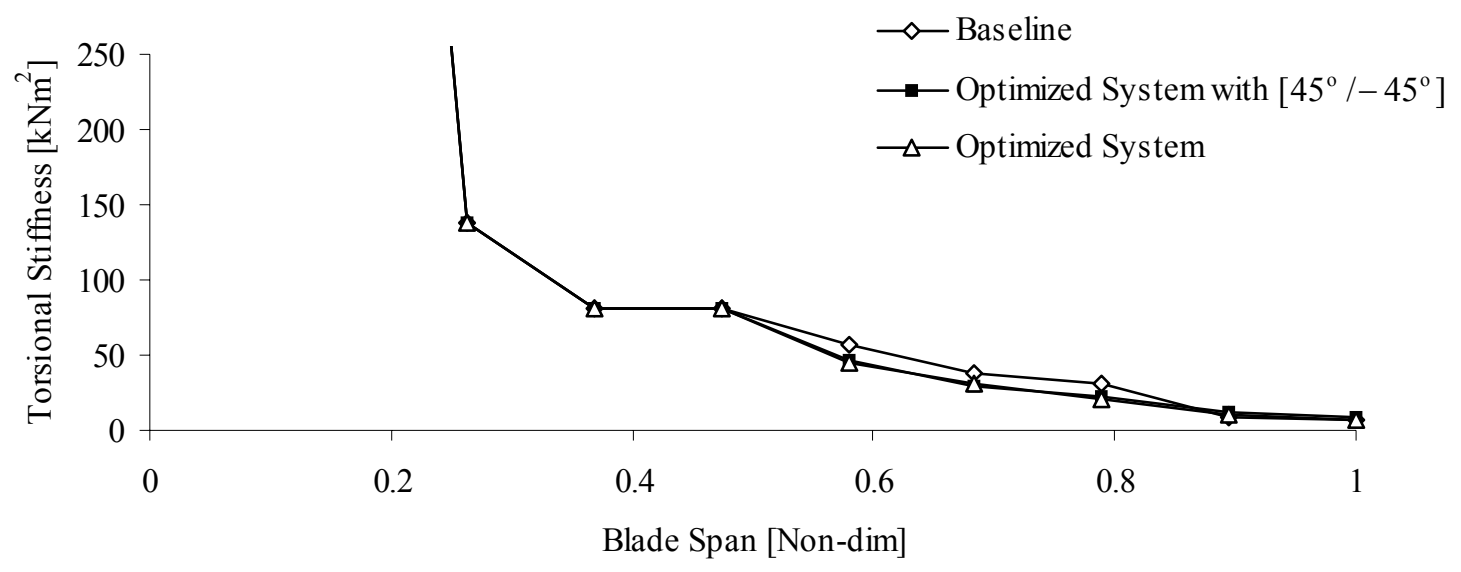

Figure 5-14. Torsional Stiffness Distribution of the Modified System 


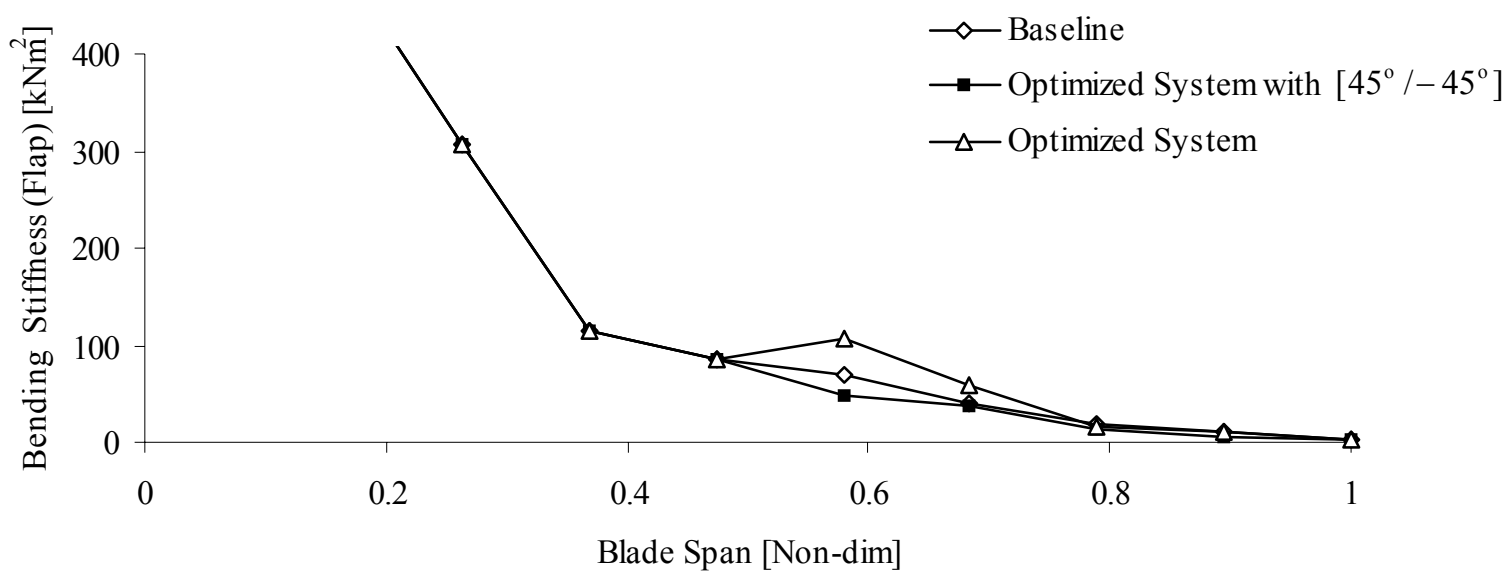

Figure 5-15. Flapwise Bending Stiffness Distribution of the Modified System

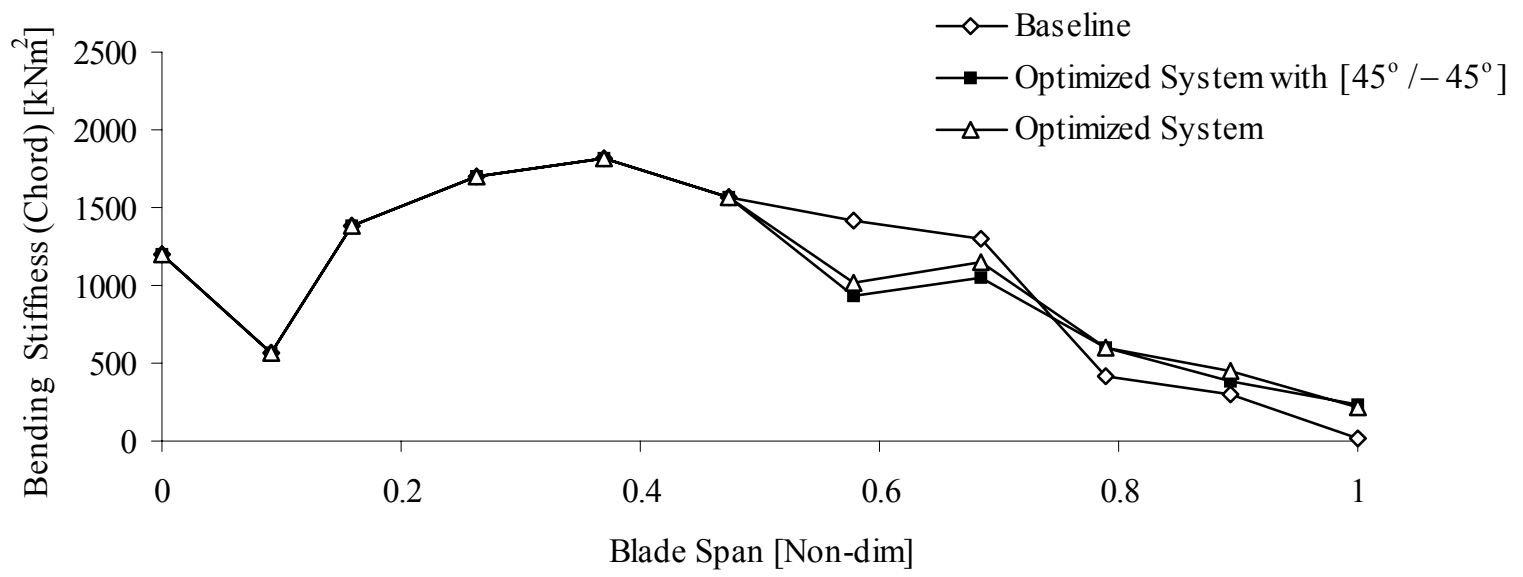

Figure 5-16. Chordwise Bending Stiffness Distribution of the Modified System 
Both flapwise and bending stiffnesses showed a drop compared to the optimized system, being more pronounced in the flapwise bending stiffness. An increase would be expected as the additional cross-ply system would make the structure stiffer. However, it should be noted that the blade structure is actually an initially twisted beam. In this respect the stiffness matrix inherits couplings which are not only a function of the material anisotropy but also a function of beam geometry. Hence this drop might be attributed to the interactions between the stiffness and the geometry couplings. The effect of the angle-ply system on the untwisted cross-section illustrates the expected increase in the stiffness values as depicted by Figure 5-17 and Figure 5-18.

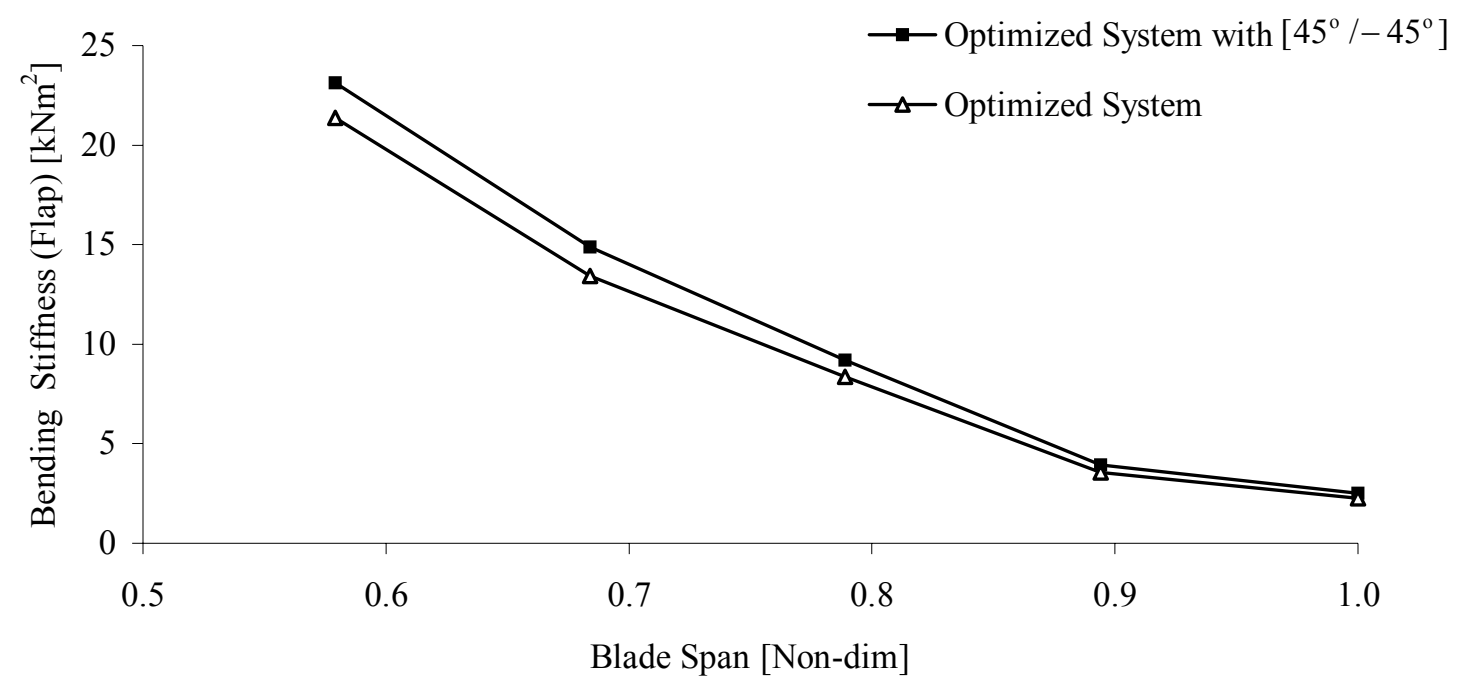

Figure 5-17. Untwisted Model Flapwise Bending Stiffness 


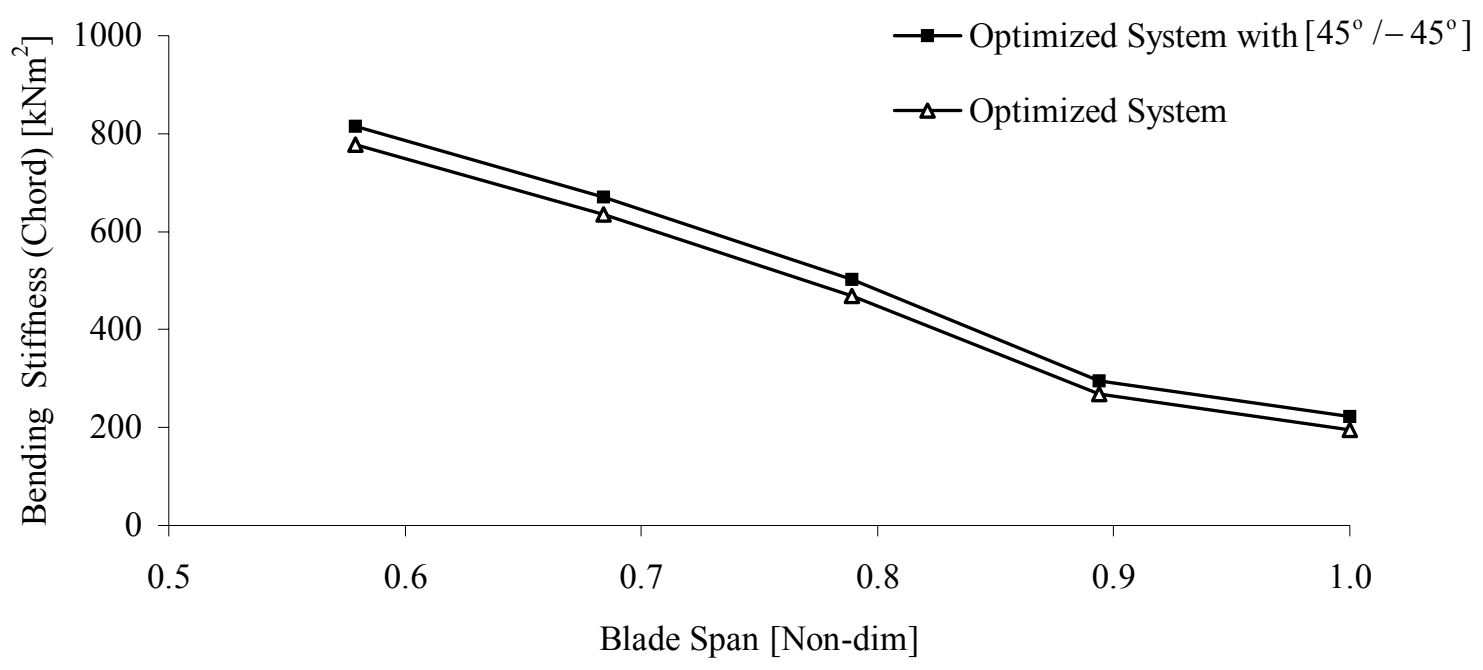

Figure 5-18. Untwisted Model Chordwise Bending Stiffness

The effect of the $\left[45^{\circ} /-45^{\circ}\right]$ angle-ply system on the final twist at hover is shown in Figure 5-19. It can be seen that there is a slightly more than 1 degree penalty in the final twist achieved at the blade tip compared to the original optimized system.

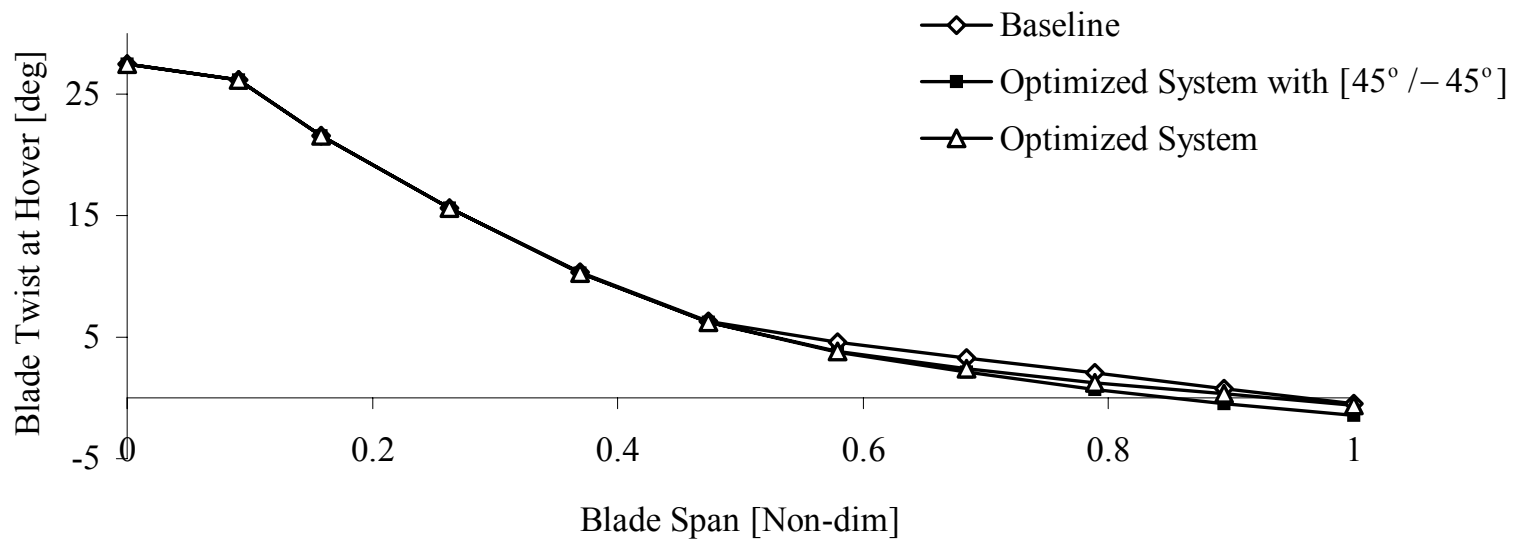

Figure 5-19. Effect of the $\left[45^{\circ} /-45^{\circ}\right]$ Angle-ply Layer on the Final Twist Distributions 
With this twist rate, the power versus thrust coefficient curve for the new system points to about $3.76 \%$ improvement in the hover power requirement with respect to the baseline design for a power coefficient of $C_{P}=8.84 \times 10^{-4}$ at $C_{T}=0.01$, as shown by Figure $5-20$. It is about $8 \%$ less than the original optimized system, a penalty associated with the use of a practical system.

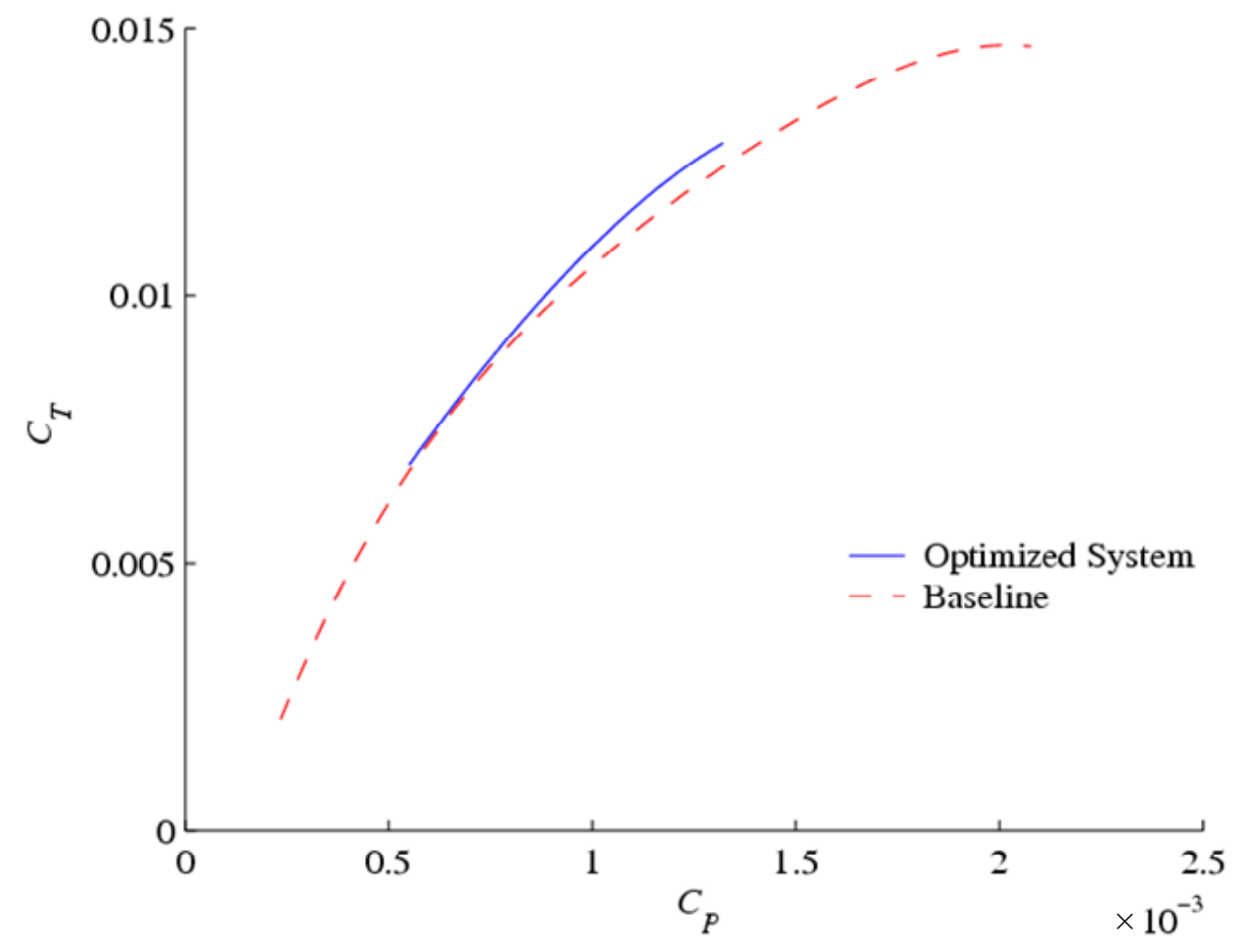

Figure 5-20. Power vs. Thrust Coefficient for the System with the $\left[45^{\circ} /-45^{\circ}\right]$ Angle-ply Layer 
One important feature of the final design is its hygrothermal stability. To test the response of the final optimized system to temperature change a representative blade crosssection is analyzed in ABAQUS. For the analysis, a simplified uniform cross-section blade model with geometric properties and stacking sequence similar to that of blade span 0.79 is generated as shown in Figure 5-21. The 0.79 span location is chosen as a representative section since any other section will either be torsionally stiffer or will have higher proportions of $\left[45^{\circ} /-45^{\circ}\right]$ stacking sequence, making the section more stable to temperature change. The geometric and material properties of the model are tabulated in Table 5-4.

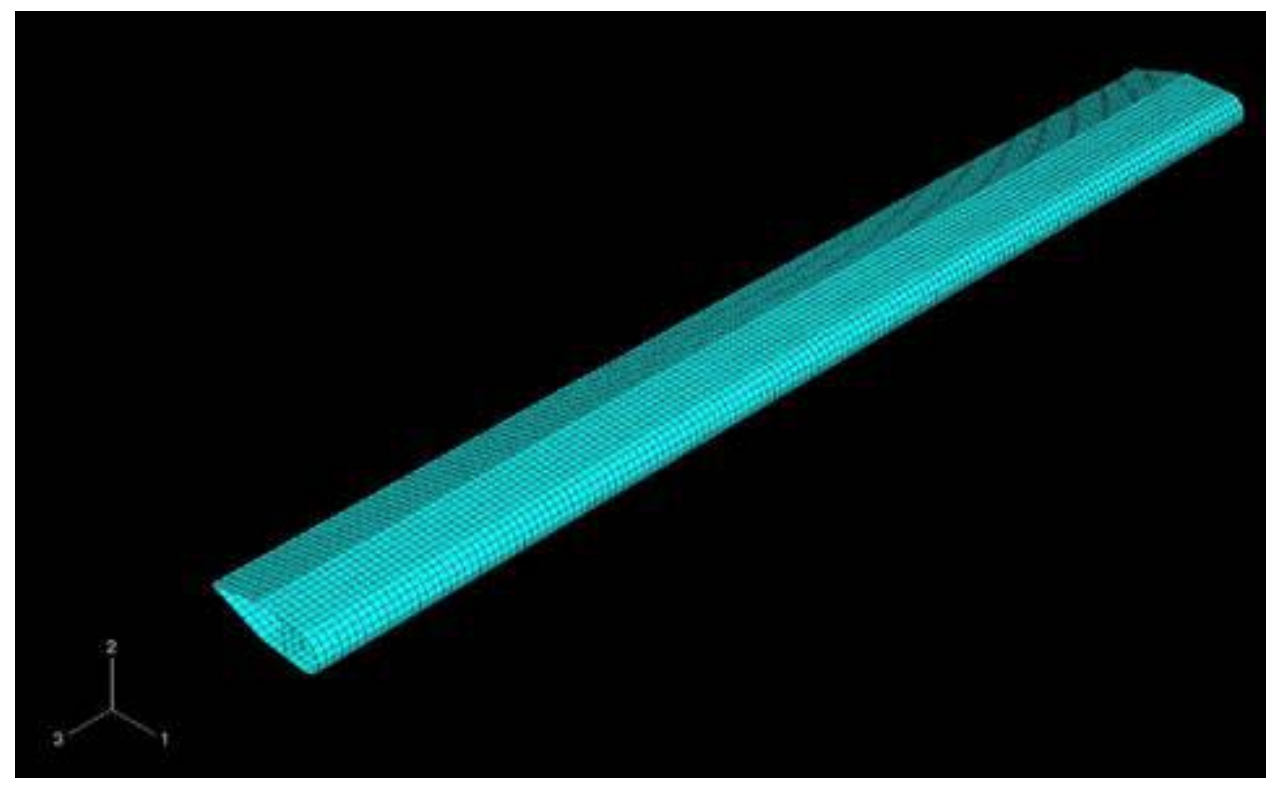

Figure 5-21. Representative ABAQUS Model 
Table 5-4 ABAQUS Model Geometric and Material Properties

\begin{tabular}{lll} 
Geometry & & \\
\hline Chord & 0.355 & $\mathrm{~m}$ \\
Thickness & 0.1 & $\mathrm{~m}$ \\
Span & 3 & $\mathrm{~m}$ \\
Material & & \\
\hline$E_{11}$ & 138 & $\mathrm{GPa}$ \\
$E_{22}$ & 8.96 & $\mathrm{GPa}$ \\
$G_{12}=G_{13}$ & 7.1 & $\mathrm{GPa}$ \\
$G_{23}$ & 3.4 & $\mathrm{GPa}$ \\
$v_{12}$ & 0.3 & \\
$\alpha_{11}$ & $4.20 \times 10^{-6}$ & ${ }^{\circ} \mathrm{C}^{-1}$ \\
$\alpha_{22}$ & $3.70 \times 10^{-5}$ & ${ }^{\circ} \mathrm{C}^{-1}$ \\
\multicolumn{4}{l}{ Stacking Sequence } & \\
\hline Skin & {$\left[30^{\circ}{ }_{12} /-45^{\circ} / 45^{\circ}\right]$} \\
Spar & {$\left[45^{\circ} /-45^{\circ} / 35^{\circ}{ }_{29} /-80^{\circ}{ }_{29}\right]$}
\end{tabular}

A uniform temperature change of $100{ }^{\circ} \mathrm{C}$ has been assumed. Through a standard analysis it has been observed that the response of the structure to the temperature change is minimal with $0.22 \mathrm{deg} / \mathrm{m}$ twist rate for $100{ }^{\circ} \mathrm{C}$ temperature change as shown in Figure 5-22. Hence it is safe to state that the structure is hygrothermally stable within the tolerances assumed in the present work. 


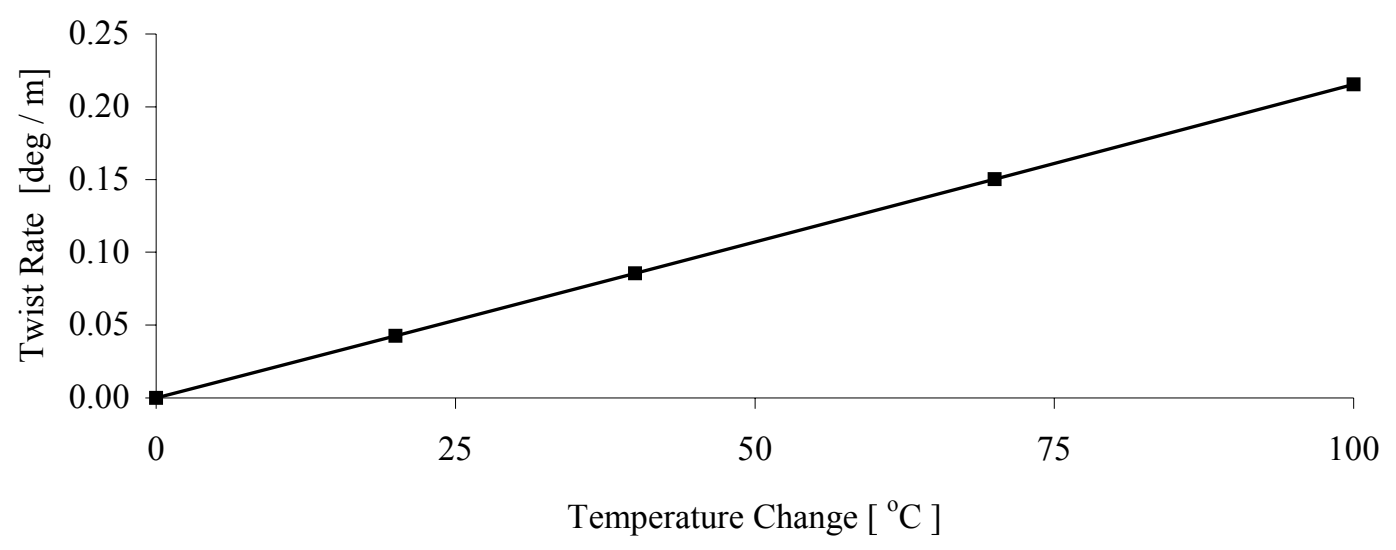

Figure 5-22. Twist Rate vs. Temperature Change of the Representative Model

\subsection{Aeroelastic Stability}

The aeroelastic stability of the optimized model with the $\left[45^{\circ} /-45^{\circ}\right]$ angle-ply system in cruise mode is investigated using DYMORE. The analysis is based on examining the time simulations of the system due to different initial conditions using Prony series approximations.

The analysis of the system is performed by exciting the flap, lag and torsion/pylon modes of the wing using initial displacement conditions. The initial conditions are realized by applying concentrated wing tip loads quasi-statically and restarting the analysis in the dynamic mode upon releasing the tip load. The system is allowed to vibrate for about 5 seconds and system displacement and force measurements are recorded at various wing and blade locations. Wing root moment data are used for analyzing the system parameters. The form of the Prony series fit is shown in Eqn. 3. Here $N$ denotes the number of modes 
considered for the analysis and is determined based on minimizing the squared error between the fit and the data. The damping and damped frequency are represented by $\zeta, \omega$, respectively. A typical fit to the data is depicted in Figure 5-23. The percent critical damping values are then determined by dividing the damping value with the corresponding in-vacuum natural frequency.

$$
f(t)=\operatorname{Re}\left[\sum_{k=1}^{N} e^{\left(\zeta_{k}+i \omega_{k}\right) t}\right]
$$

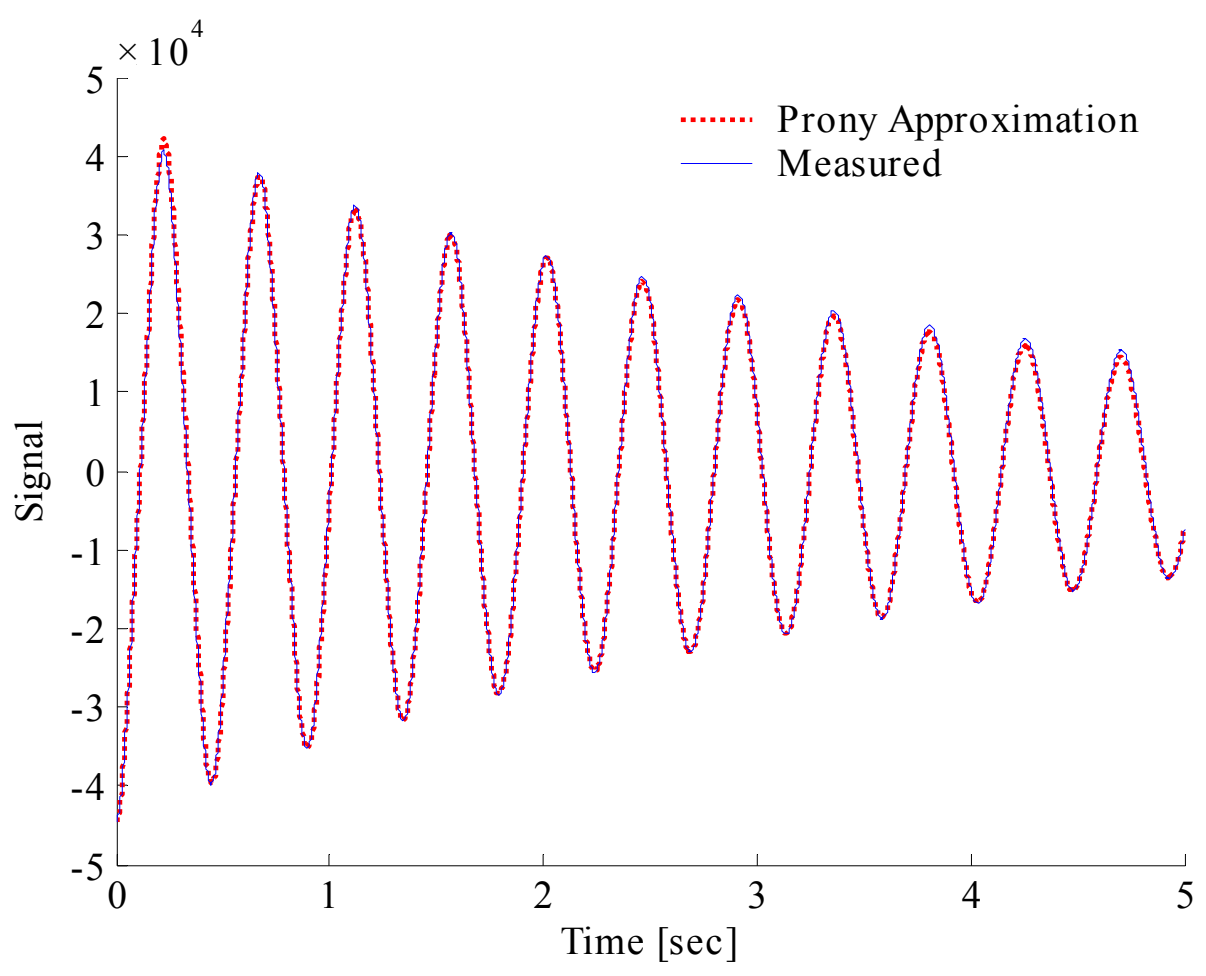

Figure 5-23. Typical Prony Series Fitting 
The comparison of the isolated rotor frequencies of the system with the $\left[45^{\circ} /-45^{\circ}\right]$ angle-ply layup versus the baseline design is shown in Figure 5-24. As discussed previously the systems total mass properties are kept as the baseline design; however there is a difference in the final stiffness properties and the optimized system is more compliant at some span locations. These are reflected by the frequencies: The first three non-rotating rotor frequencies are within $6 \%$ those of the baseline. For the higher frequencies there is a reduction in the optimized system up to $25 \%$, which can be attributed to the reduced stiffnesses at some span locations. All frequencies increase with rotor speed. The same reduction is observed in the optimized system frequencies at the vehicle operating conditions.

The system wing flapwise bending mode (WFBM), wing chordwise bending mode (WCBM) and wing torsion/pylon mode (WTPM) frequencies in vacuum are determined using the eigenmode extraction capability of DYMORE. As tabulated in Table IV, the optimized system wing mode natural frequencies are within $4 \%$ of the baseline. This is expected since the total mass of the blade together with the majority of the blade stiffness is very close to the baseline system.

The system is trimmed for $C_{T}=0.00445$ at $10,000 \mathrm{ft}$. with a rotor speed of $48 \mathrm{rad} / \mathrm{s}$ and the system properties are determined under powered flight mode. The damping characteristics of the baseline system are given in Figure 5-25. It can be seen that the system is stable for the selected cruise speed variation. An increase in the flapping mode damping is observed as the cruise speed increases. The chordwise and torsion/pylon damping decrease slightly with air speed.

Figure 5-26 depicts the damping values for the system with optimum stiffness distribution. It is found that the system is stable under the chosen operating condition. The 
damping values show similar trend compared to the baseline model. The relative difference in the damping characteristics among the two cases at an airspeed of 310 Knots is shown in Figure 5-27. It is seen that the damping ratios show a slight reduction compared to the baseline model. The highest reduction is noted in the flapwise-bending mode damping ratio with $8 \%$. The reduction in the chordwise bending mode damping ratio on the other hand is the smallest with $1.6 \%$.

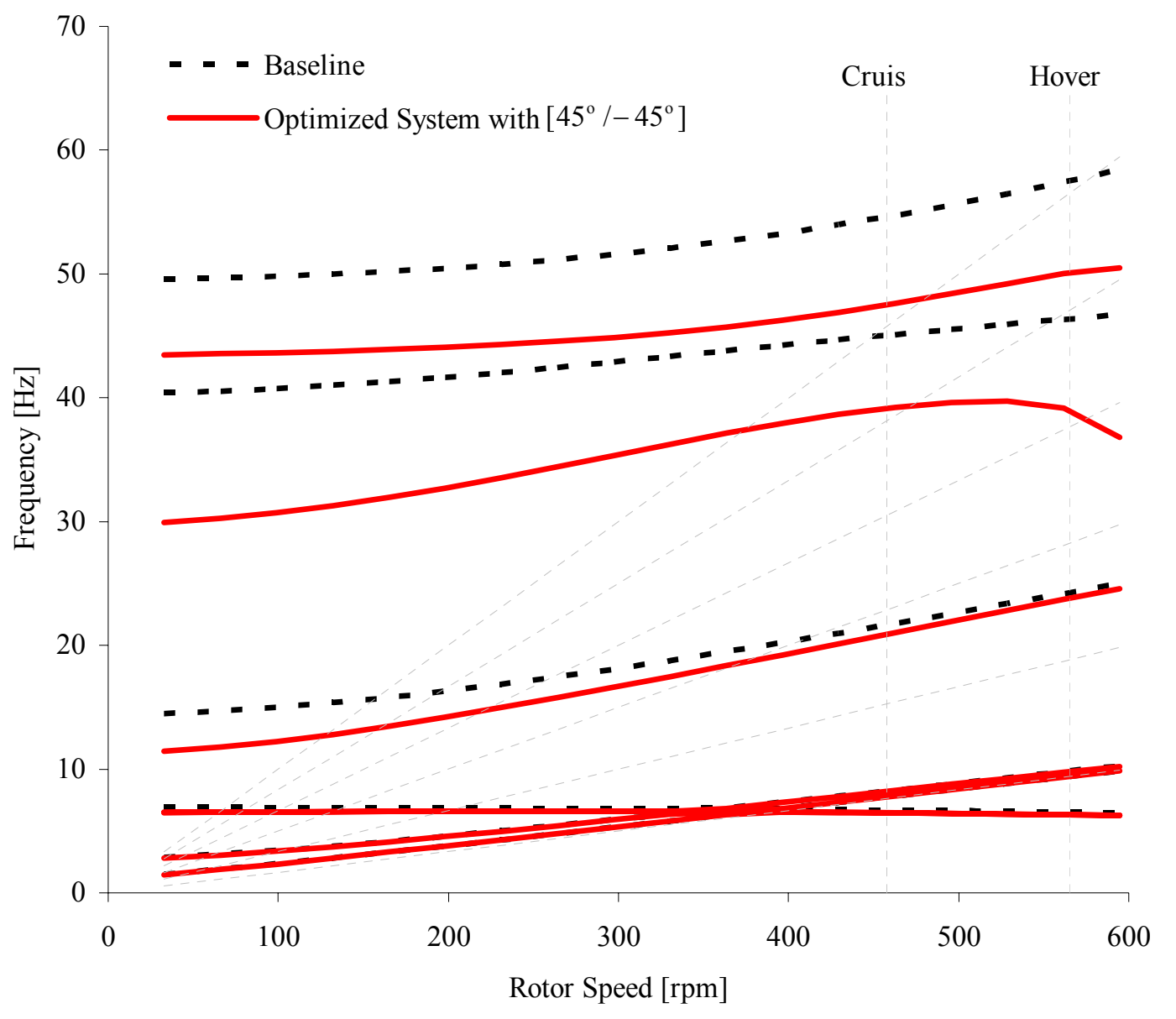

Figure 5-24. Isolated Rotor Frequency vs. Rotor Speed 
Table 5-5 Wing Mode Natural Frequency in Vacuum [Hz]

\begin{tabular}{cccc} 
& WFBM & WCBM & WTPM \\
\hline Baseline & 2.069 & 4.277 & 5.574 \\
Optimized System with $\left[45^{\circ} /-45^{\circ}\right]$ & 2.055 & 4.263 & 5.365
\end{tabular}

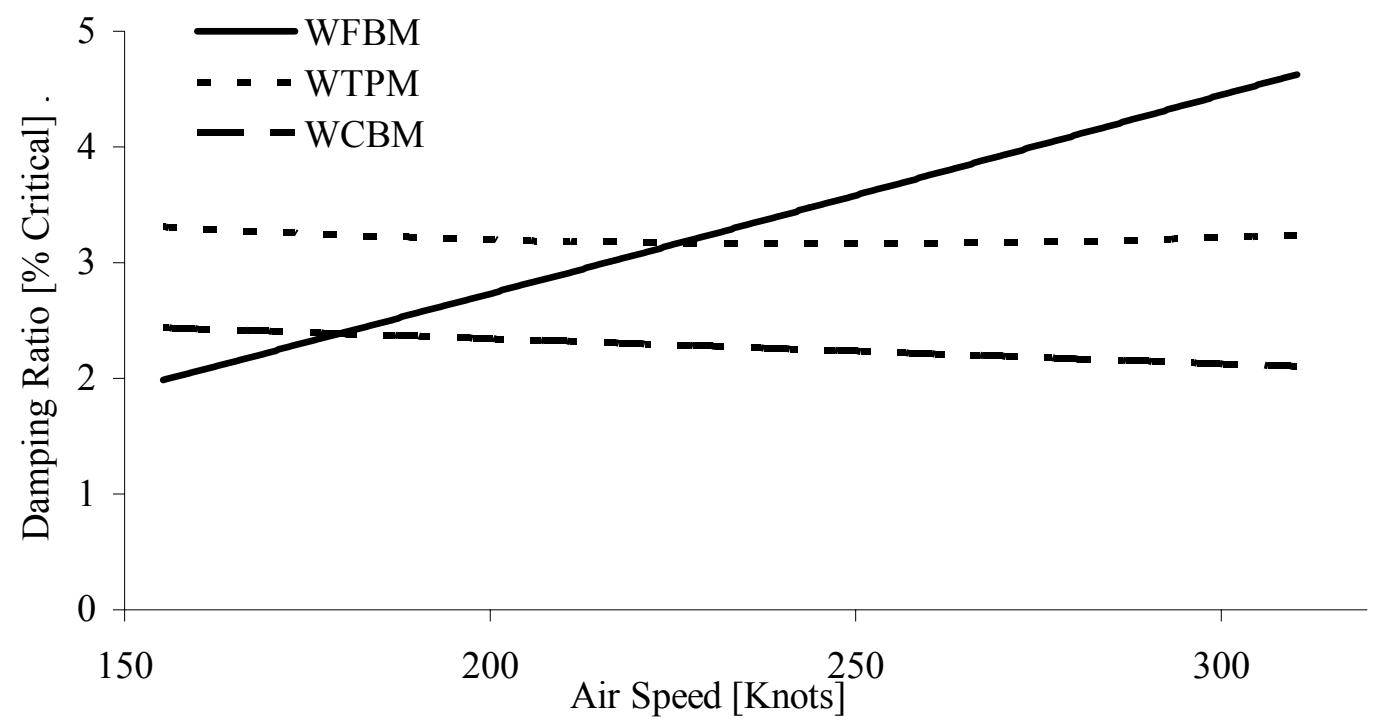

Figure 5-25. Baseline Damping Ratios 


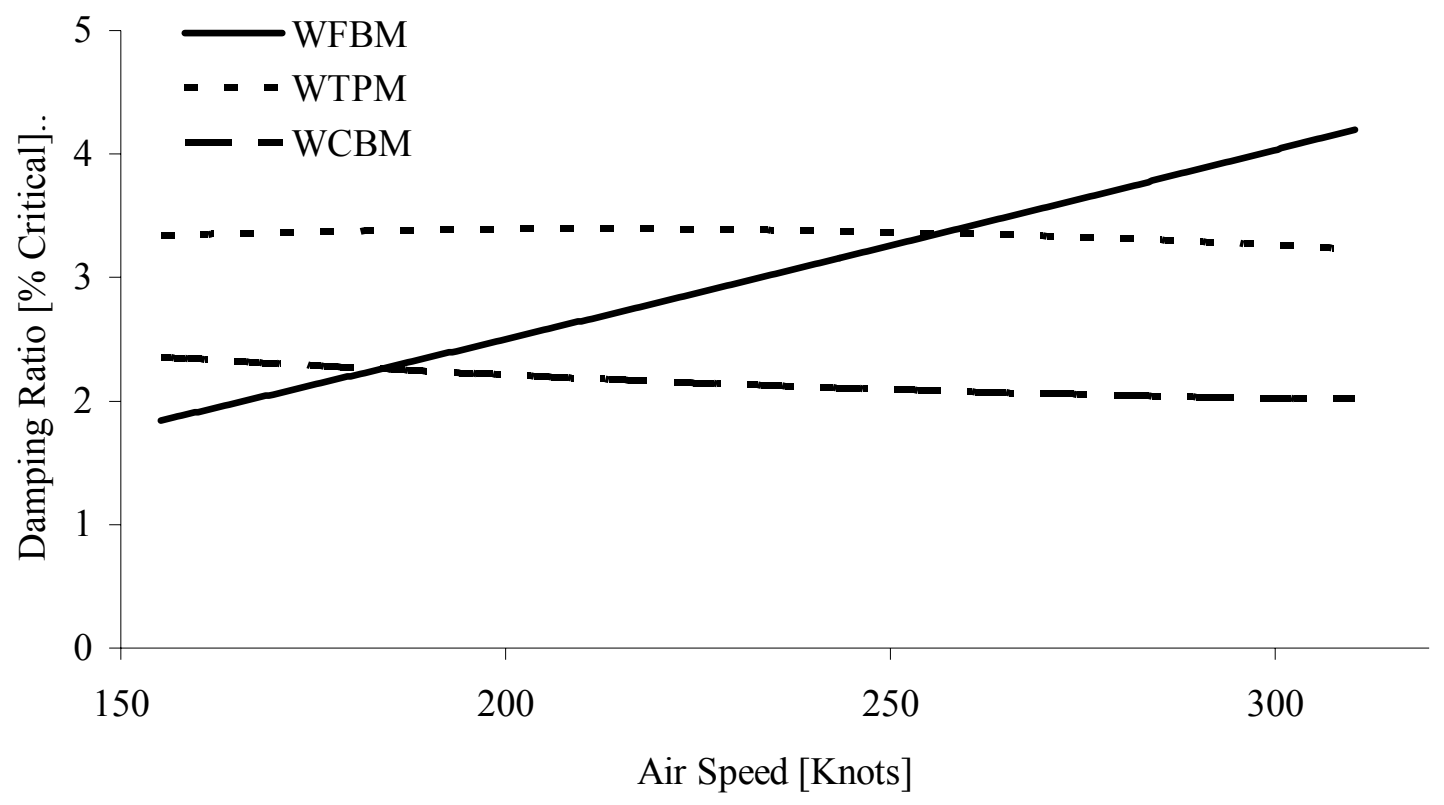

Figure 5-26. Optimized System Damping Ratios

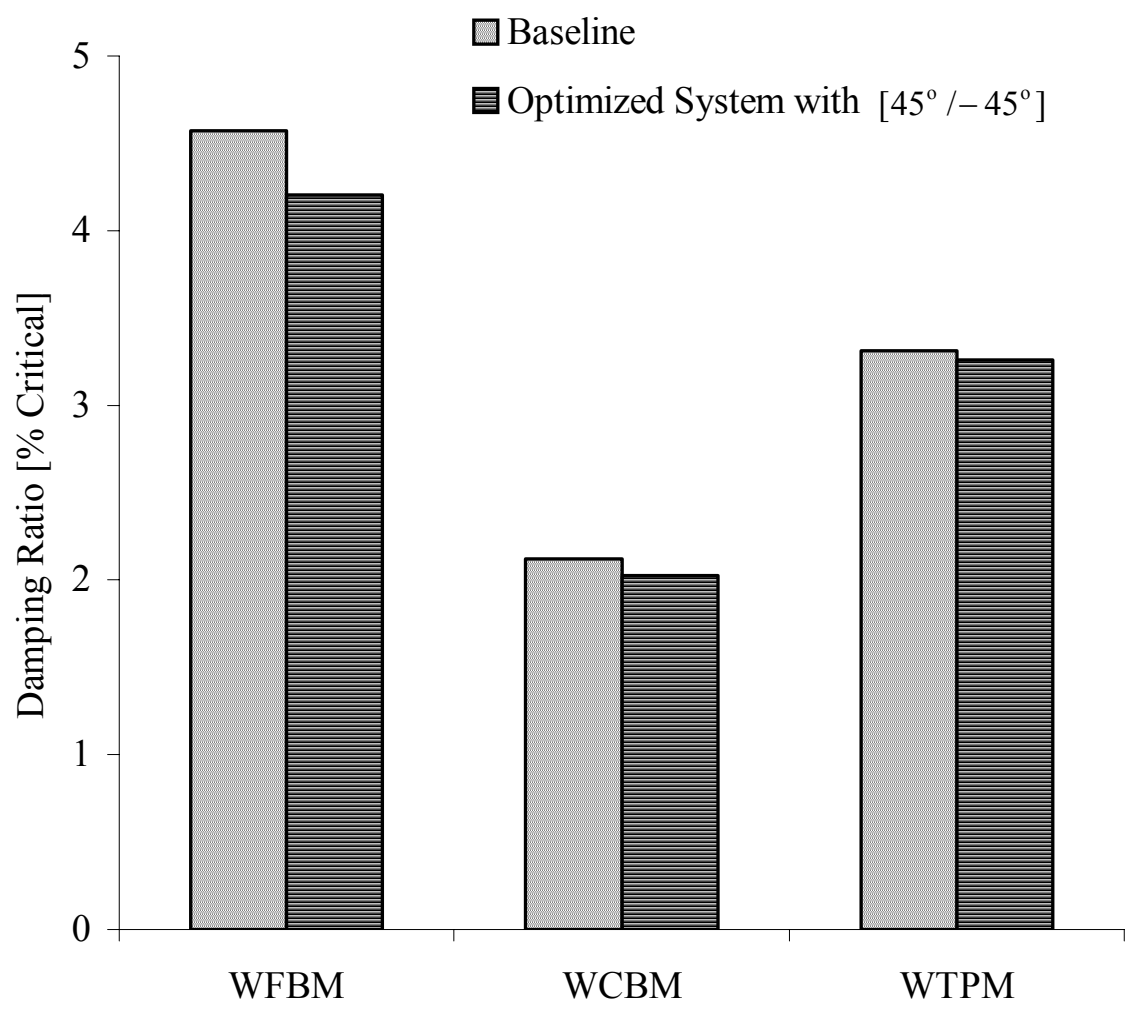

Figure 5-27. Damping Ratio Comparison at 310 Knots 


\section{CHAPTER 6}

\section{Conclusion and Recommendations}

\subsection{Conclusions}

An optimization technique is applied for performance improvement of an XV15 tiltrotor aircraft based on passive blade twist control concept through the use of elastically tailored extension-twist coupled composite rotor blades. The improvement is augmented through the use of the sliding mass concept proposed in this work. The concept makes use of the existing non-structural mass on the rotor blade such as the leading edge balancing mass. This mass is designed to slide with respect to the rotor blade all along the blade span but fixed at the blade tip. This creates an effective way of transferring the centrifugal loads due to this mass to the blade tip and provides an additional flexibility in tailoring the structure without any weight penalty on the system.

The blade structural couplings are determined using a realistic blade cross-section modeled accurately utilizing VABS. The overall aircraft response is evaluated through a detailed multibody dynamics model. Three bladed gimbaled rotor system complete with the swashplate and the pitch links have been modeled in DYMORE. Both DYMORE and VABS have been integrated into an optimization scheme.

Investigations of the baseline design pointed to about 5 to $6 \%$ improvement in hover power requirement and about $1 \%$ improvement in the cruise power requirement when the optimum blade twist distributions are achieved. Based on this investigation the design 
objective is set to attain optimum twist distribution in hover while keeping the baseline twist distribution in cruise.

A two-level design optimization process based on the Simulated Annealing (SA) method has been used for performance improvement. A significant feature of SA based methods is the ability to deal with discrete design space. The present optimization made use of this feature to meet practical manufacturing constraints in terms of discrete ply thickness and angles.

In the lower level optimization the target coupling values required for the optimum rotor blade twist distribution together with the associated sliding mass amount are determined. In this process a simple box-beam model has been used for the cross-sectional analysis. In the upper level optimization a detailed cross-sectional sizing process is performed based on target coupling and stiffness values with constraints on the geometry and failure. The baseline torsional and bending stiffness distributions are sought to be matched to the fullest extend and the system maintains the baseline mass distribution.

Several composite lay-up systems have been considered in the upper level optimization. Due to manufacturability constraints the composite stacking sequences are chosen to be constant over the blade span. The final optimized design resulted in $4.1 \%$ improvement in power requirement in hover at $C_{T}=0.01$. An investigation of the effect of the sliding mass on the system revealed that about $21 \%$ of the improvement is produced by the fact that this mass is allowed to slide with respect to the main blade structure.

To account for the failure mechanisms which are not predicted by the failure criteria used in the optimization, the final optimized system is strengthened by applying a layer of $\left[45^{\circ} /-45^{\circ}\right]$ angle-ply system. An investigation of this new model pointed to about $3.76 \%$ 
improvement in power requirement in hover at $C_{T}=0.01$. The hygrothermal response of this model is checked by a representative ABAQUS model and the hygrothermal effects are found to be minimal.

Aeroelastic stability of the system in cruise mode is checked outside the optimization by DYMORE dynamic simulations. A Prony series fit to the DYMORE data output for a disturbed system showed that the optimized system is stable and the effect of the sliding mass system on the damping characteristics of the aircraft is within $6 \%$.

This research demonstrated an optimization process that integrates a cross-sectional analysis tool together with a multibody dynamics model for performance improvement of a tiltrotor aircraft with tailored composite rotor blades. The data presented in this research showed that it is possible to achieve significant performance improvement in tiltrotor aircraft through the use of extension-twist coupled realistic composite rotor blades. The proposed sliding mass concept provides a significant additional flexibility to tailor the structure without any weight penalty to the system. 


\subsection{Recommendations}

This research points to a number of inquiries worthy of further investigation. These are presented in terms of the following tasks.

- The Sliding Mass concept is studied only numerically in this research. An experimental verification of the effects of the concept on the tension-torsion behavior of tailored composite blades should be investigated.

- The present research involved passive blade twist control only. A consideration of active control together with sliding mass concept might be considered for future studies.

- The multibody dynamics model used did not consider cyclic input to the rotor blades. These inputs might be added to the system for a more realistic model.

- In this research the blade loading used for failure check is determined based on baseline steady state loads. A more detailed model could be constructed by considering the vibratory loads as well.

- The integration of DYMORE into the optimization scheme is a difficult task as not all stiffness matrices provided by the cross-sectional analysis constitute a stable system and hence causes an unstable run in DYMORE. A more detailed integration process is needed where such instances can be avoided to improve the cost of the optimization process.

- The hygrothermal stability of the optimized system is checked by a representative simple constant profile model. An investigation of a non-uniform profile may be done to better understand the response of the system to temperature change. 
- The optimization involved reaching a predetermined optimum target twist distribution as a design goal. However the improvements in figure of merit in hover and axial efficient in forward flight could be set as design objectives as well.

- The rotor speed and rotor geometric parameters such as chord distribution and thickness to chord ratio are kept as the baseline model. The present research could be extended to include these parameters in the design space.

- In the present research the aeroelastic stability investigation of the system utilized Prony series with constant coefficients. A Prony series with periodic coefficients can be used to investigate the response of the system, as some periodicy is included in the system response due to the rotating blades on the wing tip. 


\section{Appendix}

\section{A. Spanwise Variable Layup Case}

The upper level optimization is performed for a case where the constant lay-up constraint is relaxed. The ply angles and the ply thickness values are chosen to be nondiscrete to cover a larger design space. The optimization is performed at five equally spaced locations along the span of the beam for $R>0.5$. The results of the optimization are given in Table A-1 .

Table A-1 Optimum Design Parameters for Spanwise Variable Layup Case

\begin{tabular}{|c|c|c|c|c|c|c|c|}
\hline \multirow[b]{2}{*}{ Span } & \multicolumn{3}{|c|}{ Skin } & \multicolumn{3}{|c|}{ Spar } & \multirow[b]{2}{*}{$\mathrm{r}_{\mathrm{s}} / \mathrm{c}$} \\
\hline & $\theta_{1}[\mathrm{deg}]$ & $\theta_{2}[\mathrm{deg}]$ & $t_{1}[\mathrm{~mm}]$ & $\theta_{1}[\mathrm{deg}]$ & $\theta_{2}[\mathrm{deg}]$ & $t_{2}[\mathrm{~mm}]$ & \\
\hline 0.58 & 28.3 & 28.2 & 3.000 & 45.6 & 88.8 & 5.774 & 0.38 \\
\hline 0.68 & 32.3 & 41.0 & 1.662 & 41.9 & 76.0 & 9.613 & 0.27 \\
\hline 0.79 & 41.7 & 64.5 & 2.507 & 33.9 & 88.1 & 6.422 & 0.20 \\
\hline 0.89 & 20.0 & 19.9 & 0.828 & -65.3 & 47.8 & 11.248 & 0.22 \\
\hline 1.00 & -41.8 & -41.8 & 1.514 & 69.8 & 35.1 & 6.159 & 0.40 \\
\hline
\end{tabular}

It can be seen that the optimization resulted in stacking sequence distributions which are unpractical as the ply angle orientation changes along the span. Unless a new manufacturing technique is developed, manufacturing such a blade may only been possible by producing the blade in sections and joining them along the blade span. However such a blade would entail significant problems associated with the joining process. 
The torsional stiffness and extension-twist coupling distribution associated with this design and their comparison with the target values are depicted in Figure A-1 and Figure A2 , respectively.

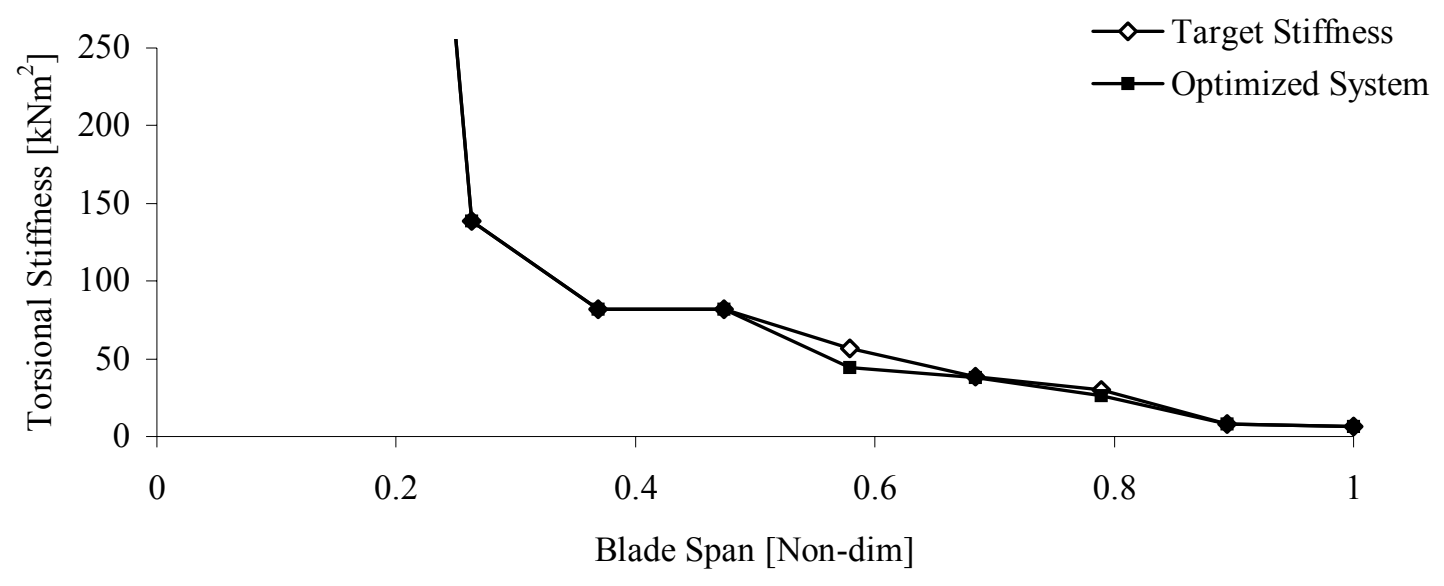

Figure A-1. Torsional Stiffness Distribution

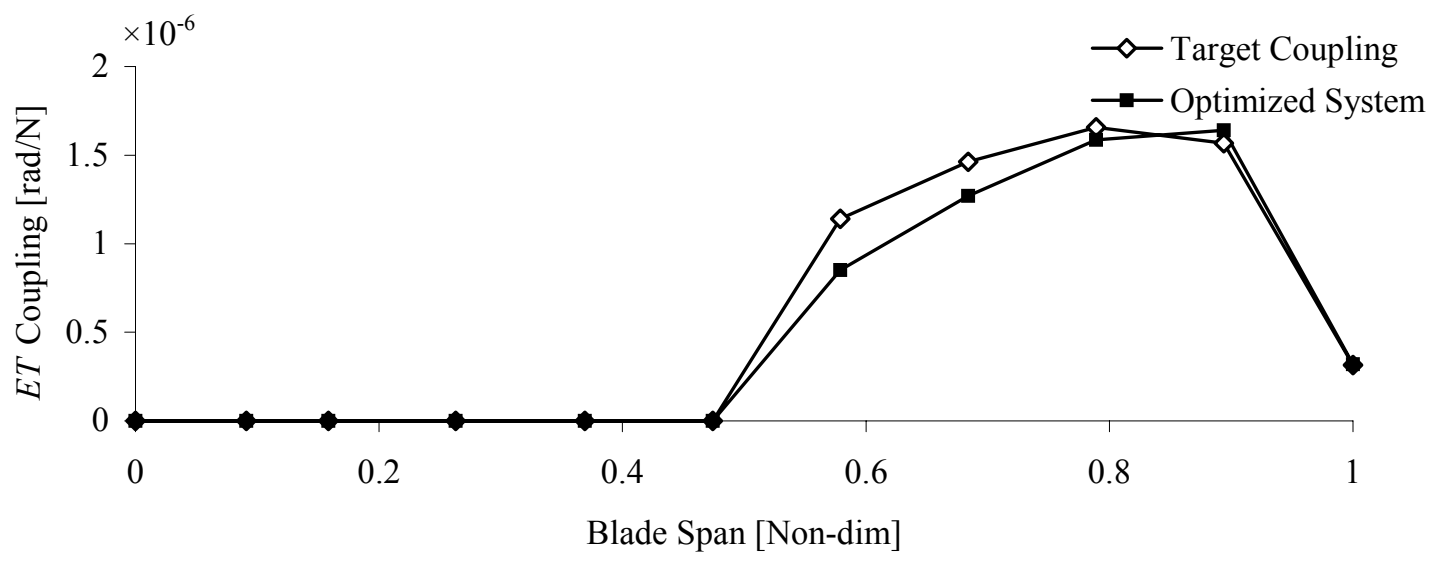

Figure A-2. Extension-Twist Coupling Distribution 
It can be seen that the torsional stiffness is matched within $22 \%$. The real improvement is evident in the target extension-twist coupling values. The coupling is matched to the target value within $2 \%$ for span locations grater than 0.9 and is within $25 \%$ otherwise. A better approximation of the target values are achieved with this case compared to the constant layup model.

The power versus thrust coefficient curve for the system incorporating sliding mass of $0.997 \mathrm{~kg} / \mathrm{m}$, the optimum value provided by the lower level optimizer, points to about $4.19 \%$ improvement in the hover power requirement with respect to the baseline design with a power coefficient of $C_{P}=8.8 \times 10^{-4}$ at $C_{T}=0.01$, as depicted by Figure A-3. This results in about $2.5 \%$ improvement in power saving compared to the constant lay-up case. 


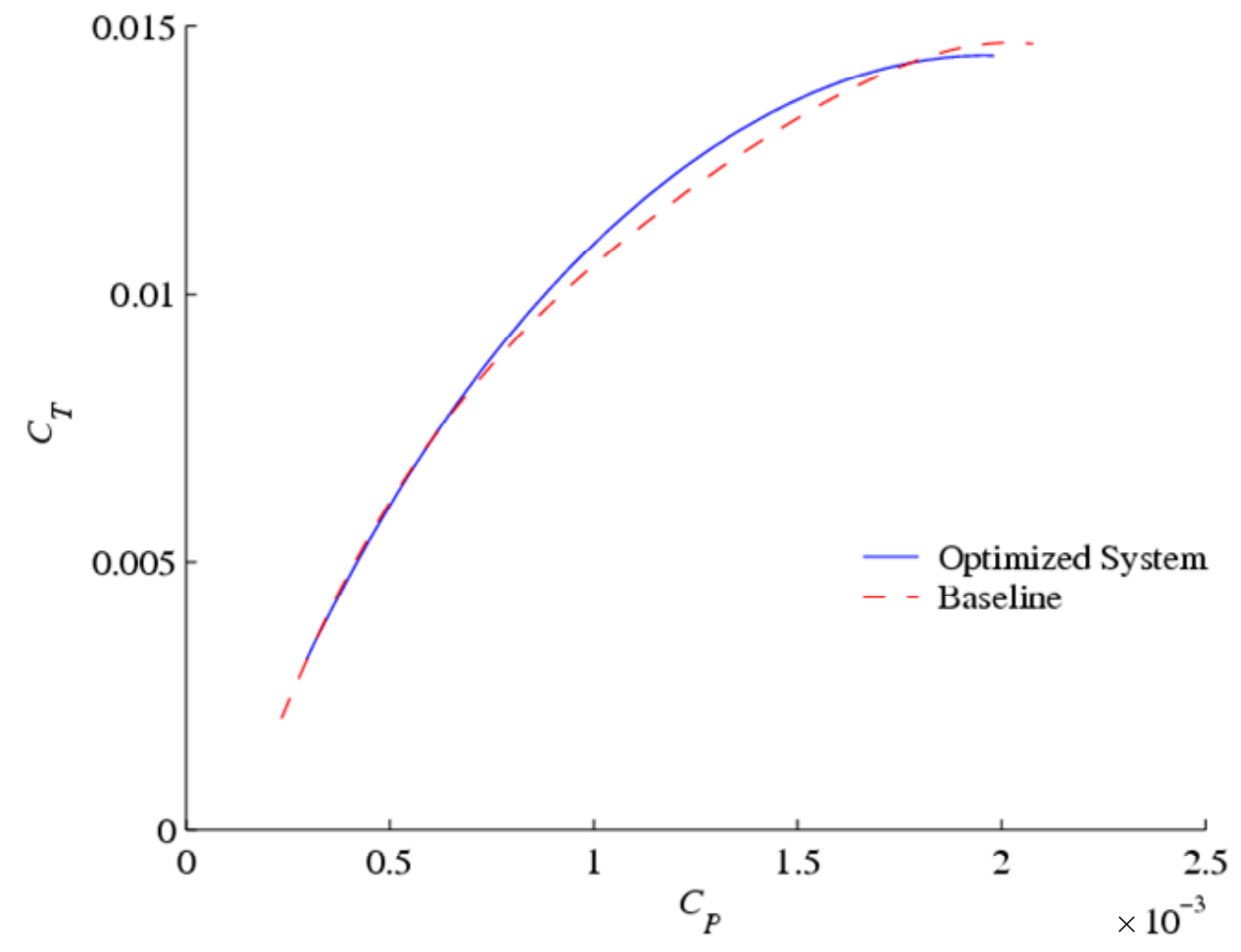

Figure A-3. Power vs. Thrust Coefficient Comparison 


\section{B. Hygrothermally Stable Case}

A hygrothermally stable model with $\left[\theta /-\left(90^{\circ}-\theta\right)_{2} / \theta\right]$ type stacking sequences is investigated for the upper level optimization. This family of stacking sequences was proposed by Winckler [64] and guarantees hygrothermal stability while providing extensiontwist coupling. The optimization is performed such that the skin and the spar are allowed to have different ply angles from the same family of stacking sequences but are kept constant along the blade span for manufacturability considerations. The optimization is performed at five equally spaced locations along the span of the beam for $R>0.5$. The results of the optimization are given in Table B-1.

Table B-1Optimum Design Parameters

\begin{tabular}{|c|c|c|c|c|c|}
\hline \multirow[b]{2}{*}{ Span } & \multicolumn{2}{|c|}{ Skin } & \multicolumn{2}{|c|}{ Spar } & \multirow[b]{2}{*}{$r_{s} / c$} \\
\hline & $\theta_{1}[\mathrm{deg}]$ & $t_{1}[\#$ plies $]$ & $\theta_{2}[\mathrm{deg}]$ & $t_{2}[\#$ plies $]$ & \\
\hline 0.58 & 10 & 16 & 10 & 48 & 0.40 \\
\hline 0.68 & 10 & 16 & 10 & 40 & 0.40 \\
\hline 0.79 & 10 & 16 & 10 & 40 & 0.36 \\
\hline 0.89 & 10 & 12 & 10 & 32 & 0.40 \\
\hline 1.00 & 10 & 4 & 10 & 80 & 0.37 \\
\hline
\end{tabular}

The associated torsional stiffness and extension-twist coupling distribution and their comparison with the target values are provided in Figure B-1 and Figure B-2, respectively. It can bee seen that the target value for the torsional stiffness is not met for up to $50 \%$ of the blade span and the torsional stiffness shows a difference of up to $60 \%$. 


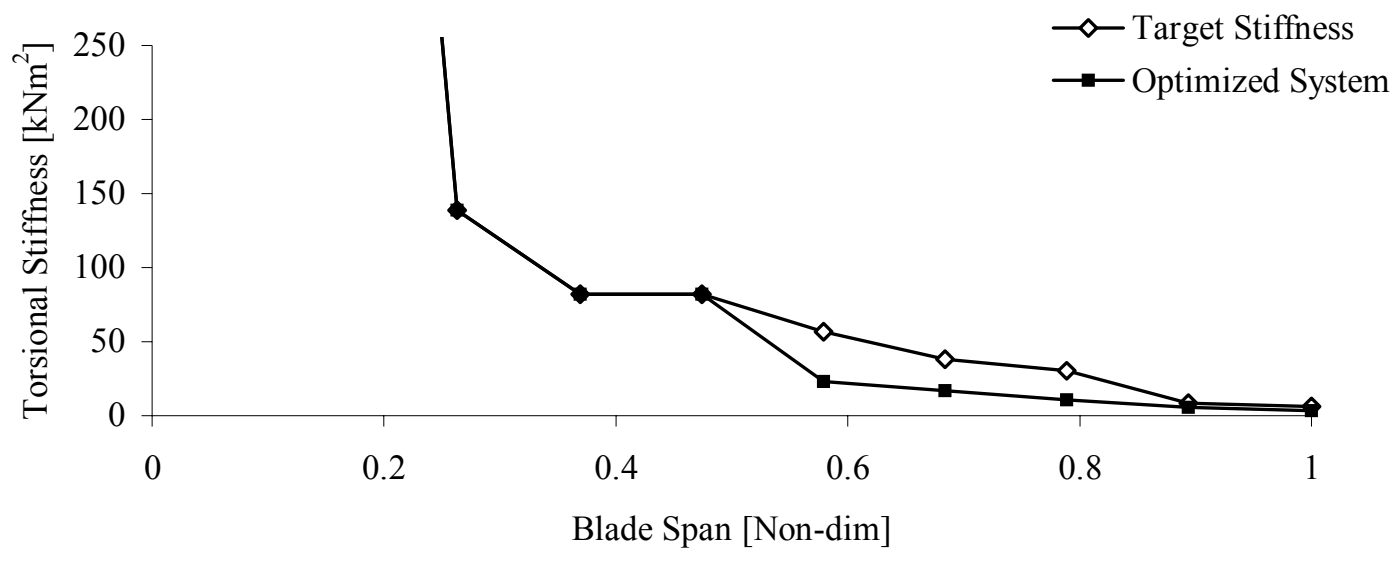

Figure B-1. Torsional Stiffness Distribution

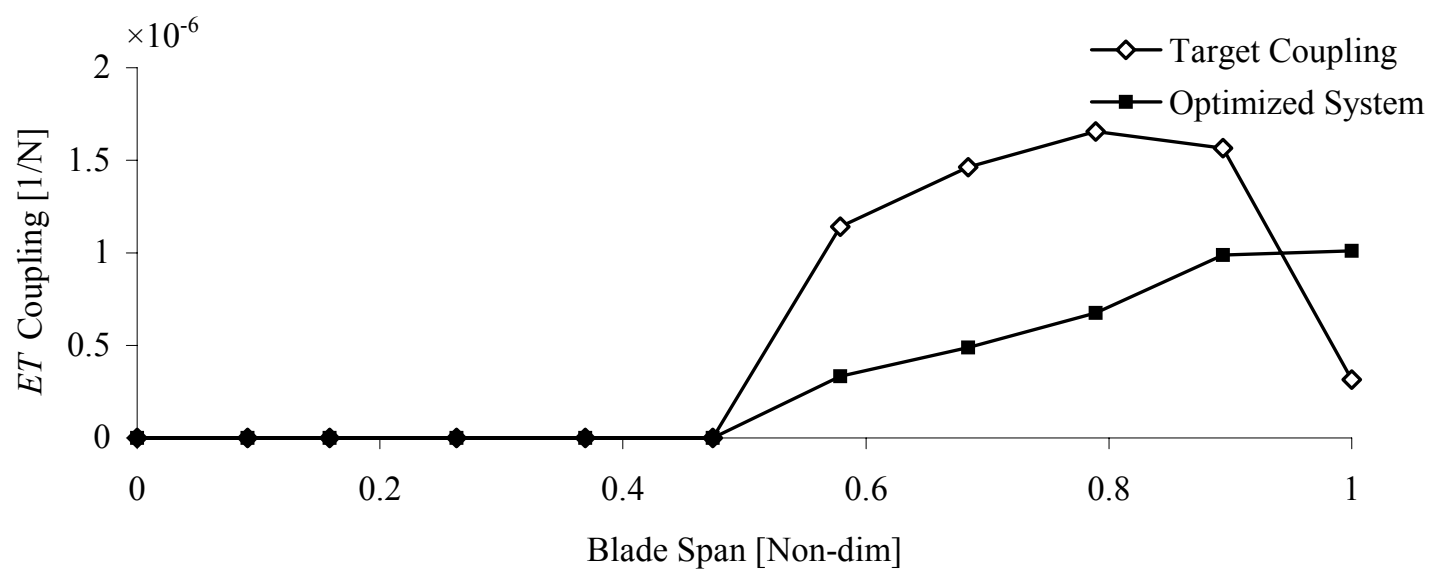

Figure B-2. Extension-Twist Coupling Distribution 
The power versus thrust coefficient curve for the system incorporating the sliding mass of $0.997 \mathrm{~kg} / \mathrm{m}$, the optimum value provided by the lower level optimizer, points to about $2 \%$ reduction in the hover power requirement with respect to the baseline design with a power coefficient of $C_{P}=9.0 \times 10^{-4}$ at $C_{T}=0.01$, as depicted by Figure B-3. However, there is not a distinctive reduction in the power requirement in hover when larger trust coefficient ranges are considered.

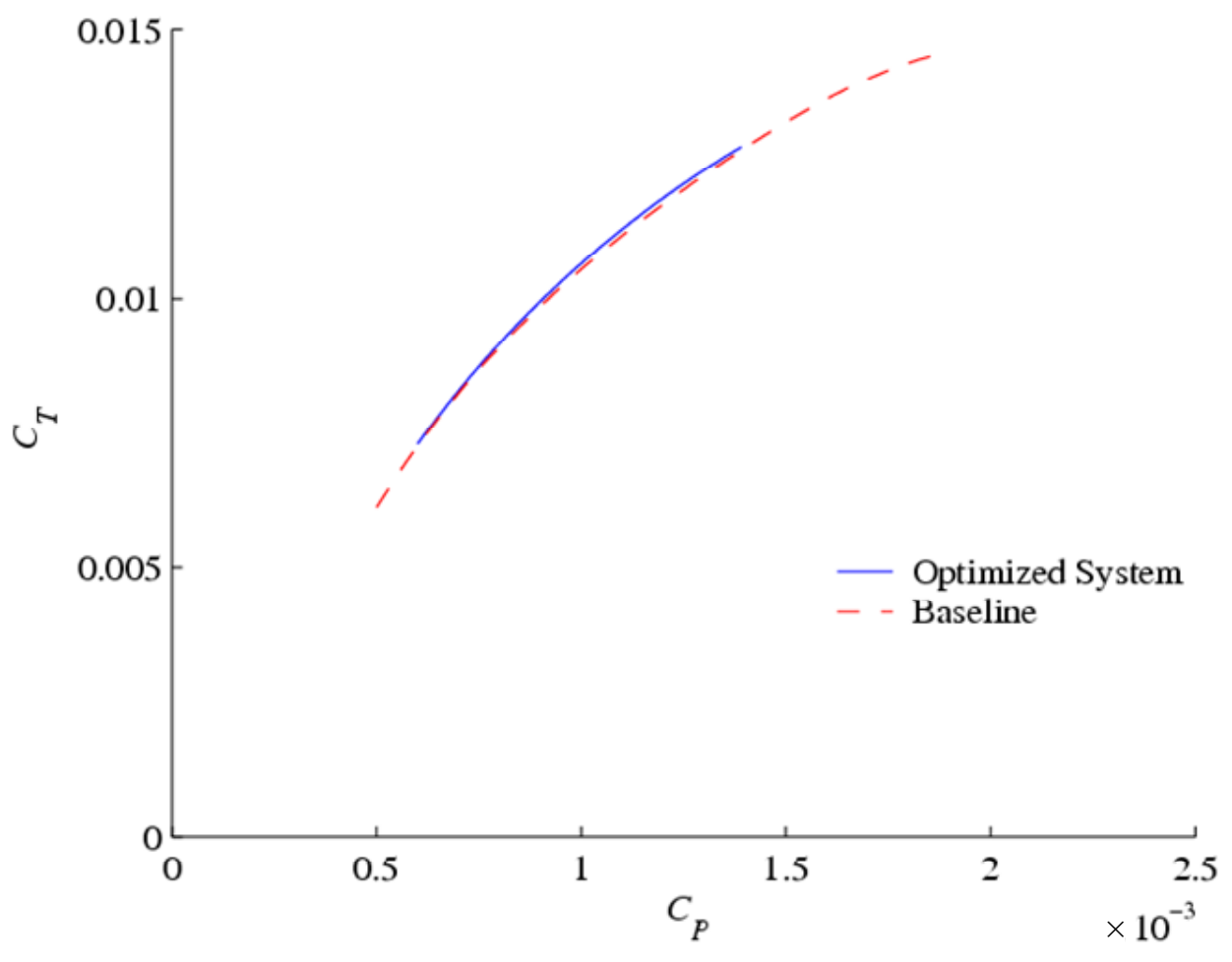

Figure B-3. Power vs. Thrust Coefficient Comparison 


\section{References}

1. Armanios, E.A., Makeev, A., and Hooke, D., "Finite-Displacement Analysis of Laminated Composite Strips with Extension-Twist Coupling", Journal of Aerospace Engineering, Vol. 9, No. 3, 1996, pp. 80-91

2. Barkai, S.M., Rand, O., Peyran, R.J., and Carlson, R.M., "Modeling and Analysis of Tilt-Rotor Aeromechanical Phenomena", Mathematical and Computer Modelling (Oxford), Vol. 27, No. 12, 1998, pp. 17

3. Bartie, K., Alexander, M., Mcveigh, M., La Mon, S., and Bishop, H., "Hover Performance Tests of Baseline Metal and Advanced Technology Blades (ATB) Rotor Systems for the Xv15 Tilt Rotor Aircraft", NASA CR 177436,

4. Bauchau, O.A., "A Beam Theory for Anisotropic Materials", Transactions of the ASME, Journal of Applied Mechanics, Vol. 52, No. 2, 1985, pp. 416-22

5. Bauchau, O.A., Bottasso, C.L., and Nikishkov, Y.G., "Modeling Rotorcraft Dynamics with Finite Element Multibody Procedures", Mathematical and Computer Modelling, Vol. 33, No. 10-11, 2001, pp. 1113-1137

6. Bauchau, O.A., Coffenberry, B.S., and Rehfield, L.W., "Composite Box Beam Analysis: Theory and Experiments", Journal of Reinforced Plastics and Composites, Vol. 6, No. 1, 1987, pp. 25-35

7. Bauchau, O.A., G., L.R., and Bryan, P.S., "An Approach to Ideal Twist Distribution in Tilt Rotor VSTOL Blade Designs", $39^{\text {th }}$ Annual Forum Proceedings - American Helicopter Society, 1983

8. Bauchau, O.A. and Kang, N.K., "Multibody Formulation for Helicopter Structural Dynamic Analysis", Journal of the American Helicopter Society, Vol. 38, No. 2, 1993, pp. 3-14 
9. Bauchau, O.A. and Nikishkov, Y.G., "An Implicit Floquet Analysis for Rotorcraft Stability Evaluation", Journal of the American Helicopter Society, Vol. 46, No. 3, 2001, pp. 200-209

10. Berdichevsky, V., Armanios, A. E., and Badir, A., "Theory of Anisotropic ThinWalled Closed-Cross-Section Beams", Composites Engineering, Vol. 2, No. 5-7, 1992, pp. 411-32

11. Berdichevsky, V. L., "Variational-Asymptotic Method of Construction a Theory of Shell", PMM, Vol. 43, No. 4, 1979, pp. 664-687

12. Borri, M. and Mantegazza, P., "Some Contributions on Structural and Dynamic Modeling of Rotor Blades", L'aerotecnica Missilli E Spazio, Vol. 64, No. 9, 1985, pp. $143-154$

13. Borri, M. and Merlini, T., "A Large Displacement Formulation for Anisotropic Beam Analysis", Meccanica, Vol. 21, No. 1, 1986, pp. 30-7

14. Celi, R., "Recent Applications of Design Optimization to Rotorcraft - a Survey", Annual Forum Proceedings - American Helicopter Society, 1999

15. Cesnik, C.E.S. and Hodges, D.H., "Vabs: A New Concept for Composite Rotor Blade Cross-Sectional Modeling", Journal of the American Helicopter Society, Vol. 42, No. 1, 1997, pp. 27-38

16. Cesnik, C.E.S., Hodges, D.H., and Sutyrin, V.G., "Cross-Sectional Analysis of Composite Beams Including Large Initial Twist and Curvature Effects", AIAA Journal, Vol. 34, No. 9, 1996, pp. 1913-1920

17. Chattopadhyay, A., McCarthy, T.R., and Pagaldipti, N., "Multilevel Decomposition Procedure for Efficient Design Optimization of Helicopter Rotor Blades", AIAA Journal, Vol. 33, No. 2, 1995, pp. 223-230

18. Chattopadhyay, A., McCarthy, T.R., and Rajadas, J.N., "Multidisciplinary Optimization of Tilt Rotor Blades Using Comprehensive Composite Modeling 
Technique", Annual Forum Proceedings - American Helicopter Society, Virginia Beach, VA, 1997, Vol. 1, pp. 809-822

19. Chattopadhyay, A., McCarthy, T.R., and Seeley, C.E., "Decomposition-Based Optimization Procedure for High-Speed Prop-Rotors Using Composite Tailoring", Journal of Aircraft, Vol. 32, No. 5, 1995, pp. 1026-1033

20. Chattopadhyay, A., McCarthy, T.R., and Seeley, C.E., "Multiple Design Point Optimization of High-Speed Proprotors", Journal of Aircraft, Vol. 33, No. 3, 1996, pp. 625-627

21. Dancila, D.S., Kim, I.B., and Armanios, E.A., "Star-Shape Cross-Section ExtensionTwist-Coupled Composite Beams for Rotorcraft Applications", Annual Forum Proceedings - American Helicopter Society, Washington, DC, 1998, Vol. 2, pp. 10441048

22. Fradenburgh, E.A. and Matuska, D.G., "Advancing Tiltrotor State-of-the-Art with Variable Diameter Rotors", Annual Forum Proceedings - American Helicopter Society, Washington, DC, 1992, Vol. 2, pp. 1115-1135

23. Gaffery, T.M., Yen, J.G., and Kvaternik, R.G., "Analysis and Model Tests of the Proprotor Dynamics of a Tilt-Proprotor Vtol Aircraft", Air Force V/STOL Technology and Planning Conference, Las Vegas, NV, 1969

24. Ganguli, R. and Chopra, I., "Aeroelastic Tailoring of Composite Couplings and Blade Geometry of a Helicopter Rotor Using Optimization Methods", Journal of the American Helicopter Society, Vol. 42, No. 3, 1997, pp. 218-228

25. Ghiringhelli, G.L., Masarati, P., Mantegazza, P., and Nixon, M.W., "Multi-Body Analysis of the 1/5 Scale Wind Tunnel Model of the V-22 Tiltrotor", Annual Forum Proceedings - American Helicopter Society, 1999, pp. 1087-1096

26. Giavotto, V., Borri, M., Mantegazza, P., Ghiringhelli, G., Carmaschi, V., Maffioli, G.C., and Mussi, F., "Anisotropic Beam Theory and Applications", Computers and Structures, Vol. 16, No. 1-4, 1983, pp. 403-13 
27. Gregory, R.O., II, Armanios, E.A., and Schleisman, M., "Analysis and Testing of Extension Twist Coupled Composite Using Symmetric Configurations", II Collection of Technical Papers - AIAA/ASME/ASCE/AHS/ASC Structures, Structural Dynamics and Materials Conference, Atlanta, GA, 2000, Vol. 1, pp. 902-907

28. Helf, S., Broman, E., Gatchel, S., and B., C., "Full Scale Hover Test of a 25-Foot Tilt Rotor", BHT Report 300-099-010, NASA CR 114626, 1973

29. Hodges, D.H., "Mixed Variational Formulation Based on Exact Intrinsic Equations for Dynamics of Moving Beams", International Journal of Solids and Structures, Vol. 26, No. 11, 1990, pp. 1253-1273

30. Hodges, D.H., "Review of Composite Rotor Blade Modeling", AIAA Journal, Vol. 28, No. 3, 1990, pp. 561-565

31. Hong, C.-H. and Chopra, I., "Aeroelastic Stability Analysis of a Composite Rotor Blade", Journal of the American Helicopter Society, Vol. 30, No. 2, 1985, pp. 57-67

32. Hooke, D.A. and Armanios, E.A., "Design and Evaluation of Three Methods for Testing Extension-Twist-Coupled Laminates", ASTM Special Technical Publication, Vol. 1274 No. 1996, pp. 340-357

33. Hooke, D.A. and Armanios, E.A., "Rotational Displacement Apparatus with UltraLow Torque and High Thrust Load Capability", US Patent No. 5661247, August 26, 1997 and US Patent No. 5967017, October 19, 1999

34. Ingber, L., "'Adaptive Simulated Annealing (Asa)," Global Optimization C-Code", http://www.ingber.com/\#Asa-Code., [Last accessed on 11/02/2005]

35. Johnson, W., "Development of a Comprehensive Analysis for Rotorcraft .1. Rotor Model and Wake Analysis", Vertica, Vol. 5, No. 2, 1981, pp. 99-129

36. Johnson, W., "Development of a Comprehensive Analysis for Rotorcraft .2. Aircraft Model, Solution Procedure and Applications", Vertica, Vol. 5, No. 3, 1981, pp. 185216 
37. Kim, I. B., Ozbay, S., Dancila, S. D., and Armanios, E. A., "Elastically Tailored Composite Star Configurations with Circular Core Reinforcement for Tilt Rotor Applications", Proceedings of The American Society For Composites $18^{\text {th }}$ Technical Conference, Gainesville, FL, 2003

38. Kvaternik, R.G., "Studies in Tilt Rotor Vtol Aircraft Aerolesticity", Ph.D. Dissertation, Department of Solid Mechanics, Structures And Mechanical Design, Case Western Reserve University, Cleveland, OH, 1973

39. Lake, R.C., Nixon, M.W., Wilbur, M.L., Singleton, J.D., and Mirick, P.H., "A Demonstration of Passive Blade Twist Control Using Extension-Twist Coupling", Collection of Technical Papers - AIAA/ASME/ASCE/AHS/ASC Structures, Structural Dynamics and Materials Conference, Dallas, TX, 1992, pp. 774-781

40. Lake, R.C., Nixon, M.W., Wilbur, M.L., Singleton, J.D., and Mirick, P.H., "Demonstration of an Elastically Coupled Twist Control Concept for Tilt Rotor Blade Application", AIAA Journal, Vol. 32, No. 7, 1994, pp. 1549-1551

41. Makeev, A. and Armanios, E.A., "Elasticity Solution for Torsional Rigidity of Pretwisted Composite Strips", Journal of Engineering Mechanics, Vol. 125, No. 10, 1999, pp. 1147-1153

42. Mansfield, E.H. and Sobey, A.J., "Fiber Composite Helicopter Blade .1. Stiffness Properties .2. Prospects for Aeroelastic Tailoring", Aeronautical Quarterly, Vol. 30, No. May, 1979, pp. 413-449

43. McCarthy, T.R., Chattopadhyay, A., and Zhang, S., "Coupled Rotor/Wing Optimization Procedure for High Speed Tilt-Rotor Aircraft", Annual Forum Proceedings - American Helicopter Society, Fort Worth, TX, 1995, Vol. 2, pp. 924936

44. Nixon, M.W., "Extension-Twist Coupling of Composite Circular Tubes with Application to Tilt Rotor Blade Design", Collection of Technical Papers - 
AIAA/ASME/ASCE/AHS/ASC Structures, Structural Dynamics and Materials Conference, Monterey, CA, 1987, pp. 295-303

45. Nixon, M.W., "Aeroelastic Response and Stability of Tiltrotors with ElasticallyCoupled Composite Rotor Blades", Ph.D. Dissertation, University of Maryland, 1993

46. Nixon, M.W., Piatak, D.J., Corso, L.M., and Popelka, D.A., "Aeroelastic Tailoring for Stability Augmentation and Performance Enhancements of Tiltrotor Aircraft", Journal of the American Helicopter Society, Vol. 45, No. 4, 2000, pp. 270-279

47. Nixon, W.M., "Improvements to Tilt Rotor Performance through Passive Blade Twist Control", NASA TM-100583, 1988

48. Ozbay, S., Tawfik, S., Tan, X., and Armanios, E. A., "Optimum Stacking Sequences for Extension-Twist Coupled Strips under Mechanical Loading", ASC/ASTM-D30 Joint $19^{\text {th }}$ Annual Technical Conference, Atlanta, GA, 2004

49. Peters, D.A. and He, C.J., "Correlation of Measured Induced Velocities with a FiniteState Wake Model", Journal of the American Helicopter Society, Vol. 36, No. 3 , 1991, pp. 59-70

50. Peters, D.A. and He, C.J., "Finite State Induced Flow Models Part Ii: ThreeDimensional Rotor Disk", Journal of Aircraft, Vol. 32, No. 2, 1995, pp. 323-333

51. Popelka, D., Sheffler, M., and Bilger, J., "Correlation of Stability Test Results and Analysis for the 1/5 Scale V-22 Aeroelastic Model", Annual Forum Proceedings American Helicopter Society, Fort Worth, TX, 1985, Vol. 1, pp. 17-31

52. Prahlad, H. and Chopra, I., "Design of a Variable Twist Tiltrotor Blade Using Shape Memory Alloy (Sma) Actuators", Proceedings of the SPIE - The International Society for Optical Engineering, Vol. 4327, 2001, pp. 46-59

53. Quackenbush, T.R., Wachspress, D.A., and Kaufman, A.E., "Optimization of Rotor Performance in Hover Using a Free Wake Analysis", Journal of Aircraft, Vol. 28, No. 3, 1991, pp. 200-207 
54. Rehfield, L.W., "Design Methodology for Composite Rotor Blades", AFWAL-TR-853094, Vol. No. 1985, pp. (V(A)-1) - (V(A)-15)

55. Sakata, S., "Asamin", http://www.econ.ubc.ca/ssakata/public_html/software/, [Last accessed on 11/28/2005]

56. Shang, X., "Aeroelastic Stability of Composite Hingless Rotors with Finite-State Unsteady Aerodynamics", Ph.D. Dissertation, Georgia Institute of Technology, Atlanta,GA, 1995

57. Smith, E.C. and Chopra, I., "Formulation and Evaluation of an Analytical Model for Composite Box-Beams", Collection of Technical Papers - AIAA/ASME/ASCE/AHS Structures, Structural Dynamics \& Materials Conference, Long Beach, CA, 1990, pp. $759-782$

58. Sobieszczanski-Sobieski, J. and Haftka, R.T., "Multidisciplinary Aerospace Design Optimization: Survey of Recent Developments", Structural Optimization, Vol. 14, No. 1, 1997, pp. 1-23

59. Soykasap, O., "Aeroelastic Optimization of a Composite Tiltrotor", Ph.D. Dissertation, Georgia Institute of Technology, Atlanta, GA, 1999

60. Soykasap, O., "Inverse Method in Tilt-Rotor Optimization", Aerospace Science and Technology, Vol. 5, No. 7, 2001, pp. 437-444

61. Soykasap, O. and Hodges, D.H., "Performance Enhancement of a Composite TiltRotor Using Aeroelastic Tailoring", Journal of Aircraft, Vol. 37, No. 5, 2000, pp. $850-858$

62. Volovoi, V.V. and Hodges, D.H., "Theory of Anisotropic Thin-Walled Beams", Transactions of the ASME-Journal of Applied Mechanics, Vol. 67, No. 3, 2000, pp. 453-459 
63. Walsh, J.L., Young, K.C., Pritchard, J.I., Adelman, H.M., and Mantay, W.R., "Integrated Aerodynamic/Dynamic/Structural Optimization of Helicopter Rotor Blades Using Multilevel Decomposition", NASA Technical Report 3465, 1995

64. Winckler, S.J., "Hygrothermally Curvature Stable Laminates with Tension-Torsion Coupling", Journal of the American Helicopter Society, Vol. 30, No. 3, 1985, pp. 5658

65. Worndle, R., "Calculation of the Cross-Section Properties and the Shear Stresses of Composite Rotor Blades", Vertica, Vol. 6, No. 2, 1982, pp. 111-129

66. Yu, W., http://www.mae.usu.edu/faculty/wenbin/ht_docs/vabs.html, [Last accessed on $11 / 28 / 2005$ ]

67. Yu, W., Volovoi, V.V., Hodges, D.H., and Hong, X., "Validation of the Variational Asymptotic Beam Sectional Analysis", AIAA Journal, Vol. 40, No. 10, 2002, pp. 2105-2112 